\author{
Universidade de São Paulo \\ Instituto de Astronomia, Geofísica e Ciências Atmosféricas \\ Departamento de Astronomia
}

\title{
Accretion discs, jets, and black hole spins: a study of blazars
}

Gustavo Rodrigues Romano Soares

São Paulo

2020 

Gustavo Rodrigues Romano Soares

\section{Accretion discs, jets, and black hole spins: a study of blazars}

Tese apresentada ao Departamento de Astronomia do Instituto de Astronomia, Geofísica e Ciências Atmosféricas da Universidade de São Paulo como requisito parcial para a obtenção do título de Doutor em Ciências.

Área de Concentração: Astronomia Orientador(a): Prof. Dr. Rodrigo Nemmen da Silva

Versão Corrigida. O original encontrase disponível na Unidade.

São Paulo, 2020 

To mom and dad. 



\section{Abstract}

Blazars are among the most powerful astrophysical objects in the universe. Their multiwavelength emission displays traces of non-thermal radiation whose origin is not yet fully understood, and it is dominated by the presence of a relativistic jet. Blazar emission is characterized by high-variability across different wavelenghts, which is associated with a spinning black hole and relativistic effects in the jet. Using blazars as a laboratory, in this thesis we set out to answer a few fundamental questions, such as where and how does the non-thermal emission in blazars originate?, how robust are theoretical models in explaining the efficiency of jet formation?, and can these models accurately predict the spins of the black holes associated with these jets? To answer these questions, we employ two different methods: $\gamma$-ray observations and general relativistic magnetohydrodynamic (GRMHD) simulations. In the first study, we used the luminosities of a class of blazars to calculate the jet efficiency, and we estimated the black holes spins. We found a mean spin of $a_{*}=0.84_{-0.25}^{+0.11}$, with a lower limit estimated at $a_{*}^{\text {lower }}=0.59$. These results show compatibility with cosmological merger-driven evolution of SMBHs which support rapidly rotating black holes. Moreover, we found a correlation between the black hole mass and the $\gamma$-ray luminosity $L_{\gamma}$. In the second study, we used GRMHD simulations and applied an algorithm to identify the regions in which non-thermal emission must occur. We ran simulations with different initial conditions, varying the magnetic field topology and black hole spin, and we found these regions in all simulations. In particular, we found that this also occurs in the jet for some simulations, thus suggesting 
that it is possible to apply radiative transfer to simulation data in order to model nonthermal emission in different astrophysical contexts. 


\section{Resumo}

Blazares são alguns dos objetos astrofísicos mais poderosos no universo. Sua emissão ao longo de vários comprimentos de onda apresenta traços de radiação não-térmica cuja origem ainda não é inteiramente compreendida, e ela é dominada pela presença de um jato relativístico. A emissão em blazares é caracterizada por alta variabilidade em vários comprimentos de onda, a qual é associada a um buraco negro em rotação e efeitos relativísticos no jato. Usando blazares como laboratórios, nesta tese nós visamos responder algumas questões fundamentais, tais como onde e como a emissão não-térmica em blazares se origina?, o quão robustos são os modelos teóricos para explicar a eficiência da formação de jatos?, e tais modelos podem prever com precisão os spins dos buracos negros associados a esses jatos? Para responder a essas questões, nós empregamos dois métodos diferentes: observações em raios- $\gamma$ e simulações magnetohidrodinâmicas em relatividade geral (GRMHD). No primeiro estudo, utilizamos a luminosidade de uma classe de blazares para calcular a eficiência dos jatos e estimamos os spins dos buracos negros. Nós encontramos um valor médio de $a_{*}=0.84_{-0.25}^{+0.11}$ para o spin, com um limite inferior estimado em $a_{*}^{\text {lower }}=0.59$. Esses resultados mostram compatibilidade com a evolução de buracos negros supermassivos por meio de fusões, originando buracos negros com alta rotação. Além disso, encontramos uma correlação entre a massa dos buracos negros e a luminosidade em raios- $\gamma, L_{\gamma}$. No segundo estudo, usamos simulações GRMHD e aplicamos um algoritmo para identificar as regiões em que a emissão não-térmica deve ocorrer. Fizemos simulações com diferentes condições iniciais, variando a topologia 
do campo magnético e o spin do buraco negro, e encontramos tais regiões em todas as simulações. Em particular, encontramos que isso também ocorre nos jatos para algumas simulações, sugerindo que é possível aplicar transferência radiativa nos dados de simulações para modelar a emissão não-térmica em diferentes contextos astrofísicos. 


\section{Acknowledgements}

I'm not sure if having taken an Introduction to Astronomy course during my first year as an undergrad counts as having some experience in Astronomy, but this is all Astronomy I had when I decided to switch after a Masters in Theoretical Physics. As it turns out, I ended up doing Theoretical Astrophysics, and the whole transition was smoother than I had anticipated.

I thank my advisor Rodrigo Nemmen, for trusting me, for his patience, help, guidance, and for all advice given on the many aspects of doing research, and academia in general. I also thank Prof. Alexander Tchekhovskoy for hosting me during my visit to Northwestern University, and for sharing his vast knowledge on black holes, accretion, jets and especially simulations.

Being new to Astronomy meant I had to do lots of learning in little time. I thank professors Eduardo Cypriano, Elisabete Dal Pino, Jorge Horvath, Antônio Mario Magalhães, Jorge Meléndez, Claudia Mendes de Oliveira, and Roderik Overzier for their courses, lectures and/or conversations and discussions over the years, which made it far less complicated to adapt to a new field. I also thank Prof. Elisabete Dal Pino for her comments throughout the years as a member of my thesis committee, for telling me to focus when I had to focus, and for reminding me to pay attention to the units. It's not that I don't like cgs, it's just that life was easier when everything was equal to 1.

I thank Profs. Tchekhovskoy, Dal Pino, Overzier, Zulema Abraham and Bruno Carneiro da Cunha for their valuable comments and suggestions as part of my thesis evaluation committee.

I also thank Silvia Rossi for being patient to answer countless questions and for helping me navigate through the multidimensional bureaucratic mazes of IAG-USP. Speaking of IAG-USP, I can't thank enough the staff at the Institute for their invaluable help in dealing with daily issues - from technical and computational to bureaucratic 
and academic: Carlos Paladini, Ulisses Castello, Marco Antonio dos Santos, Lígia, Michel, and Thiago.

I'm very grateful to Katherine Lamb and Peter Anglada at Northwestern University, who handled all the necessary paperwork related to my visit to CIERA and showed me around the department. Big shout out to John Ruckdaeschel for going out of his way to help me find a place to stay in Evanston, and Ying Qin, who shared the visitor's office at CIERA for most of the duration of our stay.

To André, Artur, Catarina, Fabio, Geeh, Ivan, Malu Dantas, Malu Gubolin, Mirian, Rafael, Raniere, Roberta. Some started as friends and then became colleagues, while for others it was the other way around. Anyway, I'm glad these lines are blurred, and I thank you for all the laughs and conversations about virtually everything. I also thank many of you, as well as my high school friends (Cássio, Gildo, Luana, Pão, Perereca, Tio Zé) for being there when my I had difficult health issues in the family back in 2016, and a year later when it was my turn to scare everybody.

To Demônios de Maxwell Rugby, for all the practice sessions, matches, championships, post-match sessions, and the overall fun which is being part of this team.

To Fran, the biggest and best surprise that happened to me in Chicago. Thank you for being so supportive, for caring, for listening, for knowing when to be there and also for knowing when not to be there.

To my mom and dad, for teaching me pretty much everything I know, and for all the selfless sacrifices. To aunt and uncle for their support, even if from a distance.

This work was funded by CAPES. 


\section{Contents}

$\begin{array}{ll}\text { Abstract } & \text { iii }\end{array}$

Resumo $\quad$ v

Acknowledgements vii

List of Figures $\quad$ xvii

List of Tables $\quad$ xx

List of Abbreviations $\quad$ xxi

1 The Stage 1

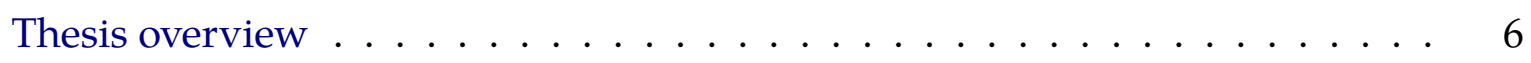

2 Black Holes in Astrophysics $\quad 7$

2.1 Introduction ...................... 7

2.2 Rotating black holes . . . . . . . . . . . . . . . 8

2.2.1 Boyer-Lindquist coordinates . . . . . . . . . . 8

2.2.2 Kerr-Schild coordinates . . . . . . . . . . . . . . 10

2.2.3 Ergosphere, frame-dragging, and energy extraction . . . . . . . 10

2.3 Accretion discs and jets . . . . . . . . . . . . . . . . . 13

2.3 .1 Thin discs . . . . . . . . . . . . . . . . 15

2.3.2 A thin disc solution . . . . . . . . . . . . . 16 
2.3.3 Advection-dominated accretion flows (ADAFs) . . . . . . . . . 18

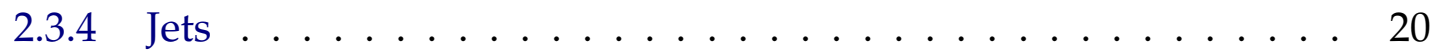

2.4 Active Galactic Nuclei . . . . . . . . . . . . . . . . . . . . . . . . 22

2.4 .1 Radio galaxies . . . . . . . . . . . . . . . . . 25

2.4 .2 Radio-loud quasars . . . . . . . . . . . . . . 25

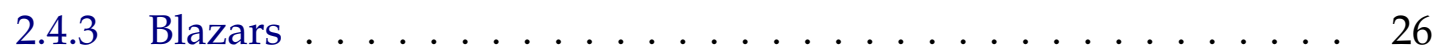

3 Numerical Methods $\quad 31$

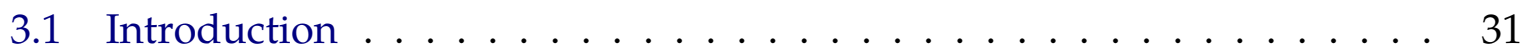

3.2 The equations of GRMHD . . . . . . . . . . . . . . . 33

3.3 Numerical outline . . . . . . . . . . . . . . . . . . . 36

3.4 The MHD stress-energy tensor in GR . . . . . . . . . . . . . . . 38

3.4.1 Covariant formulation of electromagnetism . . . . . . . . . . 38

3.4.2 The equations of GRMHD: derivation . . . . . . . . . . . . . 40

3.5 Numerical methods . . . . . . . . . . . . . . . . . . . . . . . . 44

3.5.1 Riemann Problem . . . . . . . . . . . . . . . . . . 44

3.5.2 Integral Forms of Conservation Laws . . . . . . . . . . . . . 45

3.5.3 Discretization and Conservative Methods . . . . . . . . . . . . 45

3.5.4 The HLL Riemann Solver . . . . . . . . . . . . . . . . . . . . 49

4 Jet efficiencies and black hole spins in jetted quasars 53

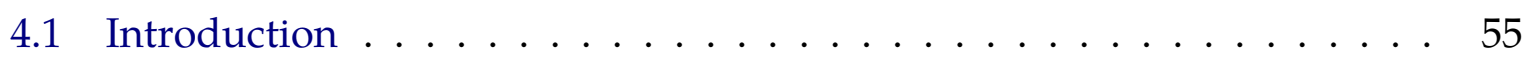

4.2 The Fermi Gamma-ray Space Telescope . . . . . . . . . . . . . . . . 59

4.3 Data selection .......................... 61

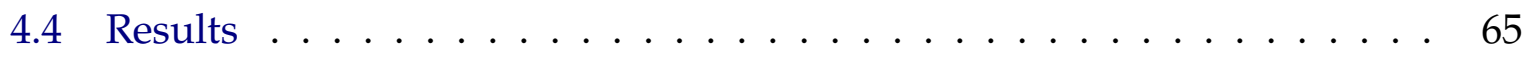

4.4 .1 Black hole spins . . . . . . . . . . . . . . . . 69

4.5 Discussion ................................ 74

4.5.1 Relation with SMBH and galaxy evolution . . . . . . . . 76 
4.5 .2 The extremes of blazar jets . . . . . . . . . . . . . 77

4.5 .3 Caveats ............................ 78

4.6 Conclusions . . . . . . . . . . . . . . . . . . 79

4.7 Data . . . . . . . . . . . . . . . . . . 80

5 Current Sheets in Jets $\quad 85$

5.1 Introduction $\ldots \ldots \ldots \ldots \ldots$

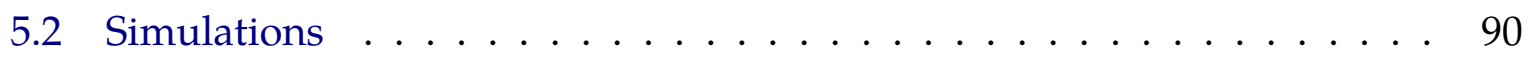

5.3 Comparison of simulations $\ldots \ldots \ldots \ldots$

5.4 Identifying current sheets $\ldots \ldots \ldots \ldots \ldots$

5.5 Ongoing work and next steps . . . . . . . . . . . . . . . 100

6 Conclusions and future directions 107

$\begin{array}{ll}\text { Bibliography } & 134\end{array}$ 


\section{List of Figures}

1.1 The quasar 3C 273 observed in the X-ray by the Chandra X-Ray Observatory, with the jet clearly visible in the center/bottom right. Credit: Herman Marshall/NASA Public Domain - see also Marshall et al. (2001).

1.2 Radio image by the Event Horizon Telescope showing the shadow of the black hole at the core of the galaxy M87 and the surrounding accretion disc. Credit: Event Horizon Telescope Collaboration. . . . . . . . . . .

2.1 Spectral energy distribution of Sgr A* according to a hot ADAF model. Circles with error bars are radio and milimiter wavelengths, circles with arrows are IR upper limits and bowties correspond to X-ray data. The dot-dashed line corresponds to synchrotron emission and the Compton humps from thermal electrons, the short-dashed line shows synchrotron emission from nonthermal electrons, and the long-dashed line indicates bremsstrahlung from electrons close to the Bondi radius. The ADAF model spectrum is the thick solid line, which is a sum of the three components mentioned above, and the dotted line shows the total synchrotron and inverse Compton emission. Source: (Yuan and Narayan

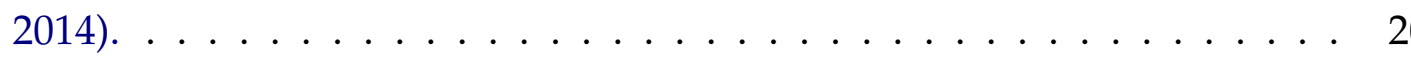


2.2 Radio images of a Fanaroff-Riley type I (left) and Fanaroff-Riley type II (right) galaxy. The image of 3C 296 shows very clearly the higher brightness towards the central part of the galaxy, where it appears redder. The peak brightness in 3C 98 is seen at the end of the lobes. The colored images were taken from http://www . jb.man.ac.uk/atlas/. The image on the left appears on Leahy and Perley (1991), while the image on the right is unpublished and credited to A. H. Bridle. . . . . . . . . . . .

2.3 Spectral energy distribution of blazar Mrk 421, taken from Baloković et al. (2016). The solid blue line shows a one-zone synchrotron self-Compton (SSC) model based on Böttcher et al. (2013), while the dashed red lines show SED models with time-averaged electron distribution. The radio data should be considered as upper limits for the SSC model as Mrk 421 is not well-resolved for single-dish radio instruments, so these points include radio emission from scales larger than the jet itself. . . . . . . .

2.4 The blazar sequence across the electromagnetic spectrum, taken from Ghisellini et al. (2017), adapted from Fossati et al. (1998). From top to bottom: FSRQ, low-energy peaked BL Lac, intermediate-energy peaked BL Lac, high-energy peaked BL Lac, and TeV BL Lac. . . . . . . . . . . 28

2.5 Optical spectrum for a FSRQ, taken from Perlman et al. (1998). . . . . . . 29

2.6 Optical spectrum for a BL Lac, taken from Perlman et al. (1998). . . . . . 29

4.1 The $\gamma$-ray sky: energies greater than $1 \mathrm{GeV}$ as detected on the first five years of data from Fermi-LAT. Source: NASA/DOE/Fermi LAT Collab-

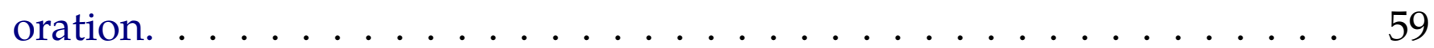

4.2 Redshift distribution of the 154 FSRQs in our sample. . . . . . . . . . . 63

4.3 Distribution of the black hole masses $M$ in our sample. . . . . . . . . . . 64

4.4 Distribution of the gamma-ray luminosities in our sample. . . . . . . . . 64 
4.5 Black hole mass estimates collected from G15 plotted against the FermiLAT gamma-ray flux. . . . . . . . . . . . . . . . . . . . 65

4.6 Black hole mass plotted against the Fermi-LAT gamma-ray luminosity. The uncertainties in $L_{\gamma}$ are too small and barely visible, while the uncertainties in the black hole mass are much larger. The solid blue line corresponds to our best-fit line. . . . . . . . . . . . . . . 66

4.7 Distribution of the jet production efficiencies for the FSRQs in our sample assuming that the SMBHs are accreting at $10 \%$ of the Eddington rate. . .

4.8 Jet production efficiencies as a function of $\mathrm{BH}$ mass. See caveats mentioned in Section $4.4 \ldots \ldots \ldots \ldots \ldots$

4.9 Lower limits on the jet efficiencies of the FSRQs. The horizontal line corresponds to the limiting efficiency assuming a maximum allowed spin and a disc thickness $h=0.13$ in Equation (4.4.4). . . . . . . . . 71

4.10 Distribution of spins. . . . . . . . . . . . . . . 72

4.11 Spin estimates for the 118 FSRQs which have efficiencies consistent with the model we adopted. . . . . . . . . . . . . . . . . . 72

4.12 Spin lower limits as a function of the mass. . . . . . . . . . 73

4.13 Distribution of spin lower limits. . . . . . . . . . . . . . . . 73

4.14 Jet powers versus the accretion power $\dot{M} c^{2}$. The Solid blue line indicates the best-fit relation of G14 and the dashed red line corresponds to $P=$ $\dot{M} c^{2}$. The points are systematically below the G14 due to the method that we used to constrain $P \ldots \ldots \ldots \ldots$. . . . . . . . . . . . . .

5.1 The top panel shows the particle density in a reconnection layer from a PIC simulation. The other three panels are zoomed-in plots of the region between $x=0$ and $x=2500$. They show, from top to bottom, the particle density, magnetic energy and mean kinetic energy per particle. Taken from Sironi and Spitkovsky (2014). . . . . . . . . . . . . . . . . . 88 
5.2 Initial configuration of the magnetic field topology (MAD) in simulation $2 \mathrm{dm} 9$, zoomed in (left) and out (right). . . . . . . . . . . . . . . 91

5.3 Initial configuration of the magnetic field topology (SANE) in simulation $2 \mathrm{ds} 9$.

5.4 Toroidal component of the lab-frame magnetic field, $B_{\phi}$, in four different simulations, all at the same time $t=8000 r_{\mathrm{g}} / \mathrm{c}$. The torus is smaller for $a_{*}=0.1$ due to the Fishbone-Moncrief prescription. $\ldots \ldots \ldots$

5.5 Toroidal component of the magnetic field $\left(B_{\phi}\right)$ in the lab frame in simulation 2ds9. The dash-dotted line corresponds to $b^{2} / \rho=1 \ldots$. . . . . 94

5.6 Gas reaches black hole at around $t=2600 r_{\mathrm{g}} / c$ in simulation $2 \mathrm{dm} 9 . \quad \ldots \quad 95$

5.7 Toroidal component of the magnetic field $\left(B_{\phi}\right)$ in the lab frame in simulation $2 \mathrm{dm} 9$. The dash-dotted line corresponds to $b^{2} / \rho=1 \ldots \ldots 6$

5.12 Magnetization $\sigma$ at four different radii (in $r_{\mathrm{g}}$ ): 5, 10, 15, 20. Saturation value for sigma is at around $\sigma=40-50 \ldots \ldots \ldots 10 \ldots$

5.8 Left: gas density. Right: toroidal component of the lab-frame magnetic field, $B_{\phi}$, in simulation $2 \mathrm{dm} 9$. The gas is sometimes prevented from reaching the black hole due to the magnetic fields accumulating at its vicinity. This behavior occurred multiple times throughout the simulation.103

5.9 Left panel: mock current sheets, where we predetermined the values of the current in each cell. The white cells corresponds to the equivalent of $J_{\text {peak }}$, the maximum value of the current in the snapshot. The colored cells correspond to the current sheets. Right panel: current sheets found by the algorithm corresponds to the predetermined sheet, suggesting the algorithm correctly identifies the current sheets. White and colored cells same as in left panel. The $\mathrm{x}$ and $\mathrm{y}$ axes simply display the spatial position and are of no physical interest. . . . . . . . . . . . . . 104 
5.10 Simulation $2 \mathrm{ds} 9$. Toroidal magnetic field and current sheets in the accretion disc and near the jet basis (circled in red), as identified by the algorithm. Sheets are identified using the magnetization in the toroidal direction. . . . . . . . . . . . . . . . . . 105

5.11 Simulation $2 \mathrm{dm} 9$. Current sheets in the accretion disc and near the jet basis, as identified by the algorithm. Sheets are identified using the magnetization in the toroidal direction. . . . . . . . . . . . 106 


\section{List of Tables}

2.1 Classification of AGN according to their optical properties. Adapted from Blandford, Meier, and Readhead (2019). . . . . . . . . . . . . . . 24

2.2 Radio-loud AGN classified according to their radio properties and bolometric luminosities, as seen in Blandford, Meier, and Readhead (2019). . 24

4.1 Results of partial correlation analysis. Columns (1)-(2): Quantities ( $\mathrm{Z}$ is $\log _{10} d_{\mathrm{L}}$ in both cases). Column (3): Subsample; Column (4): Number of sources; Columns (5)-(7): results of partial correlation analysis; $\tau$ is the partial Kendall's correlation coefficient; $\sigma$ is the square root of the calculated variance; $P_{\text {null }}$ is the probability for accepting the null hypothesis that there is no correlation between $\mathrm{X}$ and $\mathrm{Y}$; Column (7) gives the associated significance in standard deviations with which the null hypothesis is rejected. . . . . . . . . . . .

4.2 The four high-efficiency blazars that cannot be explained by the simulationbased model considered. . . . . . . . . . . . . . . . . . . . . . 74

4.3 Data on the objects used in this work. Spins denoted by one asterisk $\left(^{*}\right)$ could not be obtained using the jet efficiency $\eta$ as input to the simulationbased model in Equation 4.4.4, while lower limit spins denoted by two asterisks $\left({ }^{* *}\right)$ could not be obtained using the same equation for the lower limit jet efficiency $\eta_{\min }$ as input. The uncertainties in mass and jet power are $0.5 \operatorname{dex} \ldots \ldots \ldots \ldots \ldots \ldots \ldots \ldots$ 
5.1 Simulations considered and their parameters. The spatial resolution $\left(N_{r} \times N_{\theta} \times N_{\phi}\right)$ is $288 \times 192 \times 1 \ldots \ldots \ldots \ldots$ 


\section{List of Abbreviations}

ADAF: Advection-dominated accretion flow

AGN: Active galactic nucleus/nuclei

BH: Black hole

BL Lac: BL Lacertae objects

BZ: Blandford-Znajek mechanism

EHT: Event Horizon Telescope

FSRQ: Flat-spectrum radio quasar

GR: General relativity

GRMHD: General relativistic magnetohydrodynamics

GRRMHD: General relativistic radiative magnetohydrodynamics

LAT: Large Area Telescope

MAD: Magnetically arrested disc

MHD: Magnetohydrodynamics

MKS: Modified Kerr-Schild

MPI: Message passing interface

MRI: Magnetorotational instability

NT: Novikov-Thorne

PIC: Particle-in-cell

RIAF: Radiatively innefective accretion flow

SANE: Standard and normal evolution 
SDSS: Sloan Digital Sky Survey

SMBH: Supermassive black hole

SS: Shakura-Sunyaev 


\section{Chapter 1}

\section{The Stage}

If there is a single, unifying theme at the very core of this thesis, it would be black holes. Initially perceived as strange mathematical curiosities with disturbing physical properties, a series of works over most of the 20th century as well as the first quarter of the 21st century have proved their existence beyond any doubt.

Starting with Schwarzschild's solution of Einstein's equations of General Relativity, theoretical work which would later prove essential to black holes continued with the pioneering works of Chandrasekhar (1931) on the limiting mass of white dwarfs, as well as Tolman (1939) and Oppenheimer and Volkoff (1939) on the inevitability gravitational collapse of neutron stars into black holes. Afterwards, a series of theoretical discoveries of many different mathematical and physical properties of black holes occurred through most of the 1960s and 1970s. The realizations that black holes possess spin, ringdown, and are capable of storing and releasing energy, among many other theoretical advancements (Kerr 1963; Thorne 1974; Hawking 1974) - see Misner, Thorne, and Wheeler (1973) for a very extensive source of many of these -, consolidated many of their features and put their mathematical description in firm ground.

On the observational side, the discovery of Cygnus X-1 (Giacconi et al. 1962) through X-ray observations was one of the main hints that black holes were more than mere mathematical objects. Indeed, indirect evidence of the existence of black holes was 


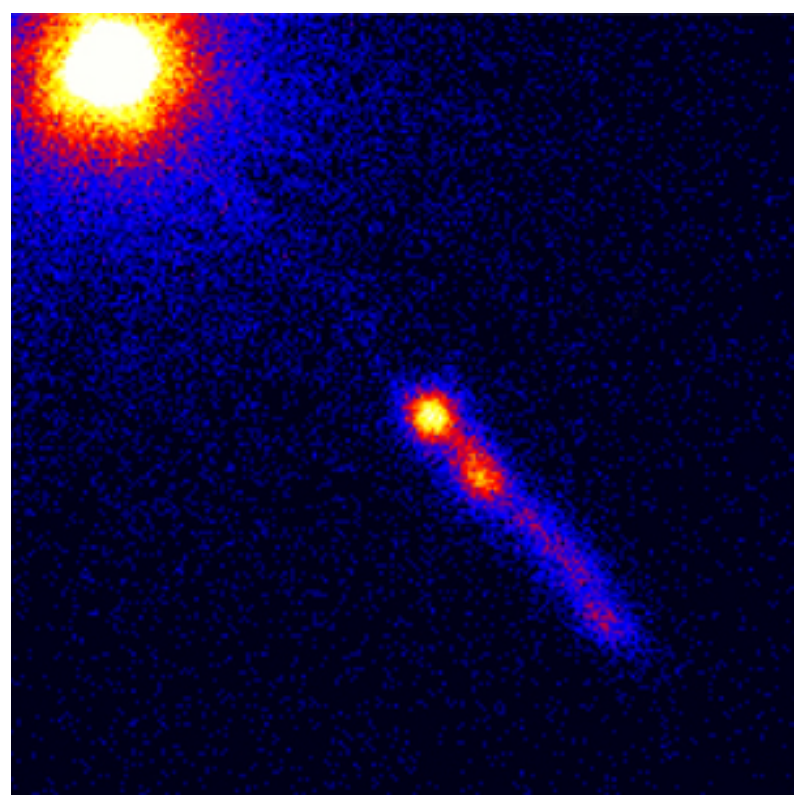

Figure 1.1: The quasar 3C 273 observed in the X-ray by the Chandra X-Ray Observatory, with the jet clearly visible in the center/bottom right. Credit: Herman Marshall/NASA Public Domain - see also Marshall et al. (2001).

starting to appear in the second half of the 20th century, with the discovery of quasar 3C 273 (see fig. 1.1), which was shown by Schmidt (1963) to be at redshift $z=0.16$, and the observations of radio galaxies (Moffet 1966; Bridle and Perley 1984). Also, the motion of stars around a small radio source at the center of the Milky Way (Eisenhauer et al. 2005) could only be explained by the presence of an object of approximately 4 million solar masses, which was either a black hole or a previously undiscovered new astrophysical object. The direct detection of gravitational waves - either from mergers of black holes (Abbott et al. 2016) or neutron stars (Abbott et al. 2017b) - provided even more evidence of their existence, and all doubt was cast aside by the image showing the shadow of the black hole at the center of the galaxy M87 (The EHT Collaboration 2019) (see fig. 1.2).

The series of theoretical and observational studies described very briefly above also culminated in the realization that black holes have a ubiquitous presence in the Milky Way as well as in the universe. For instance, they may be the outcome of the death of a massive star, in which case they will weigh between $\sim 3$ to a few dozens of solar 


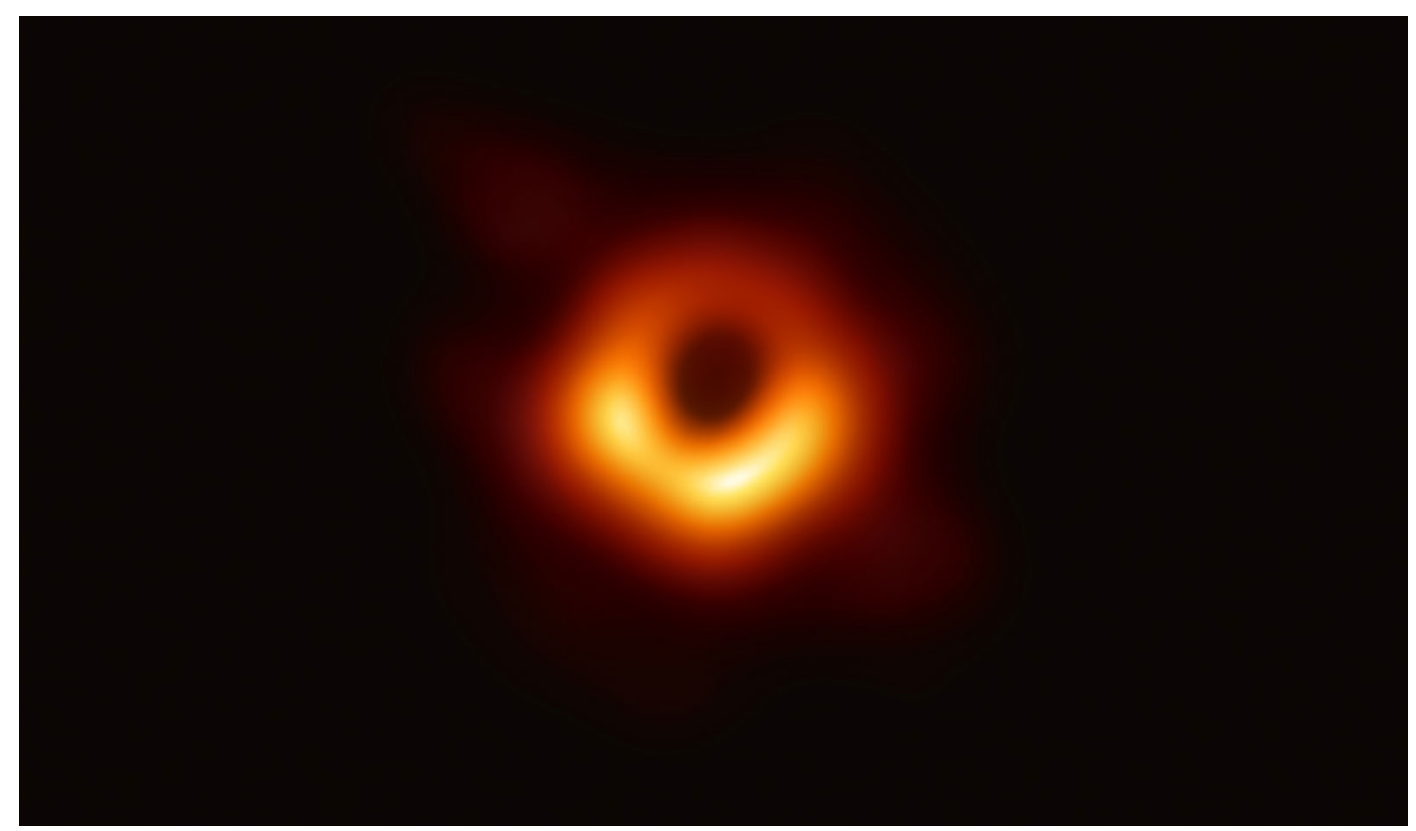

Figure 1.2: Radio image by the Event Horizon Telescope showing the shadow of the black hole at the core of the galaxy M87 and the surrounding accretion disc. Credit: Event Horizon Telescope Collaboration.

masses. However, in order to explain the luminosity of active galactic nuclei (AGN) - over $10^{46} \mathrm{erg} \mathrm{s}^{-1}$ - one would need supermassive black holes (SMBH) weighing between $10^{6}$ to $10^{10}$ solar masses (Rees 1984), as only such a massive object would have an enough amount of gravitational potential energy to be converted into such high luminosities. Along with stellar mass black holes and SMBHs, primordial and intermediate mass black holes ( $10^{2}$ to $10^{5}$ solar masses) are also being subject to extensive research (Miller and Colbert 2004).

Naturally, the study of black holes is a vast field, either in physics, astronomy, astrophysics, mathematics or any combination of these. Among these problems is that of accretion of matter onto black holes.

In the presence of a massive body such as a black hole, the infalling gas that gets captured by its gravitational pull has angular momentum, and is thus prevented from falling directly into the black hole. As a result, this gas forms an accretion disc. As one approaches the black hole, the gravitational pull becomes stronger, thus accelerating the infalling gas to higher velocities, which in turn emits radiation at higher energies, 
from visible light further away from the black hole, to radio and X-rays at the inner parts of the accretion disc. This radiation may be due to thermal processes, but non-thermal processes play a significant role as well, with non-thermal synchrotron emission being required to explain the spectrum of Sagittarius A* (Yuan, Quataert, and Narayan 2003).

Accretion is directly related to another observational feature of black holes. Some active galaxies - defined as galaxies with very high luminosity and activity at their very center -, as well as many other X-ray binaries, also feature a jet, which is detected in radio and sometimes $\mathrm{X}$-ray wavelengths and may extend into many parsecs away from the black hole, in the cases where it is launched by a SMBH at the center of a galaxy. These jets are launched at relativistic speeds, with particles being accelerated and emitting non-thermal synchrotron radiation. Jets, along with winds, have important implications to the evolution of the supermassive black hole's host galaxy, as black hole feedback regulates the amount of available gas necessary to form new stars (Fabian 2012; Kormendy and Ho 2013).

Despite the size, power and influence of the physical processes associated with black holes, they are fundamentally simple objects which can be described by only two parameters: its mass and spin. The latter plays a fundamental role when threaded by magnetic fields brought in by the accretion flow, leading to the emission and launching of jets (Blandford and Znajek 1977), which is not yet fully understood. Fermi acceleration and magnetic reconnection have both been proposed as mechanisms that will accelerate non-thermal electrons, both of which being successful in a few cases (de Gouveia dal Pino and Lazarian 2005; Sironi and Spitkovsky 2014; Khiali, de Gouveia Dal Pino, and del Valle 2015; Sironi, Petropoulou, and Giannios 2015; Khiali and de Gouveia Dal Pino 2016; Sironi, Giannios, and Petropoulou 2016; Petropoulou, Giannios, and Sironi 2016; Petropoulou et al. 2018; Lewis, Finke, and Becker 2018; Lewis, Finke, and Becker 2019; Christie et al. 2019a).

The relation between accretion and jet launching with the two fundamental param- 
eters of black holes can be seen in the association of rapidly rotating black holes with powerful emissions. This relation can be seen in the context of galaxy and SMBH evolution. The growth of SMBHs is expected to occur through essentially two mechanisms: mergers between black holes and accretion of matter onto black holes (Volonteri et al. 2005; Volonteri 2012) - see Gammie, Shapiro, and McKinney (2004) for a study on these physical processes and their effects on black hole spin evolution. Both processes affect significantly the mass and spin of these SMBHs: black holes with large spins before mergers would see a decrease in the spin of the resulting object, while spin would be increased if the black holes had low spins before merging (Volonteri et al. 2013). Accretion, on the other hand, would be responsible for spinning up the black hole as its mass increases, although Thorne (1974) demonstrated that the black hole will accrete the radiation emitted by the disc, thus producing a torque that prevents the spin from going beyond the limiting value of $a=0.998$.

Therefore, the relation between accretion, jet launching and black hole spin is essential to understand observations and supermassive black hole evolution. In view of this, we perform studies which aim at advancing our understanding as to how the jet efficiency is affected by the black hole spin, as well as how the black hole rotation affects the regions in the jet where non-thermal emission might occur. To do so, we use a combination of general relativistic magnetohydrodynamical (GRMHD) simulations and gamma-ray observations from the Fermi Large Area Telescope (LAT), as explained below. 


\section{Thesis overview}

In Chapter 2, we discuss Kerr black holes, active galactic nuclei, accretion discs, and jets, which form the astrophysical backbone of this thesis. In Chapter 3, we will present the equations of GRMHD in the form they are evolved in numerical codes. We will also present the numerical methods behind the GRMHD code used in the simulations.

In Chapter 4, we present a study which combines an existing simulation-based model to relate jet efficiencies and black hole spins with gamma-ray observations by the Fermi-LAT. There, our goal is to test the accuracy of this simulation-model, in order to see if it can produce reliable estimates of black hole spins based on the observed jet efficiency. The objects which we use as a test are flat-spectrum radio quasars (FSRQs), whose accretion discs are characterized by thin accretion discs.

Our simulations are presented in Chapter 5. There, we will discuss the main features of our simulations, as well as the identification and characterization of regions where non-thermal emission might occur. Although our focus is the emission in the jet, we also cover the innermost part of the accretion disc. We wish to compare the effects of different magnetic field topologies and black hole spins to the appearance of current sheets in these systems, so as to have a better understanding of the influence of this fundamental black hole parameter on the sites where non-thermal emission might originate from.

We conclude the thesis in Chapter 6. There, we return to our main results and relate them to existing work in the literature and also present a few possible future developments. 


\section{Chapter 2}

\section{Black Holes in Astrophysics}

\subsection{Introduction}

The first serious treatment of black holes was that of Schwarzschild, who successfully solved the Einstein equations of General Relativity for a spherical, non-rotating mass. Although Schwarzschild's work wasn't itself dedicated to black holes, it laid the foundation for a serious, rigorous treatment of spacetime regions containing gravitational singularities. Since then, further theoretical work and, above all, empirical evidence, changed the status of black holes and they are now understood as real objects capable of influencing not only their immediate surroundings, but also the entire evolution of galaxies, in the case of SMBHs.

In what follows, we will present the main "actors" in this thesis. Starting with Kerr black holes, we will discuss the coordinate metrics used to describe them as well as some physical effects that take place due to black hole rotation. Then, we will give an overview of accretion and jet production, as these two closely associated phenomena are the cornerstone of the active galactic nuclei that we study here. These will be presented afterwards, and will be followed by a brief presentation of the physics of GRMHD and the structure of the code employed in our simulations. 


\subsection{Rotating black holes}

Although theory allows for different types of black holes, astrophysical black holes are understood to be uncharged and rotating, i.e., Kerr black holes. Also, the mass of the accretion disc is negligible compared to the black hole mass, so that any gravitational effects due to the accretion disc are ignored. In what follows, we will discuss some of the main properties of Kerr black holes.

Since Kerr black holes are rotating and possess no electric charge, the only two parameters necessary to describe them are their mass $M$ and their spin $a$. A mathematical description of Kerr black holes is usually done in either Boyer-Lindquist or Kerr-Schild coordinate systems.

These coordinates are useful for the following reasons: The first is that the BoyerLindquist coordinates provides us with a lot of physical insights regarding some of the most fundamental properties of Kerr black holes, which include the event horizon, the ergosphere and frame-dragging. The latter two of these are intimately related to processes of energy extraction from black holes, which are the main proposed explanation for powering relativistic jets. The second reason is that the GRMHD code used in the simulations is written in a coordinate system which is based on the Kerr-Schild coordinates.

\subsubsection{Boyer-Lindquist coordinates}

In spherical Boyer-Lindquist coordinates, the Kerr metric is given by (we set $G=c=1$ )

$$
\begin{aligned}
d s^{2} & =-\left(1-\frac{2 M r}{\Sigma^{2}}\right) \mathrm{dt}^{2}+\frac{\Sigma^{2}}{\Delta} \mathrm{dr}^{2}+\Sigma^{2} \mathrm{~d} \theta^{2} \\
& +\left(r^{2}+a^{2}+\frac{2 M a^{2} r \sin ^{2} \theta}{\Sigma^{2}}\right) \sin ^{2} \theta \mathrm{d} \phi^{2}-\frac{2 M a r \sin ^{2} \theta}{\Sigma^{2}} \mathrm{dtd} \phi
\end{aligned}
$$


where $\Sigma=r^{2}+a^{2} \cos ^{2} \theta$. Upon a quick inspection of Equation (2.2.1), we notice that the Kerr metric possesses a singularity at

$$
\Delta=r^{2}-2 M r+a^{2}=0 .
$$

It can be shown that this is a coordinate singularity, that is, it disappears when we choose another coordinate system - see the Kerr-Schild coordinates below.

Equation (2.2.2) has two solutions,

$$
\begin{aligned}
& r_{-}=M-\sqrt{M^{2}-a^{2}}, \\
& r_{+}=M+\sqrt{M^{2}-a^{2}} .
\end{aligned}
$$

The first of these, which is called inner horizon, is of no interest for us, and will not be considered here ${ }^{1}$, but the second one, which is called outer horizon, is in fact the Kerr black hole event horizon. Hence, from now on, the term "event horizon", will exclusively refer to the outer event horizon.

To see why the outer event horizon is the so called "point of no return", we proceed as follows. Assume a hypersurface ${ }^{2} \mathcal{S}=r_{+}=$const. Then, the normal vector to this hypersurface is given by $n_{\mu}=\partial_{\mu} \mathcal{S}=(0,1,0,0)$. Now, to see if the hypersurface $r_{+}$is timelike, null or spacelike ${ }^{3}$, we simply calculate $n_{\mu} n^{\mu}$ :

$$
n_{\mu} n^{\mu}=n_{\mu} n_{v} g^{\mu v}=g^{r r}=\frac{\Delta}{\Sigma^{2}}
$$

${ }^{1}$ The inner horizon is a Cauchy horizon, which we do not cover here. We also do not cover the $\Sigma=0$ singularity. Both are beyond the limits of the codes which we employ for simulations.

${ }^{2}$ It suffices to give a very informal "definition": a hypersurface can be seen simply as a $(n-1)$ dimensional slice of an $n$-dimensional spacetime.

${ }^{3}$ By definition, a hypersurface $\mathcal{S}$ can be classified according to the normal vectors $n^{\mu}$ to them as follows:

$$
\mathcal{S} \text { is called }\left\{\begin{array}{lll}
\text { spacelike } & \text { if } & n^{\mu} n_{\mu}<0, \\
\text { timelike } & \text { if } & n^{\mu} n_{\mu}>0, \\
\text { null } & \text { if } & n^{\mu} n_{\mu}=0 .
\end{array}\right.
$$


which vanishes for $\Delta=0$. This is precisely our case. Therefore, $r_{+}$is a null surface, that is, it separates regions of spacetime where $r=$ constant are timelike surfaces from regions where they are spacelike surfaces. Now, if $r<r_{+}$, that is, if $\Delta<0$, the hypersurface will be spacelike, whereas for $r>r_{+}(\Delta>0)$ it will be timelike. Hence one can only cross $r_{+}$once, namely from $r>r_{+}$to $r<r_{+}$. This is why $r_{+}$is the event horizon.

As a final remark on the event horizon, we note that setting $a=0$ in Equation (2.2.3) will lead us back to the Schwarzschild radius $r_{\mathrm{Sch}}=2 G M / c^{2}$ (recall we set $G=c=1$ ).

\subsubsection{Kerr-Schild coordinates}

The Kerr-Schild coordinates are closely related to Boyer-Lindquist, except that in this case the coordinate singularity that appears at the outer event horizon in Boyer-Linquist coordinates disappears. In Kerr-Schild coordinates, the Kerr metric is given by

$$
\begin{aligned}
\mathrm{ds}^{2} & =-\left(1-\frac{2 r}{\Sigma}\right) \mathrm{dt}^{2}+\left(\frac{4 r}{\Sigma}\right) \mathrm{drdt}+\left(1+\frac{2 r}{\Sigma}\right) \mathrm{dr}^{2}+\Sigma \mathrm{d} \theta^{2} \\
& +\sin ^{2} \theta\left[\Sigma+a^{2}\left(1+\frac{2 r}{\Sigma}\right) \sin ^{2} \theta\right] \mathrm{d} \phi^{2}-\left(\frac{4 a r \sin ^{2} \theta}{\Sigma}\right) \mathrm{d} \phi \mathrm{dt} \\
& -2 a\left(1+\frac{2 r}{\Sigma}\right) \sin ^{2} \theta \mathrm{d} \phi \mathrm{dr},
\end{aligned}
$$

where, as above, $\Sigma=r^{2}+a^{2} \cos ^{2} \theta$. They serve as a basis for the coordinate system used in the GRMHD code. We will return to this topic in Chapter 3, when we describe the code's main features.

\subsubsection{Ergosphere, frame-dragging, and energy extraction}

Besides the event horizon, which we have discussed above, there are a few other properties of Kerr black holes which are worthy of note. The first of these is the ergosphere, which is a region outside the event horizon in which it is impossible for a 
particle to remain at rest.

We note that the Kerr metric, written in Boyer-Lindquist coordinates, is independent of $t$ and $\phi$, meaning that we may define Killing vectors $\partial_{t}$ and $\partial_{\phi}$ which are associated, respectively, with time-translation invariance and axial symmetry. We may write some of the metric components in terms of these two vectors (Misner, Thorne, and Wheeler 1973), namely $g_{t t}=\partial_{t} \cdot \partial_{t}, g_{t \phi}=\partial_{t} \cdot \partial_{\phi}$, and $g_{\phi \phi}=\partial_{\phi} \cdot \partial_{\phi}$.

A stationary observer is one that does not see a changing spacetime geometry as it moves, which implies that it must move along world lines which are combination of the Killing vectors of the metric, $\partial_{t}$ and $\partial_{\phi}$. In other words, a stationary observer moves along world lines of constant $(r, \theta)$, because if these were to change, it would see a different spacetime geometry around itself. A static observer is an observer whose worldlines $^{4}$ are timelike ${ }^{5}$, i.e., causally connected, with tangent vector parallel to the timelike $\partial_{t}$ Killing vector. Since the world lines of $\partial_{t}$ are constant in $(r, \theta, \phi)$, a static observer moves along world lines of constant $(r, \theta, \phi)$. We note that constant in $\phi$ means zero angular velocity.

For a stationary observer, its angular velocity $\Omega$ and its 4 -velocity $u^{\mu}$ are given, respectively, by

$$
\Omega=\frac{d \phi}{d t}=\frac{u^{\phi}}{u^{t}}
$$

and

$$
u^{\mu}=u^{t} \partial_{t}+u^{\phi} \partial_{\phi}=\frac{\partial_{t}+\Omega \partial_{\phi}}{\sqrt{-g_{t t}-2 \Omega g_{t \phi}-\Omega^{2} g_{\phi \phi}}}
$$

For $u^{\mu}$ above to remain timelike, we need to have $g_{\mu \nu} u^{\mu} u^{v}=-1$, which implies (Misner,

${ }^{4}$ The worldline of an object is its path in a spacetime characterized by a metric $g_{\mu v}$.

${ }^{5}$ In a spacetime defined by $g_{\mu v}$, a vector $u^{\mu}$ which is tangent to a worldline is classified as follows:

$$
u^{\mu} \text { is called } \begin{cases}\text { spacelike } & \text { if } g_{\mu \nu} u^{\mu} u_{v}>0, \\ \text { timelike } & \text { if } g_{\mu \nu} u^{\mu} u_{v}<0, \\ \text { null } & \text { if } g_{\mu \nu} u^{\mu} u_{v}=0 .\end{cases}
$$


Thorne, and Wheeler 1973) in

$$
g_{t t}+2 \Omega g_{t \phi}+\Omega^{2} g_{\phi \phi}<0
$$

We find that the angular velocity of stationary observers is constrained such that

$$
\Omega_{\min } \equiv \omega-\sqrt{\omega^{2}-\frac{g_{t t}}{g_{\phi \phi}}}<\Omega<\Omega_{\max } \equiv \omega+\sqrt{\omega^{2}-\frac{g_{t t}}{g_{\phi \phi}}},
$$

where

$$
\omega=-\frac{g_{t \phi}}{g_{\phi \phi}}
$$

Now, examining $\omega$, we have

$$
\omega=-\frac{g_{t \phi}}{g_{\phi \phi}}=\frac{2 M r a}{\left(r^{2}+a^{2}\right)^{2}-\Delta a^{2} \sin ^{2} \theta}
$$

which means that $\Omega_{\min }$ must increase as $r$ decreases. In other words, we may say that the frame is being dragged by the gravitational pull and rotation of the black hole.

On the other hand, if $g_{t t}=0$, then $\Omega_{\min }=0$. This implies that all stationary observers must orbit the black hole with positive angular velocity. An immediate consequence of this frame dragging effect applies for static observers: since there is no way for an observer not to move, there can no longer be static observers once this radius is reached. The radius for which $g_{t t}=0$, which is called the static limit, is given by

$$
r=r_{0}(\theta)=M+\sqrt{M^{2}-a^{2} \cos ^{2} \theta}
$$

At and inside the surface limited by Equation (2.2.12), no static observers may exist. The static limit is the outer border of the ergosphere, with the inner border being the event horizon, Equation (2.2.3). Note that, at the poles $(\cos \theta=0)$, the static limit coincides with the event horizon, i.e. the ergosphere inner and outer limits both coincide at the 
poles.

Inside the ergosphere, it is possible for particles to have negative energy, as measured by an observer at infinity. The Penrose process (Penrose 1969) allows for the extraction of energy of a rotating black hole. A particle with energy $E>0$ (as measured by an observer at infinity) might decay into two particles. The first of these particles has positive energy, and is thus able to escape to infinity, while the second falls into the black hole. Penrose showed that, in this scenario, this outgoing particle has a larger net energy than the original particle. Since more energy was extracted than produced, there occurred a transfer of energy from the black hole to the particle. Hence, energy has been extracted from the black hole.

In astrophysics, the Blandford-Znajek (BZ) mechanism (Blandford and Znajek 1977) can be seen as a realization of the Penrose process, in which the magnetic fields play a determining role in guiding the particles away from the black hole and thus extracting its rotational energy. We shall discuss the BZ mechanism when we discuss the formation and propagation of jets (Section 2.3.4), but before doing so we must present the astrophysical context in which they appear.

\subsection{Accretion discs and jets}

As matter approaches a black hole, angular momentum forces the gas to settle into a circular, disc-like structure - an accretion disc. Modeling accretion discs has been of great interest for astrophysicists willing to explain the observational features of different types of active galactic nuclei (AGN) - AGN are presented in Section 2.4. The most well-known model is the thin Shakura and Sunyaev (1973) disc (also known as the " $\alpha$-prescription"), which assumes that turbulence in the accretion flow is responsible 
for an increased viscosity $v$ given by

$$
v=\alpha c_{s} H,
$$

where $c_{\mathrm{s}}$ is the sound speed and $H$ is the scale height of the disc, and $\alpha$ is a free parameter such that $0<\alpha \lesssim 1$. The parameter $\alpha$ is present because, in the original work of Shakura and Sunyaev (1973), the mechanism responsible for viscosity was not fully understood, so our ignorance regarding the viscosity mechanism was transferred from $v$ to $\alpha$. A general relativistic version of the Shakura-Sunyaev disc is the Novikov and Thorne (1973) model.

The central role played by turbulence in accretion is manifested in the magnetorotational instability (MRI, (Balbus and Hawley 1991; Balbus and Hawley 1998)), without which accretion cannot occur. In MRI, two orbiting masses are connected by a magnetic field, but if one of the masses is at a lower orbit than the other, the fields stretch as angular velocity decreases with radius, which forces the mass at lower orbit to pull ahead of the other mass. As a consequence, the magnetic tension between these two masses increases and forces them to return to their original separations. However, in doing so, the inner mass transfers angular momentum to the outer mass, dropping to a lower orbit and increasing the magnetic tension. This process leads to unstable and turbulent motion, and forces accretion to occur as masses drop to lower orbits.

Accretion discs are classified according to a few parameters, such as disc thickness $h$, accretion rate $\dot{M}$, optical depth $\tau$ and accretion efficiency $\varepsilon$. In accordance with these properties, discs may be classified ${ }^{6}$ as thick, thin, Polish doughnut, slim, or advectiondominated accretion flows (ADAFs), also called radiatively inefficient accretion flows (RIAFs). Of these parameters, the accretion rate $\dot{M}$ is the most fundamental. It is often

${ }^{6}$ This classification is by no means complete. We focus only on the disc models that are of interest for this thesis. A good review of black hole accretion, which includes accretion disc models, can be found in Abramowicz and Fragile (2013). 
expressed in terms of the Eddington rate

$$
\dot{M}_{\mathrm{Edd}} \equiv \frac{L_{\mathrm{Edd}}}{\varepsilon c^{2}}
$$

where $L_{\mathrm{Edd}}$ is the Eddington luminosity, which is the luminosity of a body where gravity and radiation pressure are in equilibrium, and is given by

$$
L_{\mathrm{Edd}} \equiv \frac{4 \pi G M m_{\mathrm{p}} c}{\sigma_{\mathrm{T}}}=1.26 \times\left(\frac{M}{M_{\odot}}\right) \mathrm{erg} \mathrm{s}^{-1}
$$

In the above equations, $\varepsilon$ is generally assumed to be around $0.1, m_{\mathrm{p}}$ is the proton mass and $\sigma_{\mathrm{T}}$ is the Thomson scattering cross-section.

Although the accretion discs mentioned above are all reasonable choices when modeling different phenomena in nature, we shall direct our discussion towards only two of them, both of which are sub-Eddington. These are the thin discs and hot ADAFs, which are described in broad terms in the following two sections. This choice is simply because these are the accretion discs that will be featured in the following chapters. For instance, our study of spins and jet efficiencies of flat-spectrum radio quasars (FSRQs), which is presented in Chapter 4, takes into account only thin discs, as they are believed to be the preferred state of accretion discs in FSRQs. Our GRMHD simulations, presented in Chapter 5, evolve ADAFs.

\subsubsection{Thin discs}

As matter is accreted towards a black hole, energy is dissipated from the disc as radiation. If the gas is able to immediately radiate this energy away, the disc will cool (radiative cooling), and its vertical size diminishes greatly, thus forming a geometrically thin accretion disc, with the height to radius ratio being $H / R \lesssim 0.3$. In thin discs, the gas temperature is around $10^{4}-10^{7} \mathrm{~K}$, as much of the energy is dissipated away. These 
discs are radiatively efficient, optically thick, and radiate a blackbody-like thermal radiation. Their luminosity is $\gtrsim 10^{-3} L_{\text {Edd }}$. Since luminosity is related to accretion rate $\dot{M}$ by Equation (2.3.2), one may also describe a thin disc by its Eddington accretion rate, $\dot{M}_{\text {Edd }}$. Although it is customary to assume an efficiency parameter $\varepsilon \sim 0.1$, thin discs can be very efficient systems, as $\varepsilon$ becomes larger than 0.1 .

The Novikov and Thorne (1973) (NT) model for thin disc radiation efficiency is perhaps the most well-known description of a thin disc in the framework of general relativity. It has been successfully employed to describe a series of systems - both in stellar mass black holes and in AGN -, and it has been used to estimate the spins of stellar black holes (Shafee et al. 2006; McClintock et al. 2006), although simulations have suggested (Avara, McKinney, and Reynolds 2016) that strong magnetic fields near the black hole may lead to deviations from the NT model by achieving a radiative efficiency $80 \%$ higher than predicted by the NT model for a moderately spinning black hole.

\subsubsection{A thin disc solution}

Here, we describe the main features of a thin accretion disc around a black hole a full treatment can be found in Frank, King, and Raine (2002). Since its mass is low compared to the black hole, we neglect disc self-gravity effects and assume the gravitational potential is entirely dominated by the black hole. The vertical structure of a thin disc with total pressure $P$, around a black hole with mass $M$, at a distance $R$ from the black hole and height $z$ from the disc plane, can be obtained assuming hydrostatic equilibrium,

$$
\frac{1}{\rho} \frac{\partial P}{\partial z}=\frac{\partial}{\partial z}\left[\frac{G M}{\left(R^{2}+z^{2}\right)^{1 / 2}}\right]
$$

which becomes, upon setting $z \ll R$ (valid for a thin disc)

$$
\frac{1}{\rho} \frac{\partial P}{\partial z}=-\frac{G M z}{R^{3}} .
$$


The total pressure is related to the gas density $\rho$ by $P \sim \rho c_{s}^{2}$, where $c_{s}$ is the speed of sound, while the disc height is denoted by $H$. Approximating $\partial P / \partial z$ by $P / H$ and $z$ by $H$, we find

$$
\frac{H}{R} \cong c_{S}\left(\frac{R}{G M}\right)^{1 / 2} \ll 1,
$$

which implies in

$$
c_{S} \ll\left(\frac{G M}{R}\right)^{1 / 2}
$$

By solving the radial component of the Euler equation

$$
v_{R} \frac{\partial v_{R}}{\partial R}-\frac{v_{\phi}^{2}}{R}+\frac{1}{\rho} \frac{\partial P}{\partial R}+\frac{G M}{R^{2}}=0
$$

it can be shown (Frank, King, and Raine 2002) that, if Equation (2.3.7) holds, the angular velocity $v_{\phi}$ will be

$$
v_{\phi}=\left(\frac{G M}{R}\right)^{1 / 2}\left[1+O\left(\mathcal{M}^{-2}\right)\right]
$$

where $\mathcal{M} \equiv v_{\phi} / c_{s}$ is the Mach number. Comparing Equations (2.3.7) and (2.3.9), we conclude that, in a thin disc, $v_{\phi}$ is very supersonic. Now, if we assume that the total pressure is a sum of gas and radiation pressures, both of which depend on the gas temperature $T_{c}$, we conclude that $v_{\phi} \gg c_{S}$ is ultimately intrinsically related to the cooling mechanism which acts on the disc.

The Shakura and Sunyaev (1973) disc - see Equation (2.3.1) - is a simple thin disc solution in which the disc surface area $\Sigma$, disc height $H$, gas density $\rho$, temperature $T_{c}$, optical thickness $\tau$, viscosity $v$ and radial velocity $v_{R}$ are related to the accretion rate $\dot{M}$, the radius $R$, black hole mass $M$ and the $\alpha$ parameter by

$$
\begin{aligned}
& \Sigma=5.2 \alpha^{-4 / 5} \dot{M}_{16}^{7 / 10} m_{1}^{1 / 4} R_{10}^{-3 / 4} f^{14 / 5} \mathrm{~g} \mathrm{~cm}^{-2} \\
& H=1.7 \times 10^{8} \alpha^{-1 / 10} \dot{M}_{16}^{3 / 20} m_{1}^{-3 / 8} R_{10}^{9 / 8} f^{3 / 5} \mathrm{~cm} \\
& \rho=3.1 \times 10^{-8} \alpha^{-4 / 10} \dot{M}_{16}^{11 / 20} m_{1}^{5 / 8} R_{10}^{-15 / 8} f^{11 / 5} \mathrm{~g} \mathrm{~cm}^{-3}
\end{aligned}
$$




$$
\begin{aligned}
T_{c} & =1.4 \times 10^{4} \alpha^{-1 / 5} \dot{M}_{16}^{3 / 10} m_{1}^{1 / 4} R_{10}^{-3 / 4} f^{6 / 5} \mathrm{~K} \\
\tau & =190 \alpha^{-4 / 5} \dot{M}_{16}^{1 / 5} f^{4 / 5} \\
v & =1.8 \times 10^{14} \alpha^{4 / 5} \dot{M}_{16}^{3 / 10} m_{1}^{-1 / 4} r_{10}^{3 / 4} f^{6 / 5} \mathrm{~cm}^{2} \mathrm{~s}^{-1} \\
v_{R} & =2.7 \times 10^{4} \alpha^{4 / 5} \dot{M}_{16}^{3 / 10} m_{1}^{-1 / 4} R_{10}^{-1 / 4} f^{-14 / 5} \mathrm{~cm} \mathrm{~s}^{-1},
\end{aligned}
$$

where $f=\left[1-\left(R / R_{*}\right)^{0.5}\right]^{0.25}\left(R_{*}\right.$ is the surface radius of the central object), $\dot{M}_{16}=$ $\dot{M} /\left(10^{16} \mathrm{~g} \mathrm{~s}^{-1}\right), m_{1}=M / M_{\odot}$, and $R_{10}=R /\left(10^{10} \mathrm{~cm}\right)$. This is an useful prescription, because it reduces a set of complicated differential equations - see, for example, Abramowicz and Fragile (2013) - into a simpler system of algebraic equations.

\subsubsection{Advection-dominated accretion flows (ADAFs)}

Unlike thin discs, ADAFs are dominated by another mechanism for cooling: advection. Instead of being radiated away, the dissipated energy is advected into the black hole. ADAFs can be subdivided into cold and hot. The former is characterized by $\dot{M}$ values approaching $\dot{M}_{\mathrm{Edd}}$, and luminosities $L \leq 0.3 L_{\mathrm{Edd}}$. At such higher accretion rates, the gas becomes optically thick $(\tau \gg 1)$ and is unable to radiate the dissipated energy, which results in radiation being trapped (long diffusion time: $t_{\text {diff }} \gg t_{\text {acc, }}$ where $t_{\text {acc }}$ is the accretion time) and advected into the black hole (Narayan, Mahadevan, and Quataert 1998). As a result, radiative efficiency becomes smaller than $0.1 \dot{M} c^{2}$. These ADAFs are called slim discs. It should be noted, however, that the gas is cooled, resulting in temperatures comparable to those found in thin discs - hence, these are called cold ADAFs.

In contrast to cold ADAFs, hot ADAFs have lower accretion rates, higher temperatures, and are optically thin - see Yuan and Narayan (2014) for a comprehensive review of hot accretion flows. In hot ADAFs, the retention of energy in the flow implies in higher pressure, which in turn implies in higher speed of sound $c_{s}$ and also in a geomet- 
rically thicker disc (Narayan and Yi 1995; Narayan, Mahadevan, and Quataert 1998). Besides, standard accretion theory predicts that the radial velocity $v_{r}$ is proportional to both the disc thickness $H / R$ and $c_{s}$ (Abramowicz et al. 1988; Narayan and Yi 1994). The higher radial velocity implies in a shorter accretion time, $t_{\mathrm{acc}}$, and the gas is unable to radiate its energy in less than $t_{\mathrm{acc}}$. That is, the cooling time is longer than the accretion time, $t_{\text {cool }} \gg t_{\text {acc }}$.

With respect to disc temperatures, these are higher in hot ADAFs because the dissipated accretion energy heats the accretion flow instead of being radiated away. They are usually described by two-temperature models (Narayan and Yi 1995), and the ion temperature approaches $10^{12} \mathrm{~K}$ at the core, while the electron temperature remains a few orders of magnitude lower, at 10 ${ }^{9-11}$ K (Narayan and Yi 1995; Narayan, Mahadevan, and Quataert 1998). Due to the high temperatures in a hot ADAF, pressure leads to an expansion of the gas, which decreases its density and thus reduces its optical depth. Thus, hot ADAFs are optically thin $(\tau \ll 1)$, and are also called radiatively ineffective accretion flows (RIAFs). Here, we will use the terms ADAF and RIAF interchangeably, meaning that, from now on, whenever we mention ADAFs, it will be assumed that the accretion flow is hot. The radiative inefficency of ADAFs allows them to be described by models for which radiation is dynamically unimportant, such as those simulated in many GRMHD codes (Gammie, McKinney, and Tóth 2003).

ADAFs are often employed to describe systems where the luminosity is low, such as Sgr $\mathrm{A}^{*}$, which is a low-luminosity AGN with $L \sim 10^{35-36} \mathrm{erg} \mathrm{s}^{-1}$ (Narayan, Yi, and Mahadevan 1995; Yuan, Quataert, and Narayan 2003). The low luminosity of ADAFs contrasts with that of many systems where accretion is described by thin discs, such as quasars. This is due to the low efficiency of ADAFs, which accrete at lower rates than thin discs, $\dot{M} \lesssim 10^{-3} \dot{M}_{\mathrm{Edd}}$ - for comparison, Faraday rotation measures of Sgr A* give an upper limit to its accretion rate at $\sim 10^{-7} \dot{M}_{\mathrm{Edd}}$ (Marrone et al. 2007).

The spectrum of an ADAF is dominated by synchrotron emission, bremsstrahlung 
and Comptonization (Yuan and Narayan 2014). We show in Figure 2.1 the spectral energy distribution (SED) of Sgr A* modelled by a hot ADAF.

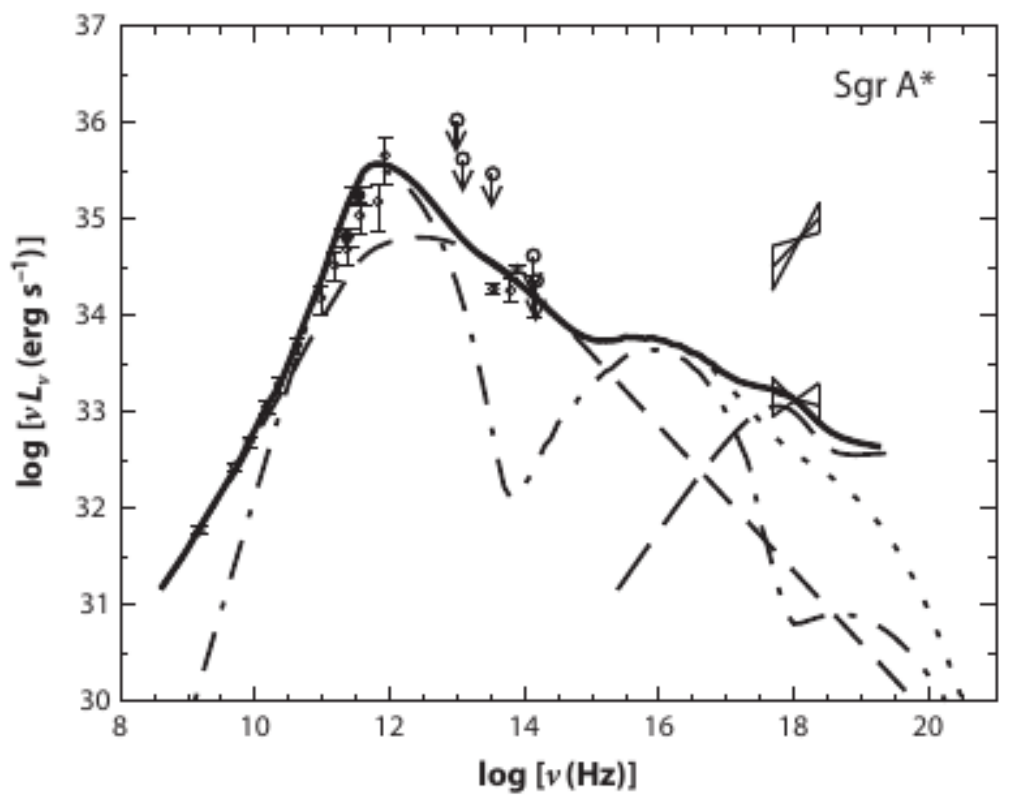

Figure 2.1: Spectral energy distribution of Sgr A* according to a hot ADAF model. Circles with error bars are radio and milimiter wavelengths, circles with arrows are IR upper limits and bowties correspond to X-ray data. The dot-dashed line corresponds to synchrotron emission and the Compton humps from thermal electrons, the shortdashed line shows synchrotron emission from nonthermal electrons, and the longdashed line indicates bremsstrahlung from electrons close to the Bondi radius. The ADAF model spectrum is the thick solid line, which is a sum of the three components mentioned above, and the dotted line shows the total synchrotron and inverse Compton emission. Source: (Yuan and Narayan 2014).

\subsubsection{Jets}

The development of radio astronomy allowed the discovery of many radio sources. Some of these were identified as jets extending to sub-AU scales up to hundreds of thousands of parsecs from their source (Blandford, Meier, and Readhead 2019). Although we focus on jets originating from black holes at the center of an AGN, such as in M87, jets also appear in microquasars (Gallo 2010), protostars (Bally 2016), neutron stars and X-ray binaries (Migliari and Fender 2006; Fender, Homan, and Belloni 2009).

Jets are believed to be intimately related to accretion discs, as the accreting gas 
loses angular momentum and is thus ejected away from the disc plane, either as highly collimated and magnetized relativistic jets, or as winds.

In $\mathrm{AGN}$, jets are common in radio galaxies - such as Centaurus $\mathrm{A}$, where it can also be seen in X-rays (Hardcastle et al. 2003) -, quasars - such as 3C 273 (Marshall et al. 2001) -, and blazars, which are defined as AGN whose jets are pointing towards the observer (Urry and Padovani 1995). Through AGN feedback, jets are able to shut off star formation in their host galaxy by heating up or removing its cold gas, thus being a determining contributor to galaxy evolution (Fabian 2012; Kormendy and Ho 2013).

Some jets in quasars or blazars display apparent speeds larger than the speed of light. This apparent superluminal motion is due to the jets' very high speeds (albeit smaller than $c$ ), characterized by Lorentz factors in the range $2 \lesssim \Gamma \lesssim 30$, as measured in VLBI observations (Kellermann et al. 2004), and small angles to the observer (Rees 1966). Another effect that can be seen in jets is a change in their brightness due to relativistic beaming. Their brightness might also change - in either short or long timescales - according to the viewing angle. This variability is one of the main features of blazars.

\section{The Blandford-Znajek mechanism}

The Blandford-Znajek mechanism (BZ, Blandford and Znajek 1977) is similar to the Penrose process (Penrose 1969) in that it provides a mechanism to extract energy from black holes. For the BZ mechanism to occur, an essential component is a strong magnetic field. Although black holes themselves cannot have their own intrinsic magnetic fields, the accretion disc around them is able to provide enough magnetic flux to power jets.

In $\mathrm{BZ}$, the frame-dragging effect in the ergosphere causes magnetic field lines to tangle around the black hole. We denote the magnetic flux threading the black hole horizon by $\Phi_{\mathrm{BH}}$. As plasma falls into the black hole (analogously to the ingoing particle in the Penrose process), it forces the rotational energy of the black hole to decrease. 
However, as the magnetic field lines tangle around the black hole, they expand due to magnetic pressure, dragging along any plasma coupled to them and thus transferring the rotational energy of the black hole to the outgoing plasma, which propagates away from the black hole in the form of two collimated relativistic jets. The rate at which energy is extracted from a black hole with mass $M$ and rotating with spin $a$ is expressed as (Blandford and Znajek 1977; Tchekhovskoy, Narayan, and McKinney 2010)

$$
P_{\mathrm{BZ}}=\frac{\kappa}{4 \pi c} \Omega_{\mathrm{H}}^{2} \Phi_{\mathrm{BH}}^{2} f\left(\Omega_{\mathrm{H}}\right),
$$

where $\kappa$ is a numerical constant around $0.04-0.05, \Omega_{\mathrm{H}}=a_{*} c / 2 r_{H}$ is the angular frequency of the black hole horizon, $r_{H}=r_{g}\left(1+\sqrt{1+a_{*}^{2}}\right)$ is the horizon radius, $r_{g}=$ $G M / c^{2}$ is the gravitational radius of the black hole. In the original work of $\mathrm{BZ}$, the function $f\left(\Omega_{H}\right)$ was set to unity, but Tchekhovskoy, Narayan, and McKinney (2010) have shown in a more recent perturbative study that this value holds up to spins $a \approx 0.95$, and can be approximated by $f\left(\Omega_{H}\right) \approx 1+1.38\left(\Omega_{H} r_{g} / c\right)^{2}-9.2\left(\Omega_{H} r_{g} / c\right)^{4}$ at even larger spins. Thus, Equation (2.3.10) demonstrates the direct dependence of the extracted power via Poynting flux and the black hole rotation.

\subsection{Active Galactic Nuclei}

At the very center of some galaxies - known as active galaxies - there is a compact region with a radius of the order of 1 parsec possessing such a high luminosity - up to $\sim 10^{48} \mathrm{erg} \mathrm{s}^{-1}$ (Fan et al. 2019) -, that it cannot be explained by star emission. Although there is no universal consensus regarding a precise definition of active galactic nuclei (AGN), here we follow Netzer (2015) and define an AGN as the central region of a galaxy containing a SMBH with at least $10^{5} M_{\odot}$ and a luminosity $L_{\mathrm{AGN}} \geq 10^{-5} L_{\mathrm{Edd}}$, where the Eddington luminosity $L_{\text {Edd }}$ has been defined in Equation (2.3.3). For comparison, the luminosity of AGN can be more than $10^{6}$ times higher than that of an inactive galaxy 
such as the Milky Way, which is no brighter than $10^{-7} L_{\text {Edd }}$ (Marrone et al. 2007).

The high luminosity of AGN is associated with the presence of a rotating accretion disc composed of plasma which is gravitationally bound to the central black hole. In some AGN, jets of particles originating at the AGN core have also been observed in different wavelengths, from radio to $\gamma$-rays (Madejski and Sikora 2016; Walker et al. 2018; Blandford, Meier, and Readhead 2019). Besides covering the entire electromagnetic spectrum, nuclear activity in galaxies is a candidate for the source of very high energy cosmic rays (Pierre Auger Collaboration et al. 2007; Pierre Auger Collaboration et al. 2008; George et al. 2008). We discuss accretion discs and jets in some detail in Section 2.3.

AGN are divided into many different categories, and different authors provide slightly different classifications and/or categories, effectively forming an "AGN zoo" (Tadhunter 2008; Padovani et al. 2017). Historically, the primary observational properties used to classify AGN are their nuclear luminosity, radio loudness and the presence or absence of broad emission lines in their spectra (e.g. Seyfert 1 vs Seyfert 2). Other relevant properties include the radio morphology (e.g. FR I vs FR II), variability and spectral shape (e.g. BL Lacs vs FSRQs - see our discussion of blazars in Section 2.4.3). We show in Table 2.1 a classification of AGN according to optical properties.

Another way of classifying AGN is through their radio loudness. Following Sikora, Stawarz, and Lasota (2007), the radio loudness can be defined as $\mathcal{R} \equiv L_{v_{5}} / L v_{B}=$ $1.36 \times 10^{5}\left(L_{R} / L_{B}\right)$, where $L_{R} \equiv v_{5} L_{v_{5}}, L_{v_{5}}$ is the radio luminosity at $v_{5}=5 \mathrm{GHz}$ and $L_{v_{B}}$ is the luminosity at $\lambda_{B}=4400$ in the B-band.

From the point of view of radio loudness, AGN are often divided into two broad categories, which differ from each other according to the emission from the jets and radio lobes that form on both sides of the galaxy. Radio-loud AGN are characterized by significant contributions from jet and lobe emission to the object's overall luminosity. They are often elliptical galaxies, and some examples include radio galaxies and blazars. 
Table 2.1: Classification of AGN according to their optical properties. Adapted from Blandford, Meier, and Readhead (2019).

\begin{tabular}{|c|c|c|}
\hline Name & Properties & $\begin{array}{c}L_{\text {bol }} \\
\left(\text { erg s}^{-1}\right)\end{array}$ \\
\hline $\begin{array}{l}\text { Low-ionization nuclear emission- } \\
\text { line region (LINER) }\end{array}$ & $\begin{array}{l}\text { Weak emission lines except for low- } \\
\text { ionization lines }\end{array}$ & $<10^{42}$ \\
\hline Seyfert type 1 & $\begin{array}{l}\text { Broad permitted and narrow for- } \\
\text { bidden lines }\end{array}$ & $>10^{42}$ \\
\hline Seyfert type 2 & $\begin{array}{l}\text { Narrow permitted and forbidden } \\
\text { lines }\end{array}$ & $>10^{42}$ \\
\hline Quasi-stellar object (QSO) & Starlike, but very high luminosity & $>10^{45}$ \\
\hline Weak-line radio galaxy (WLRG) & $\begin{array}{l}\text { Analogous to LINER, but radio } \\
\text { emission }\end{array}$ & $<10^{42}$ \\
\hline Narrow-line radio galaxy (NLRG) & Radio galaxy, similar to Seyfert 1 & $>10^{42}$ \\
\hline Broad-line radio galaxy (BLRG) & Radio galaxy analogous to Seyfert 2 & $>10^{42}$ \\
\hline Quasi-stellar radio source (QSR) & QSO with strong radio emission & $>10^{45}$ \\
\hline
\end{tabular}

Table 2.2: Radio-loud AGN classified according to their radio properties and bolometric luminosities, as seen in Blandford, Meier, and Readhead (2019).

\begin{tabular}{l|l|c}
\hline Name & Properties & $\begin{array}{c}L_{\mathrm{bol}} \\
\left(\mathrm{erg} \mathrm{s}^{-1}\right)\end{array}$ \\
\hline $\begin{array}{l}\text { Fanaroff-Riley type I (FRI) } \\
\text { Fanaroff-Riley type II (FRII) }\end{array}$ & $\begin{array}{l}\text { Brighter towards center } \\
\text { Brighter towards lobes } \\
\text { Compact radio source, polarized } \\
\text { optical continuum, weak or absent } \\
\text { emission lines }\end{array}$ & $<10^{42}$ \\
Flat-spectrum radio quasar (FSRQ) & $\begin{array}{l}\text { Compact radio source, strong } \\
\text { quasar-like emission lines }\end{array}$ & $>10^{42}$ \\
\hline
\end{tabular}

Radio-quiet AGN, on the other hand, display insignificant emission from the jets and lobes. They are generally spiral galaxies, and include LINERs and Seyfert galaxies. Finally, quasars might be either radio-loud or radio-quiet.

For the purposes of this thesis, we focus our attention on radio-loud AGN, which are summarized in Table 2.2 - see also Tadhunter (2016) for an extensive review of radio AGN. In particular, we shall direct a significant part of our study to blazars. 


\subsubsection{Radio galaxies}

Radio galaxies show strong polarized and smooth emission at radio wavelengths, which is due to synchrotron processes (Tadhunter 2016). Structurally, they feature radio lobes which may be much larger than their host galaxy, and are almost always elliptical galaxies (Tadhunter 2016). They are further subdivided into two categories, namely FanaroffRiley I (FR I) and Fanaroff-Riley II (FR II) (Fanaroff and Riley 1974). This distinction is based on their radio emission at larger scales. FR I galaxies are brighter towards their central parts, while FR II are brighter towards the lobes, and more luminous than FR I galaxies. This difference is illustrated in Figure 2.2 - see also Meisenheimer et al. (1989).

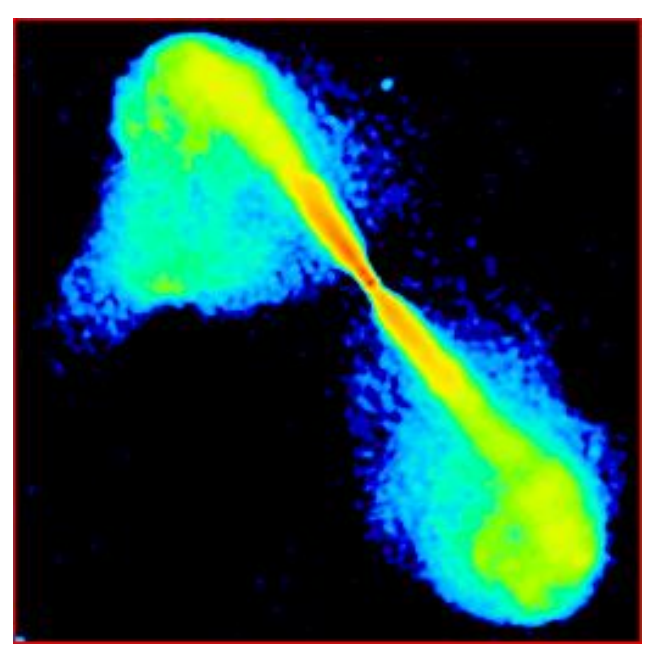

(a) 3C 296, an FR I radio galaxy.

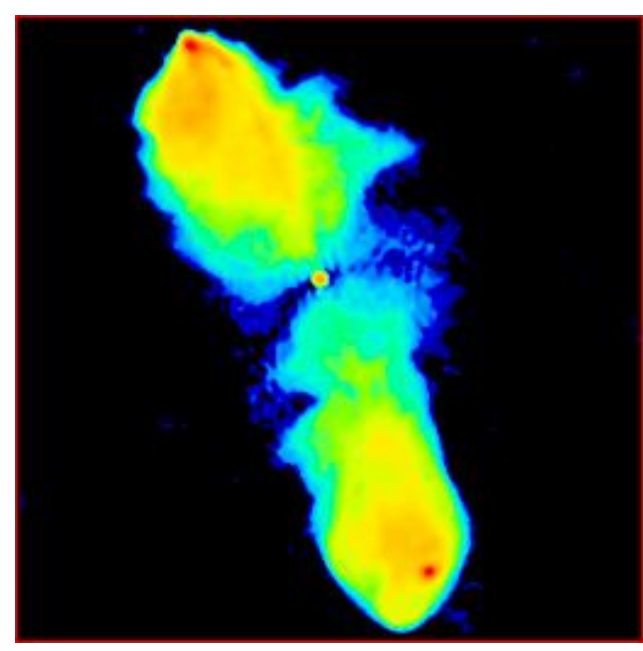

(b) 3C 98, an FR II radio galaxy.

Figure 2.2: Radio images of a Fanaroff-Riley type I (left) and Fanaroff-Riley type II (right) galaxy. The image of 3C 296 shows very clearly the higher brightness towards the central part of the galaxy, where it appears redder. The peak brightness in 3C 98 is seen at the end of the lobes. The colored images were taken from http://www. $j b$. man.ac.uk/atlas/. The image on the left appears on Leahy and Perley (1991), while the image on the right is unpublished and credited to A. H. Bridle.

\subsubsection{Radio-loud quasars}

Quasars have very high luminosities $-L_{\mathrm{bol}}>10^{45} \mathrm{erg} \mathrm{s}^{-1}$, see Table $2.1-$, and the most luminous objects falling into this category - sometimes called quasi-stellar objects (QSOs) if they are radio-quiet - inhabit very massive elliptical galaxies, but they may also be 
spirals. Quasars are more luminous that Seyfert 1s, and their spectra reveal optical and X-ray emission, as well as broad and narrow optical emission lines. If a jet is present, they are called radio-loud quasars. Quasars are brighter and appear at higher redshifts - they have been observed in redshifts up to $\sim 7$ (Venemans et al. 2017). This makes them older than Seyferts.

\subsubsection{Blazars}

Blazars are extremely luminous sources, and their most distinctive feature is that they have relativistic jets which are pointing in the direction of the observer (Urry and Padovani 1995). As a result, the observed brightness and fluxes undergo relativistic beaming and Doppler shift. A consequence of beaming is that the jet radiation may overwhelm the rest of blazar emission, therefore dominating the observed spectrum. Also, another consequence of relativistic effects is that blazar emission may appear superluminal - as an example, the jet component of $1633+382$ was measured to be moving outward at $\sim 6 c$ (Barthel et al. 1995).

Blazars are characterized by highly variable emission, with timescales varying between minutes and weeks (Böttcher et al. 2013). This variability has been observed across the entire electromagnetic spectrum: radio (Hughes 1965), optical (Schmidt 1963), X-rays (Schreier, Gorenstein, and Feigelson 1982) and $\gamma$-rays (Bignami et al. 1981). In particular, the highly variable radio emission in blazars is associated with a flat radio spectrum, with $\alpha \leq 0.5, f_{v} \propto v^{-\alpha}$, where $v$ is the frequency and $\alpha$ is the spectral index. Another distinctive feature of blazars is their spectral energy distribution (SED). These display two peaks: the first is often located between radio and X-ray wavelengths, and is due to synchrotron radiation. The second, high-frequency peak, is centered at gamma-ray wavelengths and results from inverse Compton scattering. Figure 2.3 shows one such example.

The two peaks of a blazar SED appear to be connected by a relation deemed "blazar 


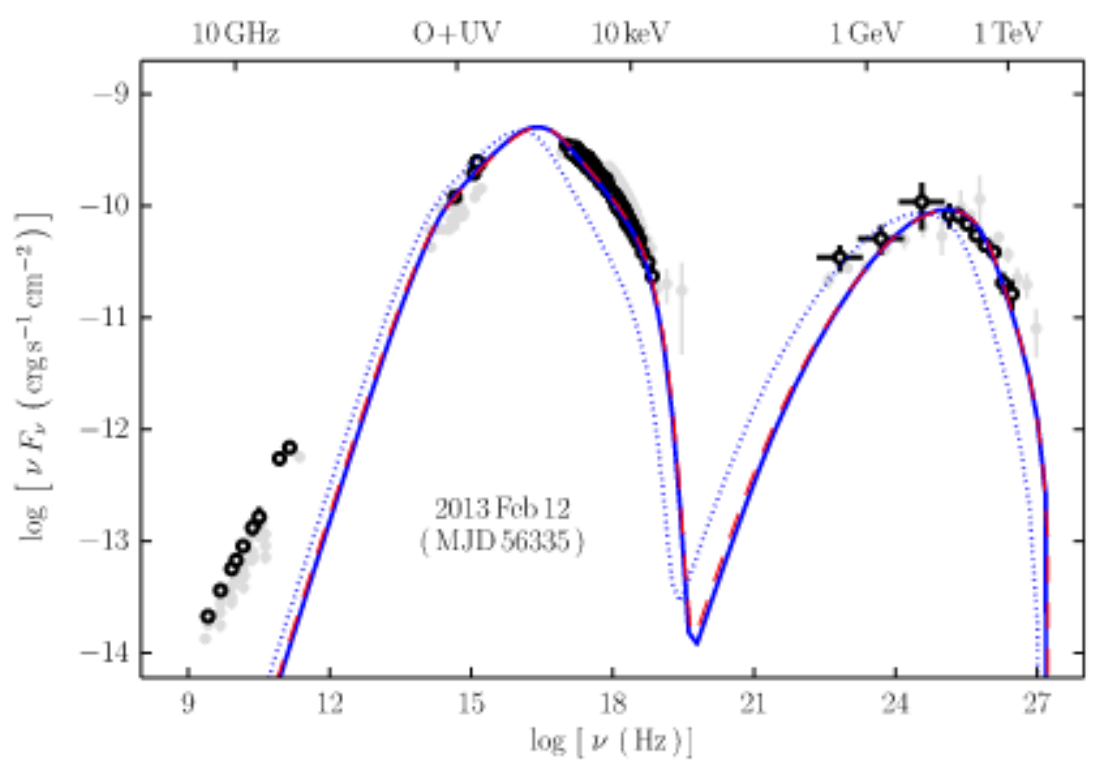

Figure 2.3: Spectral energy distribution of blazar Mrk 421, taken from Baloković et al. (2016). The solid blue line shows a one-zone synchrotron self-Compton (SSC) model based on Böttcher et al. (2013), while the dashed red lines show SED models with timeaveraged electron distribution. The radio data should be considered as upper limits for the SSC model as Mrk 421 is not well-resolved for single-dish radio instruments, so these points include radio emission from scales larger than the jet itself.

sequence" (Cavaliere and D’Elia 2002; Fossati et al. 1998; Ghisellini and Tavecchio 2008; Meyer et al. 2011; Ghisellini et al. 2017) - see Figure 2.4. In the blazar sequence, the most powerful sources displayed relatively small synchrotron peak frequencies, while the least powerful sources had the highest synchrotron peaks. Ghisellini and Tavecchio (2008) explained this as the particles at the most powerful sources having a higher probability of losing energy, which in turn translates into a smaller synchrotron peak. SEDs of flat-spectrum radio quasars (FSRQs) - a subtype of blazar - show the presence of non-thermal radiation emitted in the jet and also thermal radiation from the accretion disc.

Blazars are often subdivided into two categories. Flat-spectrum radio quasars (FSRQs) are characterized by strong, quasar-like emission lines (see Figure 2.5), while BL Lacertae objects (BL Lacs) show weak - or even absent, in some cases - emission lines (see Figure 2.6). In general FSRQs are found at higher redshifts ( $z$ between 1 and 3), while BL Lacs are much closer, with $z \leq 0.5-0.6$ (Ajello et al. 2014). The luminosities 


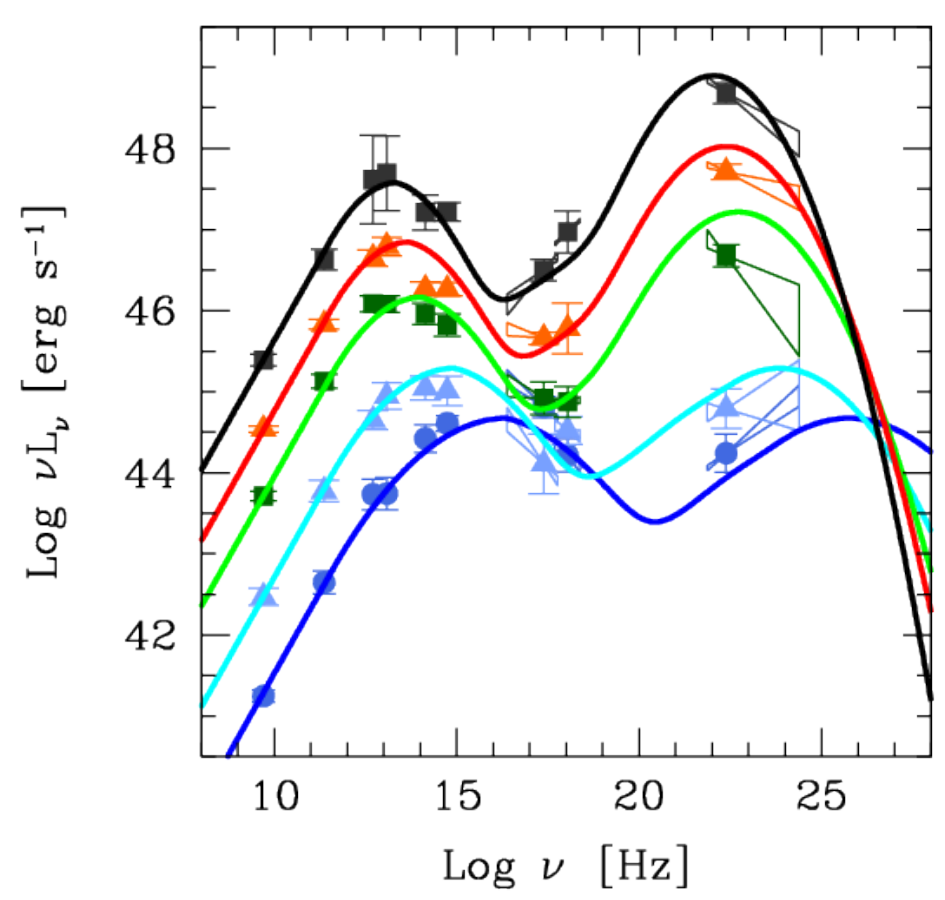

Figure 2.4: The blazar sequence across the electromagnetic spectrum, taken from Ghisellini et al. (2017), adapted from Fossati et al. (1998). From top to bottom: FSRQ, low-energy peaked BL Lac, intermediate-energy peaked BL Lac, high-energy peaked BL Lac, and TeV BL Lac.

and radio structures of blazars, as well as their redshift distributions, suggest they are intrinsically related to radio galaxies, with BL Lacs being associated with FR I galaxies and FSRQs being beamed versions FR II galaxies (Urry and Padovani 1995). 


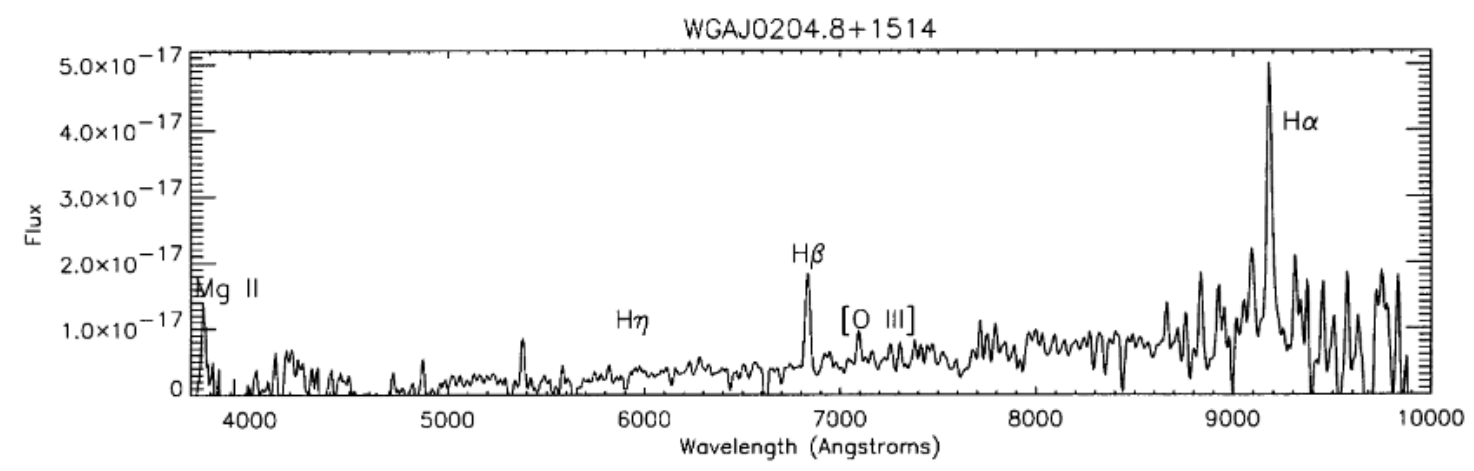

Figure 2.5: Optical spectrum for a FSRQ, taken from Perlman et al. (1998).

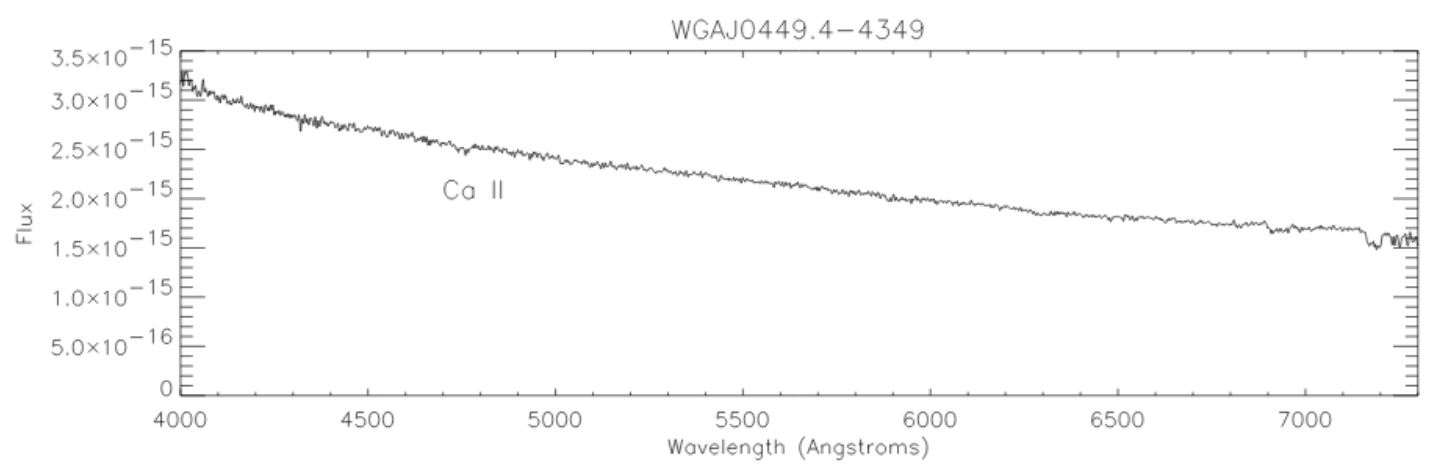

Figure 2.6: Optical spectrum for a BL Lac, taken from Perlman et al. (1998). 


\section{Chapter 3}

\section{Numerical Methods}

\subsection{Introduction}

The equations of General Relativistic Magnetohydrodynamics (GRMHD) govern the motion and dynamics of plasmas flowing in a spacetime described by General Relativity. They appear in a series of astrophysical contexts, including compact objects and jets. Since many of such problems in astrophysics cannot be solved analytically, numerical tools are essential.

Our problems of interest relate to accretion discs and relativistic jets. GRMHD codes have been successfully employed in a comprehensive and distinct range of studies related to black hole accretion. These include theoretical aspects such as the physics of jet launching (Tchekhovskoy, Narayan, and McKinney 2011) and the properties of different types of accretion discs. GRMHD simulations have also shown the emergence of magnetorotational instability - MRI, (Balbus and Hawley 1991; Balbus and Hawley 1998) - as a fundamental process in generating viscosity in discs. Finally, GRMHD codes have also been applied to simulate systems such as Sgr A* (e.g. Mościbrodzka et al. (2009), Mościbrodzka and Falcke (2013), and Mościbrodzka et al. (2014)) and M87 (Mościbrodzka et al. 2017).

HARM - high-accuracy relativistic magnetohydrodynamics - (Gammie, McKinney, and 
Tóth 2003) is a GRMHD code which evolves the equations describing a magnetized plasma living in a background metric $g_{\mu \nu}$. We note that $g_{\mu v}$ is fixed, i.e., not dynamic. Also, since the equations of General Relativity are independent of the coordinate system, we may write the metric in whatever coordinate system is deemed more convenient to the problem at hand. In particular, HARM utilizes a set of modified Kerr-Schild coordinates $\left(x_{0}, x_{1}, x_{2}, x_{3}\right)$ - see McKinney and Gammie (2004) -, which relate to the usual Kerr-Schild coordinates $(t, r, \theta, \phi)$ by

$$
\begin{aligned}
& t=x_{0}, \\
& r=R_{0}+\mathrm{e}^{x_{1}}, \\
& \theta=\pi x_{2}+\frac{1}{2}(1-h) \sin \left(2 \pi x_{2}\right), \\
& \phi=x_{3} .
\end{aligned}
$$

In the above, $R_{0}$ and $h$ (the latter called "hslope" in the code) are parameters that deform the grid appropriately so as to concentrate the grid zones towards the equator, as $h$ decreases, and towards the event horizon, as $R_{0}$ increases.

In GRMHD, we do not include the cases where radiation is dynamically important. Hence, the applicability of HARM and other similar codes is restricted to accretion flows which are optically thin and geometrically thicker, while accreting at lower rates, such as RIAFs. In order to study systems where accretion rates are higher and radiative cooling becomes important, it is necessary to either add a posteriori cooling functions (Shafee et al. 2008; Penna et al. 2010), or to employ radiative GRMHD (GRRMHD) codes, such as KORAL (Sądowski et al. 2013; Sa̧dowski et al. 2014; Chael, Narayan, and Saowski 2017) or bhlight (Ryan, Dolence, and Gammie 2015), which treat the dynamical interactions due to radiation in a more fundamental manner. Thus, the equations that we present here are suitable for studying RIAFs with low accretion rates, but they fail to properly describe Shakura and Sunyaev (1973) thin discs. 
Since the publication of Gammie, McKinney, and Tóth (2003), HARM has undergone a series of upgrades and modifications, having effectively given rise to different codes. These upgrades include 3D adaptations (Noble et al. 2006), an MPI-based version HARMPI, which we use here -, radiative GRMHD codes mentioned above and, more recently, a GPU-accelerated version (Liska et al. 2019). It is often used in conjunction with post-processing codes for radiative transport (Dolence et al. 2009) and/or raytracing (Chan, Psaltis, and Özel 2013; Dexter 2016) and, more recently, with polarization routines (Mościbrodzka and Gammie 2018).

The purpose of this Chapter is twofold: first, we introduce the equations of General Relativistic Magnetohydrodynamics (GRMHD) in the way they are commonly used in numerical codes, such as HARM. A more thorough discussion on these equations, including their derivation, is left to Section 3.4. These equations, which we present in the first part of this chapter, form the backbone of GRMHD codes, as they are the equations that these programs evolve. Our other goal at this stage is to briefly describe the numerical scheme used in HARM, which is done in the second part of this chapter.

\subsection{The equations of GRMHD}

Here, we present the basic equations of GRMHD, highlighting the ones that are evolved in the numerical scheme. A more thorough discussion, including the derivation of the MHD stress-energy tensor in covariant form is left for Section 3.4. In our notation, $c=1$, Greek indices represent the four spacetime coordinates, Latin indices represent the purely spatial components, and repeated indices are summed as per the Einstein summation convention. The Minkowski metric is $\eta_{\mu \nu}=(-1,1,1,1)$.

We start with the conservation of particle number

$$
\nabla_{\mu}\left(n u^{\mu}\right)=0
$$


where $n$ is the particle number density, $u^{\mu}$ is the 4 -velocity and the operator $\nabla_{\mu}$ is the covariant derivative. In component notation, we may write Equation (3.2.1) as

$$
\frac{1}{\sqrt{-g}} \partial_{\mu}\left(\sqrt{-g} \rho u^{\mu}\right)=0
$$

where we have replaced the particle number density $n$ by the rest-mass density $\rho=n m$, $m$ being the particle rest mass, and $g=\operatorname{det}\left(g_{\mu \nu}\right)$.

Next, we write the MHD stress-energy tensor, which we now split into the fluid contribution $T_{\text {fluid }}^{\mu \nu}$ (see, e.g. Landau and Lifshitz 1987) and the electromagnetic contribution $T_{\mathrm{EM}^{\prime}}^{\mu \nu}$

$$
\begin{gathered}
T_{\text {fluid }}^{\mu v}=(\rho+u+p) u^{\mu} u^{v}+p g^{\mu v}, \\
T_{\mathrm{EM}}^{\mu v}=F^{\mu \alpha} F_{\alpha}^{v}-\frac{1}{4} g^{\mu v} F_{\alpha \beta} F^{\alpha \beta},
\end{gathered}
$$

where $u$ is the internal energy, $p$ is the pressure and $F^{\mu v}$ the electromagnetic tensor

$$
F^{\mu v}=\left(\begin{array}{cccc}
0 & E_{x} & E_{y} & E_{z} \\
-E_{x} & 0 & B_{z} & -B_{y} \\
-E_{y} & -B_{z} & 0 & B_{x} \\
-E_{z} & B_{y} & -B_{x} & 0
\end{array}\right) .
$$

The pressure $p$ is related to the internal energy $u$ by

$$
p=(\gamma-1) u
$$

where $\gamma$ is the adiabatic index. In all simulations presented in this work, we assume a fixed value of $\gamma=13 / 9$, which corresponds to relativistic electrons and non-relativistic ions. A few recent studies, such as Ressler et al. (2017), evolve the plasma with different adiabatic indices for the ions and electrons, but our version of the code does not do it. 
We show in Section 3.4 that the electromagnetic part of the stress-energy tensor, Equation (3.2.4), can be written as

$$
T_{\mathrm{EM}}^{\mu v}=b^{2} u^{\mu} u^{v}+\frac{1}{2} b^{2} g^{\mu v}-b^{\mu} b^{v},
$$

where $b^{\mu}$ is the magnetic field 4 -vector, and is related to the three-dimensional magnetic field $B^{i}$ by (Komissarov 1999)

$$
\begin{aligned}
& b^{t}=B^{i} u^{\mu} g_{i \mu}, \\
& b^{i}=\frac{B^{i}+b^{t} u^{i}}{u^{t}} .
\end{aligned}
$$

Using Equation (3.2.7), the MHD stress-energy tensor (see Equations (3.2.3) and (3.2.4)) then becomes

$$
T_{\mathrm{MHD}}^{\mu v}=\left(\rho+u+p+b^{2}\right) u^{\mu} u^{v}+\left(p+\frac{1}{2} b^{2}\right) g^{\mu v}-b^{\mu} b^{v}
$$

The stress-energy tensor Equation (3.2.10) is conserved. This conservation law is expressed as the set of four equations

$$
\nabla_{\mu} T^{\mu}{ }_{v}=0
$$

or, in component notation,

$$
\partial_{t}\left(\sqrt{-g} T^{t}{ }_{v}\right)=-\partial_{i}\left(\sqrt{-g} T^{i}{ }_{v}\right)+\sqrt{-g} T_{\lambda}^{\mathcal{K}} \Gamma_{v \mathcal{K}^{\prime}}^{\lambda}
$$

where $\Gamma_{v \kappa}^{\lambda}$ is the connection coefficient.

Finally, we also have the induction equation, which can be expressed in component 
notation as

$$
\begin{aligned}
\partial_{t}\left(\sqrt{-g} B^{i}\right) & =-\partial_{j}\left[\sqrt{-g}\left(b^{j} u^{i}-b^{i} u^{j}\right)\right], \\
\frac{1}{\sqrt{-g}} \partial_{i}\left(\sqrt{-g} B^{i}\right) & =0 .
\end{aligned}
$$

\subsection{Numerical outline}

The first part of this chapter focused on the physics of GRMHD codes, in particular HARM. There (in conjunction with Section 3.4), our treatment is not meant to be fully rigorous or self-contained, but we still aimed at presenting a logical progression from fluids and electrodynamics to magnetohydrodynamics in a general relativistic setting. Now, we outline the main numerical features of HARM, while the details are left to Section 3.5.

HARM evolves the GRMHD equations using a Godunov-type conservative scheme, meaning that the equations are written in the form

$$
\frac{\partial \mathbf{U}}{\partial t}+\frac{\partial \mathbf{F}(\mathbf{U})}{\partial x^{i}}=0
$$

where $\mathbf{U}$ is a set of conserved variables given by

$$
\mathbf{U}=\sqrt{g}\left(\rho u^{t}, T_{t}^{t}, T_{i}^{t}, B^{i}\right)
$$

The conserved variables are related, $\mathbf{U}(\mathbf{P})$, to a set of primitive variables $\mathbf{P}$ which describe the flow within each grid zone,

$$
\mathbf{P}=\left(\rho, u, v^{i}, B^{i}\right),
$$

where $v^{i}$ is the three-velocity. These are updated at each time step by the fluxes $\mathbf{F}(\mathbf{P})$. 
The evaluation of $\mathbf{F}$ is done with an HLL flux (Harten, Lax, and Leer 1983), which takes into account the fluxes, conserved variables and primitive variables at each side of each grid zone interface. These numerical concepts and tools are presented in Section 3.5 to some level of detail.

To summarize the core calculation performed by the code: we use the primitive variables $v^{i}$ and $B^{i}$ to calculate $u^{t}$ and $b^{\mu}$, using $g_{\mu v} u^{\mu} u^{v}=-1$ and Equations (3.2.8) and (3.2.9). These are then used to calculate the components of the MHD stress-energy tensor, so that both conserved quantities and fluxes may be obtained.

The outflow boundary conditions are implemented by extrapolating the primitive variables from boundary zones into ghost zones ${ }^{1}$ using

$$
P(\text { ghost })=\frac{P(\text { boundary }) \sqrt{-g}(\text { boundary })}{\sqrt{-g}(\text { ghost })} .
$$

We also note that the inner boundary $R_{\text {in }}$ is set to

$$
R_{\text {in }}=0.87 R_{\mathrm{EH}},
$$

where $R_{\mathrm{EH}}$ is the (outer) event horizon, Equation (2.2.3), and the factor 0.87 is an arbitrary choice that allows the inner boundary to be inside the event horizon.

Finally, we note that numerical integration in GRMHD codes allows for negative values of density and internal energies. Since these are unphysical, density and internal energy floors are applied whenever these quantities fall below a threshold value, effectively injecting "artificial" mass and energy.

${ }^{1}$ The ghost zones are the fictitious zones in Section 3.5.3. 


\subsection{The MHD stress-energy tensor in GR}

Here, we arrive at the covariant formulation of the MHD stress-energy tensor. We follow mostly Lichnerowicz (1967) and Anile (1989).

\subsubsection{Covariant formulation of electromagnetism}

Electrodynamics is concerned with the study of charges, currents and the electromagnetic field. The main equations of electrodynamics are Maxwell's equations,

$$
\begin{aligned}
\nabla \cdot \mathbf{E} & =4 \pi \rho, \\
\nabla \cdot \mathbf{B} & =0 \\
\nabla \times \mathbf{E} & =-\frac{1}{c} \frac{\partial \mathbf{B}}{\partial t} \\
\nabla \times \mathbf{B} & =\frac{4 \pi}{c} \mathbf{J}+\frac{1}{c} \frac{\partial \mathbf{E}}{\partial t}
\end{aligned}
$$

where $\mathbf{E}$ is the electric field, $\rho$ is the charge density, $\mathbf{B}$ is the magnetic field and $\mathbf{J}$ is the electric current. We may define the magnetic vector potential $\mathbf{A}$ and the electric potential $\phi$ by

$$
\begin{aligned}
& \mathbf{B}=\nabla \times \mathbf{A}, \\
& \mathbf{E}=-\nabla \phi-\frac{\partial \mathbf{A}}{\partial t} .
\end{aligned}
$$

In the covariant formulation of electrodynamics, we write the four-current as

$$
J^{\mu}=(c \rho, \mathbf{J})
$$

and the four-potential as

$$
A^{\mu}=(\phi / c, \mathbf{A})
$$


The electromagnetic tensor $F_{\mu v}$ is defined as

$$
F_{\mu \nu}=\partial_{\mu} A_{v}-\partial_{v} A_{\mu}
$$

Using Equations (3.4.5), (3.4.6) and (3.4.9), we may write the electromagnetic tensor in matrix form (from now on we set $c=1$ ):

$$
F^{\mu v}=\left(\begin{array}{cccc}
0 & E_{x} & E_{y} & E_{z} \\
-E_{x} & 0 & -B_{z} & B_{y} \\
-E_{y} & B_{z} & 0 & -B_{x} \\
-E_{z} & -B_{y} & B_{x} & 0
\end{array}\right) .
$$

We note that $F^{\mu v}$ is antisymmetric: $F^{\mu v}=-F^{v \mu}$.

The Hodge dual (or simply dual) of an antisymmetric rank-2 tensor $A_{\mu v}$ is defined as

$$
{ }^{*} A_{\mu v} \equiv \frac{1}{2} \varepsilon_{\mu v \alpha \beta} A^{\alpha \beta}
$$

where $\varepsilon_{\mu v \alpha \beta}$ is the Levi-Civita symbol. Applying this to the electromagnetic tensor, Equation (3.4.10), we have

$$
{ }^{*} F^{\mu v}=\left(\begin{array}{cccc}
0 & B_{x} & B_{y} & B_{z} \\
-B_{x} & 0 & -E_{z} & E_{y} \\
-B_{y} & E_{z} & 0 & -E_{x} \\
-B_{z} & -E_{y} & E_{x} & 0
\end{array}\right) .
$$


The covariant versions of Equations (3.4.10) and (3.4.12) are

$$
F_{\mu \nu}=\left(\begin{array}{cccc}
0 & -E_{x} & -E_{y} & -E_{z} \\
E_{x} & 0 & B_{z} & -B_{y} \\
E_{y} & -B_{z} & 0 & B_{x} \\
E_{z} & B_{y} & -B_{x} & 0
\end{array}\right), \quad{ }^{*} F_{\mu \nu}=\left(\begin{array}{cccc}
0 & -B_{x} & -B_{y} & -B_{z} \\
B_{x} & 0 & E_{z} & -E_{y} \\
B_{y} & -E_{z} & 0 & E_{x} \\
B_{z} & E_{y} & -E_{x} & 0
\end{array}\right) .
$$

Using the electromagnetic tensor and the four-current, we may write the Maxwell equations as

$$
\begin{aligned}
\partial_{\alpha} F_{\mu v}+\partial_{v} F_{\alpha \mu}+\partial_{\mu} F_{v \alpha} & =0 \\
\nabla_{v} F^{\mu v} & =4 \pi J^{\mu} .
\end{aligned}
$$

The electromagnetic stress-energy tensor is given by

$$
T_{\mathrm{EM}}^{\mu v}=\frac{1}{4 \pi}\left(F^{\mu \alpha} F_{\alpha}^{v}-\frac{1}{4} g^{\mu v} F_{\alpha \beta} F^{\alpha \beta}\right)
$$

The energy-momentum conservation laws are written as

$$
\nabla_{\alpha} T_{\mathrm{EM}}^{\alpha \beta}=0
$$

\subsubsection{The equations of GRMHD: derivation}

Let $u_{\mu}$ be a comoving velocity, that is, one which is moving with the fluid. Then, $u_{0}=1$ and $u_{i}=0$. Using this and the definition of the electromagnetic tensor Equation (3.4.10) and its dual, we may write

$$
\begin{aligned}
& e_{v}=u^{\mu} F_{\mu v}, \\
& b_{v}=u^{\mu *} F_{\mu v} .
\end{aligned}
$$


Here, we use lower-case letters to identify the four-vectors $e_{\mu}$ and $b_{\mu}$ corresponding to the electric and magnetic fields, respectively. We will use the upper-case $B_{i}$ to refer to the magnetic field three-vector. An immediate consequence of Equations (3.4.18) and (3.4.19) is that

$$
\begin{gathered}
u^{\alpha} e_{\alpha}=u^{\alpha} u^{\mu} F_{\mu \alpha}=-u^{\mu} u^{\alpha} F_{\alpha \mu}=0, \\
u^{\alpha} b_{\alpha}=u^{\alpha} u^{\mu *} F_{\mu \alpha}=-u^{\mu} u^{\alpha *} F_{\alpha \mu}=0,
\end{gathered}
$$

because of the antisymmetry of $F_{\mu \nu}$ and ${ }^{*} F_{\mu \nu}$. Physically, Equations (3.4.20) and (3.4.21) show that the comoving velocity $u^{\mu}$ is orthogonal to the electric and magnetic fields. Using Equations (3.4.20) and (3.4.21), it is possible to construct $F_{\mu \nu}$ and ${ }^{*} F_{\mu \nu}$ in terms of $e_{v}$ and $b_{v}$ :

$$
\begin{gathered}
F_{\mu v}=u_{\mu} e_{v}-u_{v} e_{\mu}+\varepsilon_{\mu v \alpha \beta} u^{\alpha} b^{\beta}, \\
{ }^{*} F_{\mu v}=u_{\mu} b_{v}-u_{v} b_{\mu}+\varepsilon_{\mu \nu \alpha \beta} u^{\alpha} e^{\beta} .
\end{gathered}
$$

The condition of ideal MHD is that in which the electric conductivity goes to infinity. To see how this influences our equations, we start with Ohm's law

$$
\mathbf{j}=\sigma \mathbf{e},
$$

where $\sigma$ is the electric conductivity and $\mathbf{j}$ is the electric current density. If we wish to work in the limit of ideal MHD while keeping a finite conduction current, this implies that we must set $\mathbf{e}=0$ in Equation (3.4.24). Therefore, in the limit of ideal MHD, the electric field for a comoving observer vanishes, and Equation (3.4.18) becomes

$$
u^{\mu} F_{\mu v}=0 .
$$


Our goal here is to construct the stress-energy tensor for ideal MHD as the sum of its fluid and electromagnetic parts,

$$
T_{\mathrm{MHD}}^{\mu v}=T_{\text {fluid }}^{\mu v}+T_{\mathrm{EM}^{\prime}}^{\mu v}
$$

where the fluid part is given by Equation (3.2.3). The electromagnetic part, Equation (3.4.16), will be rewritten using Equation (3.4.22). Since we are working in ideal MHD, the electric field will vanish, so Equation (3.4.22) simplifies to

$$
F_{\mu v}=\varepsilon_{\mu v \alpha \beta} u^{\alpha} b^{\beta}
$$

Thus, we have from Equation (3.4.16),

$$
F^{\mu \alpha} F_{\alpha}^{v}=\left(\varepsilon^{\mu \alpha \lambda \sigma} u_{\lambda} b_{\sigma}\right)\left(\varepsilon^{v}{ }_{\alpha \rho \kappa} u^{\rho} b^{\kappa}\right) .
$$

This can be calculated using

$$
\left(\varepsilon^{\mu \alpha \lambda \sigma} u_{\lambda} b_{\sigma}\right)\left(\varepsilon_{v \alpha \rho \kappa} u^{\rho} b^{\kappa}\right)=-\delta_{v \rho \kappa}^{\mu \lambda \sigma} u_{\lambda} u^{\rho} b_{\sigma} b^{\kappa}
$$

Writing

$$
\begin{aligned}
-\delta_{v \rho \kappa}^{\mu \lambda \sigma} & =-\left[\delta_{v}^{\mu} \delta_{\rho \kappa}^{\lambda \sigma}-\delta_{\rho}^{\mu} \delta_{v \kappa}^{\lambda \sigma}+\delta_{\kappa}^{\mu} \delta_{v \rho}^{\lambda \sigma}\right] \\
& =-\left[\delta_{v}^{\mu}\left(\delta_{\rho}^{\lambda} \delta_{\kappa}^{\sigma}-\delta_{\kappa}^{\lambda} \delta_{\rho}^{\sigma}\right)-\delta_{\rho}^{\mu}\left(\delta_{v}^{\lambda} \delta_{\kappa}^{\sigma}-\delta_{\kappa}^{\lambda} \delta_{v}^{\sigma}\right)+\delta_{\kappa}^{\mu}\left(\delta_{v}^{\lambda} \delta_{\rho}^{\sigma}-\delta_{\rho}^{\lambda} \delta_{v}^{\sigma}\right)\right],
\end{aligned}
$$

and making use of the facts that $u_{\mu} u^{\mu}=-1$ and $u_{\mu} b^{\mu}=0$, we have, for $\mu=v$,

$$
-\delta_{v}^{\mu}\left(\delta_{\rho}^{\lambda} \delta_{\kappa}^{\sigma}-\delta_{\kappa}^{\lambda} \delta_{\rho}^{\sigma}\right) u_{\lambda} u^{\rho} b_{\sigma} b^{\kappa}=\delta_{v}^{\mu} b_{\sigma} b^{\sigma}=\delta_{v}^{\mu} b^{2}
$$


where the reason why we kept $\delta_{v}^{\mu}$ will be clear soon. For $\mu=\rho$,

$$
-\left[-\delta_{\rho}^{\mu}\left(\delta_{v}^{\lambda} \delta_{\mathcal{K}}^{\sigma}-\delta_{\mathcal{K}}^{\lambda} \delta_{v}^{\sigma}\right)\right] u_{\lambda} u^{\rho} b_{\sigma} b^{\kappa}=u_{v} u^{\mu} b_{\sigma} b^{\sigma}=u_{v} u^{\mu} b^{2}
$$

Finally, for $\mu=\kappa$,

$$
-\delta_{\mathcal{K}}^{\mu}\left(\delta_{v}^{\lambda} \delta_{\rho}^{\sigma}-\delta_{\rho}^{\lambda} \delta_{v}^{\sigma}\right) u_{\lambda} u^{\rho} b_{\sigma} b^{\kappa}=-b_{v} b^{\mu}
$$

Using Equations (3.4.30) to (3.4.32), Equation (3.4.29) becomes

$$
\left(\varepsilon^{\mu \alpha \lambda \sigma} u_{\lambda} B_{\sigma}\right)\left(\varepsilon_{v \alpha \rho \kappa} u^{\rho} b^{\kappa}\right)=\delta_{v}^{\mu} b^{2}+u_{v} u^{\mu} b^{2}-b_{v} b^{\mu}
$$

Raising the $v$ index above, we may finally write Equation (3.4.28) as

$$
F^{\mu \alpha} F_{\alpha}^{v}=g^{\mu v} b^{2}+u^{\mu} u^{v} b^{2}-b^{\mu} b^{v}
$$

The second term in Equation (3.4.16) is simpler to rewrite:

$$
\begin{aligned}
F^{\alpha \beta} F_{\alpha \beta} & =\left(\eta^{\alpha \beta \mu v} u_{\mu} b_{v}\right)\left(\eta_{\alpha \beta \rho \kappa} u^{\rho} b^{\kappa}\right) \\
& =-2 \delta_{\rho \kappa}^{\mu v} u_{\mu} u^{\rho} b_{v} b^{\kappa} \\
& =-2\left(\delta_{\rho}^{\mu} \delta_{\kappa}^{v}-\delta_{\kappa}^{\mu} \delta_{\rho}^{v}\right) u_{\mu} u^{\rho} b_{\nu} b^{\kappa} \\
& =2 b^{2} .
\end{aligned}
$$

Hence,

$$
-\frac{1}{4} g^{\mu v} F^{\alpha \beta} F_{\alpha \beta}=-\frac{1}{2} g^{\mu v} b^{2}
$$

After all these calculations, we can now finally write the electromagnetic stress-energy tensor using Equations (3.4.34) and (3.4.35),

$$
T_{\mathrm{EM}}^{\mu v}=b^{2} u^{\mu} u^{v}+\frac{1}{2} b^{2} g^{\mu v}-b^{\mu} b^{v}
$$


where the factor $4 \pi$ has been absorbed into $b_{\mu}$. With Equation (3.2.3) and Equation (3.4.36), we arrive at the stress-energy tensor for MHD:

$$
T_{\mathrm{MHD}}^{\mu v}=\left(\rho+u+p+b^{2}\right) u^{\mu} u^{v}+\left(p+\frac{1}{2} b^{2}\right) g^{\mu v}-b^{\mu} b^{v}
$$

\subsection{Numerical methods}

Here, we go into some detail on the numerical methods employed by HARM. Our goal is to arrive in the HLL flux. To do this, we comment on the Riemann problem, the integral forms of conservation laws and Godunov's method. Our discussion is heavily based on Toro (1999).

\subsubsection{Riemann Problem}

We are interested in the linear advection equation, a partial differential equation given by

$$
\frac{\partial u}{\partial t}+a \frac{\partial u}{\partial x}=0
$$

but now we impose the initial conditions

$$
u(x, 0)=u_{0}(x)= \begin{cases}u_{L} & \text { if } x<0 \\ u_{R} & \text { if } x>0\end{cases}
$$

We note immediately that there is a discontinuity at $x=0$, which will propagate as the system evolves in time. This is the Riemann problem: an initial-value problem given by a conservation equation plus piecewise initial data with a jump discontinuity.

Yet, we expect the points in the initial profile to propagate a distance $d=a t$ within a time $t$, including discontinuities. So, we expect that the characteristic $x=$ at will separate characteristic curves to the left, where the solution is $u_{L}$, from those to the right, where 
the solution is $u_{R}$, that is,

$$
u(x, t)=u_{0}(x-a t)= \begin{cases}u_{L} & \text { if } x-a t<0 \\ u_{R} & \text { if } x-a t>0 .\end{cases}
$$

\subsubsection{Integral Forms of Conservation Laws}

Conservation laws may be written in integral form. To do so, we choose the control volume $V=\left[x_{L}, x_{R}\right] \times\left[t_{1}, t_{2}\right]$. Then, the integral form of the equation for conservation of mass is

$$
\frac{\mathrm{d}}{\mathrm{d} t} \int_{x_{L}}^{x_{R}} \rho(x, t) \mathrm{d} x=f\left(x_{L}, t\right)-f\left(x_{R}, t\right)
$$

where $f=\rho v, \rho$ is the mass density and $v$ the velocity. For a complete system, we have instead of Equation (3.5.4)

$$
\frac{\mathrm{d}}{\mathrm{d} t} \int_{x_{L}}^{x_{R}} \mathbf{U}(x, t) \mathrm{d} x=\mathbf{F}\left(\mathbf{U}\left(x_{L}, t\right)\right)-\mathbf{F}\left(\mathbf{U}\left(x_{R}, t\right)\right),
$$

whose corresponding differential form is

$$
\frac{\partial \mathbf{U}}{\partial t}+\frac{\partial \mathbf{F}(\mathbf{U})}{\partial x}=0
$$

If we integrate Equation (3.5.5) in time between $t_{1}$ and $t_{2}$, with $t_{1} \leq t_{2}$, we arrive at

$$
\int_{x_{L}}^{x_{R}} \mathbf{U}\left(x, t_{2}\right) \mathrm{d} x=\int_{x_{L}}^{x_{R}} \mathbf{U}\left(x, t_{1}\right) \mathrm{d} x+\int_{t_{1}}^{t_{2}} \mathbf{F}\left(\mathbf{U}\left(x_{L}, t\right)\right) \mathrm{d} t-\int_{t_{1}}^{t_{2}} \mathbf{F}\left(\mathbf{U}\left(x_{R}, t\right)\right) \mathrm{d} t,
$$

which is the integral form of conservation laws.

\subsubsection{Discretization and Conservative Methods}

The numerical methods used for solving partial differential equations replace the continuous problem described by these PDEs by a finite set of discrete values, which may 
be obtained upon a discretization of the domain of the PDEs into a finite set of points or volumes via a mesh or a grid. Thus, the corresponding discretization of the PDEs on such a grid results in discrete values. These discrete values may be of two types:

- Finite Difference approach: they are regarded as point values defined at grid points;

- Finite Volume approach: they are regarded as averages over finite volumes.

HARM uses a Finite Volume approach.

\section{Godunov's Method}

The method proposed by Godunov is a conservative scheme in which the intercell numerical fluxes $f_{i+1 / 2}$ are computed by using solutions of local Riemann problems, that is, the Riemann problems at each cell interface. It is assumed that at any given time $n$ the data has a piecewise constant distribution. The data may be seen as pairs of constant states $\left(u_{i}^{n}, u_{i+1}^{n}\right)$ separated by a discontinuity at the intercell boundary $x_{i+1 / 2}$. Thus, a Riemann problem arises naturally. It is defined by

$$
\begin{gathered}
\frac{\partial u}{\partial t}+\frac{\partial f(u)}{\partial x}=0, \\
u(x, 0)=u_{0}(x)= \begin{cases}u_{i}^{n}, & \text { if } x<0, \\
u_{i+1}^{n}, & \text { if } x>0 .\end{cases}
\end{gathered}
$$

We say then that we have a local Riemann problem $R P\left(u_{i}^{n}, u_{i+1}^{n}\right)$ with initial data $\left(u_{i}^{n}, u_{i+1}^{n}\right)$ and our task is to find a solution to the global Riemann problem at a later time, $n+1$.

We may update a cell value $u_{i}^{n}$ to a new values $u_{i}^{n+1}$ using the following scheme proposed by Godunov. We solve two Riemann problems, $R P\left(u_{i-1}^{n}, u_{i}^{n}\right)$ and $R P\left(u_{i}^{n}, u_{i+1}^{n}\right)$, hereafter $R P_{-}$and $R P_{+}$, then take an integral average in the cell $i$ of the combined solutions of these two local problems, and assign this value to $u_{i}^{n+1}$. 
To illustrate, suppose we have a linear advection equation, whose flux is $f=a u$, with $a>0$. The exact solutions for the two Riemann problems $R P_{-}$and $R P_{+}$are, respectively,

$$
u_{i-1 / 2}(x / t)= \begin{cases}u_{i-1 / 2}^{n}, & \text { if } x / t<a \\ u_{i}^{n}, & \text { if } x / t>a\end{cases}
$$

and

$$
u_{i+1 / 2}(x / t)= \begin{cases}u_{i}^{n}, & \text { if } x / t<a \\ u_{i+1}^{n}, & \text { if } x / t>a .\end{cases}
$$

The solution proposed by Godunov's scheme is defined by

$$
u_{i}^{n+1}=\frac{1}{\Delta x}\left[\int_{0}^{\frac{1}{2} \Delta x} u_{i-1 / 2}(x / \Delta t) \mathrm{d} x+\int_{-\frac{1}{2} \Delta x}^{0} u_{i-1 / 2}(x / \Delta t) \mathrm{d} x\right]
$$

The solution Equation (3.5.11) may be written as

$$
u_{i}^{n+1}=\frac{1}{\Delta x} \int_{x_{i-1 / 2}}^{x_{i+1 / 2}} \tilde{u}(x, t) \mathrm{d} x
$$

where $\tilde{u}(x, t)$ is understood as the combined solution of $\left(R P_{-}, R P_{+}\right)$, both of which are exact solutions. Thus, $\tilde{u}(x, t)$ is an exact solution.

Now, we write the integral form of $\tilde{u}(x, t)$ in the control volume $\left[x_{i-1 / 2}, x_{i+1 / 2}\right] \times$ $[0, \Delta t]$

$$
\int_{x_{i-1 / 2}}^{x_{i+1 / 2}} \tilde{u}(x, \Delta t) \mathrm{d} x=\int_{x_{i-1 / 2}}^{x_{i+1 / 2}} \tilde{u}(x, 0) \mathrm{d} x+\int_{0}^{\Delta t} f\left(\tilde{u}\left(x_{i-1 / 2}, t\right)\right) \mathrm{d} t-\int_{0}^{\Delta t} f\left(\tilde{u}\left(x_{i+1 / 2}, t\right)\right) \mathrm{d} t .
$$

Defining the intercell fluxes as time integral averages

$$
f_{i \mp 1 / 2}=\frac{1}{\Delta t} \int_{0}^{\Delta t} f\left(\tilde{u}\left(x_{i \mp 1 / 2}, t\right)\right) \mathrm{d} t
$$

and using the definition Equation (3.5.12), we note that Equation (3.5.13) naturally takes 
the form of a conservation law,

$$
u_{i}^{n+1}=u_{i}^{n}+\frac{\Delta t}{\Delta x}\left[f_{i-1 / 2}-f_{i+1 / 2}\right]
$$

The integrand $f(\tilde{u}(x, t))$ at each cell interface depends on the exact solution $\tilde{u}(x, t)$ of the Riemann problems along the $t$-axis. It is given by $\tilde{u}\left(x_{1 \mp 1 / 2, t}\right)=u_{i \mp 1 / 2}(0)$, so that the intercell fluxes become $f_{i \mp 1 / 2}=f\left(u_{i \mp 1 / 2}(0)\right)$.

The generalization to a multidimensional case is straightforward, with Equation (3.5.15) becoming

$$
\mathbf{U}_{i}^{n+1}=\mathbf{U}_{i}^{n}+\frac{\Delta t}{\Delta x}\left[\mathbf{F}_{i-1 / 2}-\mathbf{F}_{i+1 / 2}\right]
$$

where $\mathbf{F}_{i \mp 1 / 2}=\mathbf{F}\left(\mathbf{U}_{i \mp 1 / 2}(0)\right)$.

\section{Boundary Conditions}

The conservative formula can be applied to all cells except for the first and last, cells 1 and $M$, since in these cases there is only one intercell flux. There are then two possibilities for boundary conditions:

- We can define boundary functions $u_{l}(t)$ and $u_{r}(t)$ so the flux at the boundaries are $f_{1 / 2}\left(u_{l}(t)\right)$ and $f_{M+1 / 2}\left(u_{r}(t)\right)$;

- We can use fictitious cells, also called ghost cells, to the left of the innermost domain cell and to the right of the outermost cell with cell averages $u_{0}^{n}$ and $u_{M+1}^{n}$ (values obtained upon extrapolation from boundary cells are a common method, as well), so we can solve Riemann problems at these interfaces.

The choice between boundary functions and fictitious cells depends on the physics of the problem. HARM utilizes ghost cells. 


\subsubsection{The HLL Riemann Solver}

In practice, the Riemann problem is solved countless times during a calculation, which makes the Riemann problem solution process an extremely demanding task in the numerical method. Therefore, it is useful to find approximations to solve the Riemann problem that make all the numerical processes involved somewhat of a less demanding task.

The HLL solver (after Harten, Lax and van Leer) provides an approximation for the intercell numerical fluxes. The central idea is to assume a wave configuration for the solution that consists of two waves separating three constant states. Assuming that the wave speeds are given by some algorithm, we apply the integral form of the conservation law to arrive at a closed-form, approximate expression for the flux.

Suppose we have the control volume $\left[x_{l}, x_{R}\right] \times[0, T]$. The whole wave structure arising from the exact solution of the Riemann problem is contained in this control volume, at a time $T$, i.e., $x_{L} \leq T S_{L}$ and $x_{R} \geq T S_{R}$, where $S_{L}, S_{R}$ are the fastest signal velocities perturbing the initial data $U_{L}, U_{R}$. The integral form of the conservation law is

$$
\begin{aligned}
\int_{x_{L}}^{x_{R}} \mathbf{U}(x, T) \mathrm{d} x & =\int_{x_{L}}^{x_{R}} \mathbf{U}(x, 0) \mathrm{d} x+\int_{0}^{T} \mathbf{F}\left(\mathbf{U}\left(x_{L}, t\right)\right) \mathrm{d} t-\int_{0}^{T} \mathbf{F}\left(\mathbf{U}\left(x_{R}, t\right)\right) \mathrm{d} t \\
& =x_{R} \mathbf{U}_{R}-x_{L} \mathbf{U}_{L}+T\left(\mathbf{F}_{L}-\mathbf{F}_{R}\right),
\end{aligned}
$$

where $\mathbf{F}_{L}=\mathbf{F}\left(\mathbf{U}_{\mathbf{L}}\right)$ and $\mathbf{F}_{R}=\mathbf{F}\left(\mathbf{U}_{\mathbf{R}}\right)$. The left-hand side of Equation (3.5.17) can be written as

$$
\begin{aligned}
\int_{x_{L}}^{x_{R}} \mathbf{U}(x, T) \mathrm{d} x & =\int_{x_{L}}^{T S_{L}} \mathbf{U}(x, T) \mathrm{d} x+\int_{T S_{L}}^{T S_{R}} \mathbf{U}(x, T) \mathrm{d} x+\int_{T S_{R}}^{x_{R}} \mathbf{U}(x, T) \mathrm{d} x \\
& =\left(T S_{L}-x_{L}\right) \mathbf{U}_{L}+\int_{T S_{L}}^{T S_{R}} \mathbf{U}(x, T) \mathrm{d} x-\left(x_{R}-T S_{R}\right) \mathbf{U}_{R}
\end{aligned}
$$


Equating Equations (3.5.17) and (3.5.18), we obtain

$$
\int_{T S_{L}}^{T S_{R}} \mathbf{U}(x, T) \mathrm{d} x=T\left(S_{R} \mathbf{U}_{R}-S_{L} \mathbf{U}_{L}+\mathbf{F}_{L}-\mathbf{F}_{R}\right)
$$

that is,

$$
\frac{1}{T\left(S_{R}-S_{L}\right)} \int_{T S_{L}}^{T S_{R}} \mathbf{U}(x, T) \mathrm{d} x=\mathbf{U}^{\text {hll }},
$$

where

$$
\mathbf{U}^{\text {hll }} \equiv \frac{S_{R} \mathbf{U}_{R}-S_{L} \mathbf{U}_{L}+\mathbf{F}_{L}-\mathbf{F}_{R}}{S_{R}-S_{L}}
$$

This is the average of the exact solution of the Riemann problem between $x_{L}$ and $x_{R}$. Now, we apply the conservation law for the control volume $\left[x_{L}, 0\right] \times[0, T]$ :

$$
\int_{T S_{L}}^{0} \mathbf{U}(x, T) \mathrm{d} x=-T S_{L} \mathbf{U}_{L}+T\left(\mathbf{F}_{L}-\mathbf{F}_{0 L}\right)
$$

where $\mathbf{F}_{0 L}$ is the flux along the $t$-axis. From Equation (3.5.22) we have

$$
\mathbf{F}_{0 L}=\mathbf{F}_{L}-S_{L} \mathbf{U}_{L}-\frac{1}{T} \int_{T S_{L}}^{0} \mathbf{U}(x, T) \mathrm{d} x
$$

Analogously, for the control volume $\left[0, x_{R}\right] \times[0, T]$,

$$
\mathbf{F}_{0 R}=\mathbf{F}_{R}-S_{R} \mathbf{U}_{R}+\frac{1}{T} \int_{0}^{T S_{R}} \mathbf{U}(x, T) \mathrm{d} x .
$$

So far, all relations obtained and used are exact, because we assumed the exact solutions of the RP. Harten, Lax and van Leer proposed an approximation which consists of three regions (constant states) separated by two waves, that is, all intermediate states are dumped into Equation (3.5.21). More precisely, assuming that $S_{L}$ and $S_{R}$ are known, 
HLL proposed that we replace the exact solution $\mathbf{U}(x, t)$ by

$$
\tilde{\mathbf{U}}(x, t)=\left\{\begin{array}{lr}
\mathbf{U}_{L} & \text { if } x / t \leq S_{L}, \\
\mathbf{U}^{\text {hll }} & \text { if } S_{L} \leq x / t \leq S_{R}, \\
\mathbf{U}_{R} & \text { if } x / t \geq S_{R} .
\end{array}\right.
$$

Using $\tilde{\mathbf{U}}(x, t)$ defined above, we get the flux in the middle region given by Equation (3.5.23) or Equation (3.5.24)

$$
\begin{aligned}
& \mathbf{F}^{\text {hll }}=\mathbf{F}_{L}+S_{L}\left(\mathbf{U}^{\text {hll }}-\mathbf{U}_{L}\right), \\
& \mathbf{F}^{\text {hll }}=\mathbf{F}_{R}+S_{R}\left(\mathbf{U}^{\text {hll }}-\mathbf{U}_{R}\right) .
\end{aligned}
$$

Substituting the definition of $\mathbf{U}^{\text {hll }}$ Equation (3.5.21) in either Equations (3.5.26) and (3.5.27) above, we arrive at

$$
\mathbf{F}^{\mathrm{hll}}=\frac{S_{R} \mathbf{F}_{L}-S_{L} \mathbf{F}_{R}+S_{L} S_{R}\left(\mathbf{U}_{R}-\mathbf{U}_{L}\right)}{S_{R}-S_{L}}
$$

Therefore, the intercell flux will be given by

$$
\mathbf{F}_{i+1 / 2}^{\text {hll }}=\left\{\begin{array}{lr}
\mathbf{F}_{L} & \text { if } 0 \leq S_{L}, \\
\mathbf{F}^{\text {hll }} & \text { if } S_{L} \leq 0 \leq S_{R} \\
\mathbf{F}_{R} & \text { if } 0 \geq S_{R} .
\end{array}\right.
$$




\section{Chapter 4}

\section{Jet efficiencies and black hole spins in}

\section{jetted quasars}

The work presented in this chapter has been accepted for publication in MNRAS.

\section{Summary of this Chapter}

The mechanisms responsible for the production of relativistic jets from supermassive black holes (SMBHs) accreting at near-Eddington rates are not well-understood. Simple theoretical expectations indicate that SMBHs in quasars accrete via thin discs which should produce at most very weak jets. This is contradicted by observations of powerful jets in flat-spectrum radio quasars (FSRQs). We use gamma-ray luminosities observed with the Fermi Large Area Telescope as a proxy of the jet power for a population of 154 FSRQs. Assuming typical quasar accretion rates and using black hole mass measurements from a variety of methods, we find a mean jet production efficiency of about $10 \%$ for FSRQs, with values as high as $222 \%$. We find that this is consistent with FSRQs hosting moderately thin, magnetically arrested accretion s around rapidly spinning black holes (BHs). Modeling our observations using general relativistic 
54CHAPTER 4. JET EFFICIENCIES AND BLACK HOLE SPINS IN JETTED QUASARS radiative magnetohydrodynamic simulations of jets from thin discs, we find an average lower limit of $a_{*}=0.59$ for the SMBH spins of FSRQs, with tendency for the spins to decrease as the black hole mass increases. Our results are consistent with the merger-driven evolution of SMBHs. 3\% of the sample cannot be explained by current GRRMHD models of jet production from Kerr BHs due to the high efficiencies. Along the way, we find a correlation between BH masses and $L_{\gamma}$ which may be an useful mass estimator of blazar gamma-ray studies. 


\subsection{Introduction}

Blazars are among the most powerful active galactic nuclei (AGN), producing relativistic jets roughly aligned with the line of sight of the observer. As a result, they become strongly Doppler boosted, thereby allowing the jet emission to dominate the spectrum of blazars. Based on their observational properties, it is customary to divide blazars into two different categories: flat-spectrum radio quasars (FSRQs) are characterised by significant broad emission lines observed in their spectra, while blazars with barely any noticeable emission lines are called BL Lacertae (BL Lac) objects (Urry and Padovani 1995; Massaro, Thompson, and Ferrara 2016).

The extended radio structure and large radio luminosities of FSRQs suggest that Fanaroff-Riley Type II - FR II (Fanaroff and Riley 1974) - radio galaxies are the parent populations of radio-loud quasars, whereas FR I galaxies are the parent populations of BL Lacs (Urry and Padovani 1995). In view of this, Böttcher and Dermer (2002) and Cavaliere and D'Elia (2002) proposed evolutionary models of blazars, concluding that FSRQs would evolve into BL Lacs as their accretion decreases over time, which is supported by the findings in Ajello et al. (2014).

FSRQs are conventionally interpreted as supermassive black holes (SMBHs) accreting at high mass accretion rates $\dot{M} \gtrsim 0.01 \dot{M}_{\text {Edd }}$ and are therefore surrounded by thin accretion discs $\left(\dot{M}_{\text {Edd }}\right.$ is the Eddington accretion rate); BL Lacs have $\dot{M} \lesssim 0.01 \dot{M}_{\text {Edd }}$, hence their SMBHs are fed via radiatively inefficient accretion flows (RIAFs) - e.g. Ghisellini, Maraschi, and Tavecchio (2009) and Ghisellini et al. (2014).

According to standard models, the formation of jets of relativistic jets is a function of mainly two parameters: the mass accretion rate in units of the Eddington rate $\dot{m} \equiv$ $\dot{M} / \dot{M}_{\mathrm{Edd}}$ and the dimensionless black hole $(\mathrm{BH})$ spin $a_{*} \equiv a / M$ (Contopoulos, Gabuzda, and Kylafis 2014). For instance, the Blandford-Znajek (BZ) mechanism (Blandford and Znajek 1977)) implies that it is possible to extract rotational energy of Kerr BHs threaded 
by large-scale magnetic field lines which are brought to their vicinity by the accreted gas. Therefore, the magnetic field — in practice represented by the magnetic flux $\phi$ at the event horizon — is another fundamental parameter to be considered when studying jet formation (Semenov, Dyadechkin, and Punsly 2004).

FSRQs and FR II radio galaxies produce bright, powerful jets and should have standard, geometrically thin, optically thick accretion discs (Ghisellini and Celotti 2001; Jester 2005; Ghisellini, Maraschi, and Tavecchio 2009). Therefore, observationally it is clear that stronger jets associated with FSRQs can be produced by thin discs, contrary to some theoretical expectations (Meier 2001; Nemmen et al. 2007). General relativistic magnetohydrodynamic (GRMHD) simulations are now part of the standard machinery of tools used to study the dynamics and electromagnetic appearance of accreting BHs (Porth et al. 2019). GRMHD models have been advancing tremendously due to a combination of algorithmic advances and Moore's law. The state of the art in terms of jet formation models in high- $\dot{M}$ BH systems involve the addition of radiation pressure effects in the numerical models: general relativistic radiative MHD - GRRMHD (Sa̧dowski et al. 2014; McKinney et al. 2014; Ryan, Dolence, and Gammie 2015). Such models are beginning to reach a level where they can make testable predictions. Therefore, it is worth assessing the state of the numerical models when confronted with observations of jetted quasars, and what constraints on the model parameters can be obtained from such comparisons.

General relativistic magnetohydrodynamic (GRMHD) simulations are commonly used to study accreting black holes (BHs) and the systems' behavior under different values of the parameters, such as $\mathrm{BH}$ spin and magnetic field configuration. In particular, "magnetically arrested discs" (MAD, (Narayan, Igumenshchev, and Abramowicz 2003; Tchekhovskoy, Narayan, and McKinney 2011; McKinney, Tchekhovskoy, and Blandford 2012)) have been shown to launch powerful jets. In the MAD scenario, magnetic flux accumulates in the innermost region of the disc, and is prevented from 
escaping due to the pressure exerted by the gas being accreted. At the same time, the accumulated magnetic flux breaks down the accretion flow into streams and blobs, therefore effectively disrupting the accretion process onto the black hole. If jets are launched by the BZ process, the extracted power $P_{\mathrm{BZ}}$ can be expressed as (Blandford and Znajek 1977; Tchekhovskoy, Narayan, and McKinney 2010)

$$
P_{\mathrm{BZ}}=\frac{\kappa}{4 \pi c} \Omega_{\mathrm{H}}^{2} \Phi_{\mathrm{BH}}^{2} f\left(\Omega_{\mathrm{H}}\right),
$$

where $\Phi_{\mathrm{BH}}$ is the magnetic flux threading the black hole, and $\Omega_{\mathrm{H}}$ is the angular frequency of the $\mathrm{BH}$ horizon. Hence, given that FSRQs produce extremely powerful jets originating from black holes accreting via RIAFs (see, for instance, G15), MADs naturally emerge as an explanation.

Ghisellini et al. (2014) found that a strong correlation between jet power and accretion luminosity with the jet power dominating the disc luminosities, in agreement with MAD expectations. Zamaninasab et al. (2014) found in a sample dominated by FSRQs that the estimated values of $\phi$ are consistent with MAD predictions. The MAD hypothesis was questioned by van Velzen and Falcke (2013), who studied a sample of FR II quasars and found a jet production efficiency $\eta \approx 0.01$ - considerably lower than MAD predictions and other observational estimates (Ghisellini et al. 2014; Zamaninasab et al. 2014; Zdziarski et al. 2015).

Many GR(R)MHD works have investigated thin discs and their energy outflows (Shafee et al. 2008; Noble, Krolik, and Hawley 2009; Noble, Krolik, and Hawley 2010; Penna et al. 2010; Noble et al. 2011; Avara, McKinney, and Reynolds 2016; Liska et al. 2019). In particular, Avara, McKinney, and Reynolds (2016) performed a systematic numerical investigation of the radiative and jet efficiencies of thin MADs around Kerr BHs as a function of $a_{*}$. Besides finding that thin MADs achieve a radiative efficiency of $15 \%$ which is twice the Novikov-Thorne value, they fitted a function to jet production 
efficiency resulting from several models and proposed a function $\eta=\eta\left(a_{*}, \phi, h\right)$ which is related to the jet power as $P=\eta\left(a_{*}, \Phi\right) \dot{M} c^{2}$ and $h$ is the disc thickness $h \equiv H / R$ where $H$ is the scale height. The advantage of this model and the simplicity of the fitting function for $\eta$ is that one can quickly check whether the typical jet efficiencies predicted by GRMHD MAD models can explain observations of quasars, as well as performing quantitative constraints on the $\mathrm{BH}$ parameters such as the spin.

Indeed, the Avara et al. model was applied to observations of FR II quasars by Rusinek et al. (2016), who found that the jets in their sample could only be explained by the MAD scenario if the discs are thicker than predicted in standard theory. Inoue et al. (2017) analyzed a large sample of more than 7000 radio-loud quasars detected at 1.4 GHz and SDSS optical spectra and found jet efficiencies comparable to those suggested by van Velzen and Falcke (2013), and a low average spin of $a_{*}=0.13$, which is smaller than cosmological merger SMBH evolution models (Volonteri, Sikora, and Lasota 2007; Volonteri et al. 2013).

Previous works such as Rusinek et al. (2016) and Inoue et al. (2017) have based their samples on radio and optical observations of radio-loud quasars. FSRQs are bright gamma-ray emitters and are behind the majority of point sources observed by the gamma-ray instruments. For instance, the fermi-Large Area Telescope (LAT) has observed more than 600 FSRQs in the $0.1-300 \mathrm{GeV}$ energy range (Acero et al. 2015; The Fermi-LAT Collaboration 2019). Here, we will use Fermi-LAT observations of FSRQs which constrain their energetics-concretely, their jet powers and jet production efficiencies-with the goal of assessing how well current models for jet formation from high- $\dot{M}$ BHs are able to explain such observations. Along the way, we will obtain quantitative estimates of $\mathrm{BH}$ spins for jetted quasars, which may shed light on the spin evolution of SMBHs.

For all our cosmological calculations in this Chapter, we use $H_{0}=67.74 \mathrm{~km} \mathrm{Mpc}^{-1} \mathrm{~s}^{-1}$, $\Omega_{\mathrm{m}_{0}}=0.31$, and $\Omega_{\Lambda_{0}}=0.69$ (Planck Collaboration et al. 2016). 


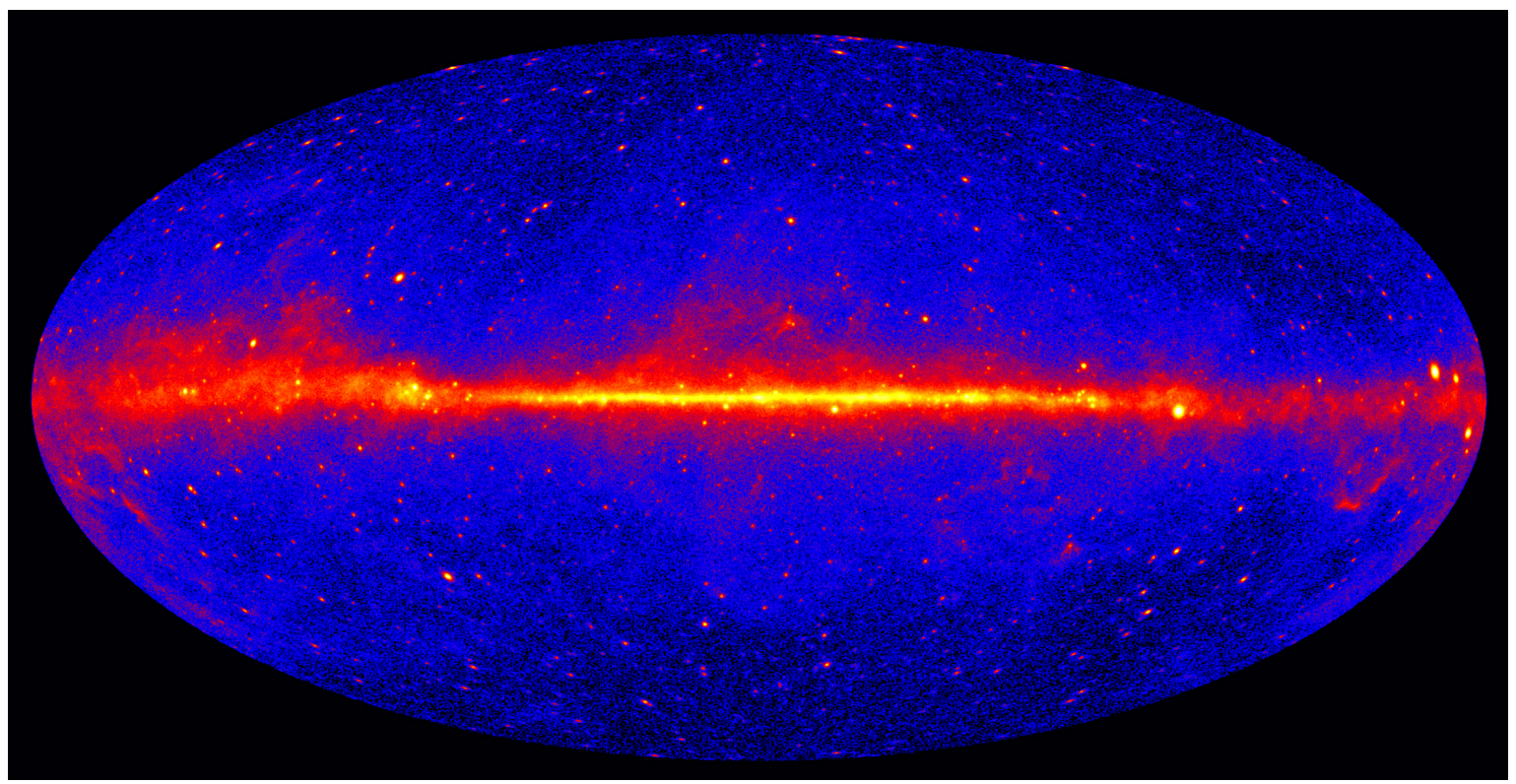

Figure 4.1: The $\gamma$-ray sky: energies greater than $1 \mathrm{GeV}$ as detected on the first five years of data from Fermi-LAT. Source: NASA/DOE/Fermi LAT Collaboration.

\subsection{The Fermi Gamma-ray Space Telescope}

Launched in 2008, Fermi is a $\gamma$-ray space telescope observing from low Earth orbit. Its Large Area Telescope (LAT) instrument performs a complete scan of the sky at every 3 hours, with a peak effective area of about $1 \mathrm{~m}^{2}$ and a field of view of 2.4 sr at $1 \mathrm{GeV}$, LAT's highest sensitivity (Atwood et al. 2009). A pair-production and conversion telescope, LAT detects incoming photons within an energy range varying between $20 \mathrm{MeV}$ and $300 \mathrm{GeV}$. These interact with silicon strips and produce electronpositron pairs, $\gamma \rightarrow e^{-}+e^{+}$, which are tracked as they pass through the strips until they reach a cesium iodide calorimeter, where their energies are then measured. Figure 4.1, comprising 5 years of observations, shows the $\gamma$-ray sky at energies greater than $1 \mathrm{GeV}$ as detected by Fermi-LAT.

Fermi's instruments LAT and Gamma-ray Burst Monitor (GBM) are associated with many important results and discoveries. These include the Fermi bubbles towering over the Milky Way (Su, Slatyer, and Finkbeiner 2010; Ackermann et al. 2014b), $\gamma$-ray variability in PG 1553+113 (Ackermann et al. 2015), the extremely bright gamma-ray burst GRB 130427A (Ackermann et al. 2014a), flares in 3C 279 (Abdo et al. 2010; Paliya 
2015; Ackermann et al. 2016) and, more recently, the short gamma-ray burst associated with the merger of two neutron stars (Abbott et al. 2017a). An extensive review focused on extragalactic $\gamma$-ray sources and Fermi results associated with them can be found in Massaro, Thompson, and Ferrara (2016).

Despite being strongest in $\gamma$-rays, blazar multiwavelength emission allows for combined studies which paint a more complete picture of the blazar scenario. These have implications in both observational features and purely theoretical descriptions of phenomena associated with these sources.

For instance, correlations between radio and $\gamma$-ray luminosities were studied in, among others, Ackermann et al. (2011), León-Tavares et al. (2012), and Massaro et al. (2013). Combined IR and $\gamma$-ray data showed a clear distinction between $\gamma$-ray emitting blazars other AGN classes, thus allowing for an association between $\gamma$-ray sources and their counterparts at lower energies (D'Abrusco et al. 2014).

Associations between $\gamma$-ray features with those at other wavelengths also extend to optical and X-rays. In Ghisellini and Tavecchio (2015), optical emission lines were used to infer the disc luminosity and compare them with $\gamma$-ray jet luminosities, while other studies have shown that many blazars present in Fermi catalogs are also found in X-ray surveys - see, for instance, Sambruna et al. (2010) -, despite X-ray luminosity being faint due to it being between the two large humps in blazar SEDs.

Fermi observations have also been useful in providing a clear distinction between BL Lacs and FSRQs, with a $\gamma$-ray luminosity divide at roughly $10^{46-47} \mathrm{erg} \mathrm{s}^{-1}$ (Ghisellini, Maraschi, and Tavecchio 2009). This has been interpreted as accretion occurring at different rates in these two classes, with as BL Lacs being fed by radiatively inefficient accretion flows leading to weak ionizing fluxes and therefore to weak or absent emission lines. FSRQs, on the other hand, would prefer radiatively efficient thin discs. 


\subsection{Data selection}

For our study, we need a sample of jetted quasars with estimated jet powers and BH masses. This sample was provided by Ghisellini et al. (2014) and Ghisellini and Tavecchio (2015) (hereafter G14 and G15, respectively).

G14 and G15 published a sample of blazars that have been detected in $\gamma$-rays by Fermi-LAT and spectroscopically observed in the optical band (Shaw et al. 2012; Shaw et al. 2013), including 229 FSRQs and 475 BL Lacs. The Ghisellini sample is based on the first and second Fermi-LAT catalogs, corresponding to only two years of $\gamma$-ray observations. We have now more than ten years of LAT observations, therefore we cross-matched the original sample of FSRQs from G14 and G15 with the most up-todate catalog of LAT sources - 4FGL; The Fermi-LAT Collaboration (2019) - ending up with 191 FSRQs.

We now describe our procedure for matching the FSRQs to the 4FGL catalog. Since different catalogs adopt different naming conventions for their objects and report slightly different declinations and right ascensions, matching the FSRQs in G15 with the FSRQs detected by Fermi 4FGL using some kind of direct comparison between the object names, their aliases in either catalog or their position in the sky is not very effective. To overcome this, we employed a "distance method". For each object in G15, we compared its coordinates with those of the objects in Fermi 4FGL, then calculated the separation $d$ given by

$$
d=\sqrt{\left[\left(\alpha_{\mathrm{G}}-\alpha_{\mathrm{F}}\right) \cos \left(\delta_{F}\right) \cos \left(\delta_{G}\right)\right]^{2}+\left(\delta_{\mathrm{G}}-\delta_{\mathrm{F}}\right)^{2}}
$$

between this object and each object in Fermi 4FGL. We then selected the smallest distance, $d_{\mathrm{min}}$, and compared it to a threshold distance $d_{\mathrm{thr}}$. If $d_{\min } \leq d_{\mathrm{thr}}$, we concluded that the objects match. In Eq. 4.3.1, the subindices F and G refer to the Fermi 4FGL and the G15 objects, respectively, the declination $\delta$ and right ascension $\alpha$ are given in 
radians. After some trial and error, we settled on a threshold value $d_{\text {thr }}$ corresponding to one minute. Hence, $d \leq 1$ minute implied a match between the objects.

Using this method, we were able to identify 156 objects in the Fermi 4FGL catalog, out of the 191 sources in G15. Still, the objects $1438+3710$ and $1439+3712$ in G15 were both associated with 4FGL J1438.9+3710 by our distance method. Similarly, the objects $1636+4715$ and $1637+4717$ were both associated with 4FGL J1637.7+4717. Given their significantly different redshifts, we searched the literature and the 4FGL and G15 aliases to determine which object should be correctly associated with both 4FGL sources, and we determined that both $1439+3712$ and $1636+4715$ should be excluded from our analysis. Hence, our final sample contains 154 objects. Table 4.3 lists the FSRQs names, coordinates along with the other relevant properties for this work such as their $\gamma$-ray luminosities and $\mathrm{BH}$ masses.

The 37 remaining objects from G15 which remained unmatched with 4FGL objects could possibly be due to sources which were bright enough for a few months to be included in previous Fermi LAT catalogs but not over the entire 8-year period comprised by Fermi 4FGL (Paliya et al. 2017). Furthermore, at least one object in G15 appears listed as BL Lac elsewhere. Therefore, we chose to stick to our "distance method" based on Equation (4.3.1) to select our sample, and the non-identification of the 37 remaining objects does not compromise the data or the results presented in this work. The redshifts of the FSRQs considered here vary between 0.226 and 3, with a mean value of 1.31 (Figure 4.2 ).

G15 estimated the BH masses using four different methods. Three of these are virial estimates based on emission lines measured by Shaw et al. (2012), whereas the other uses a disc-fitting method based on the Shakura and Sunyaev (1973) thin disc model. In this work, the values we use for the masses are obtained upon averaging the virial estimates of G15. We ignore their disc-fitting estimates altogether because this method could not be applied to all FSRQs in the original sample of G15 due to poor data or 


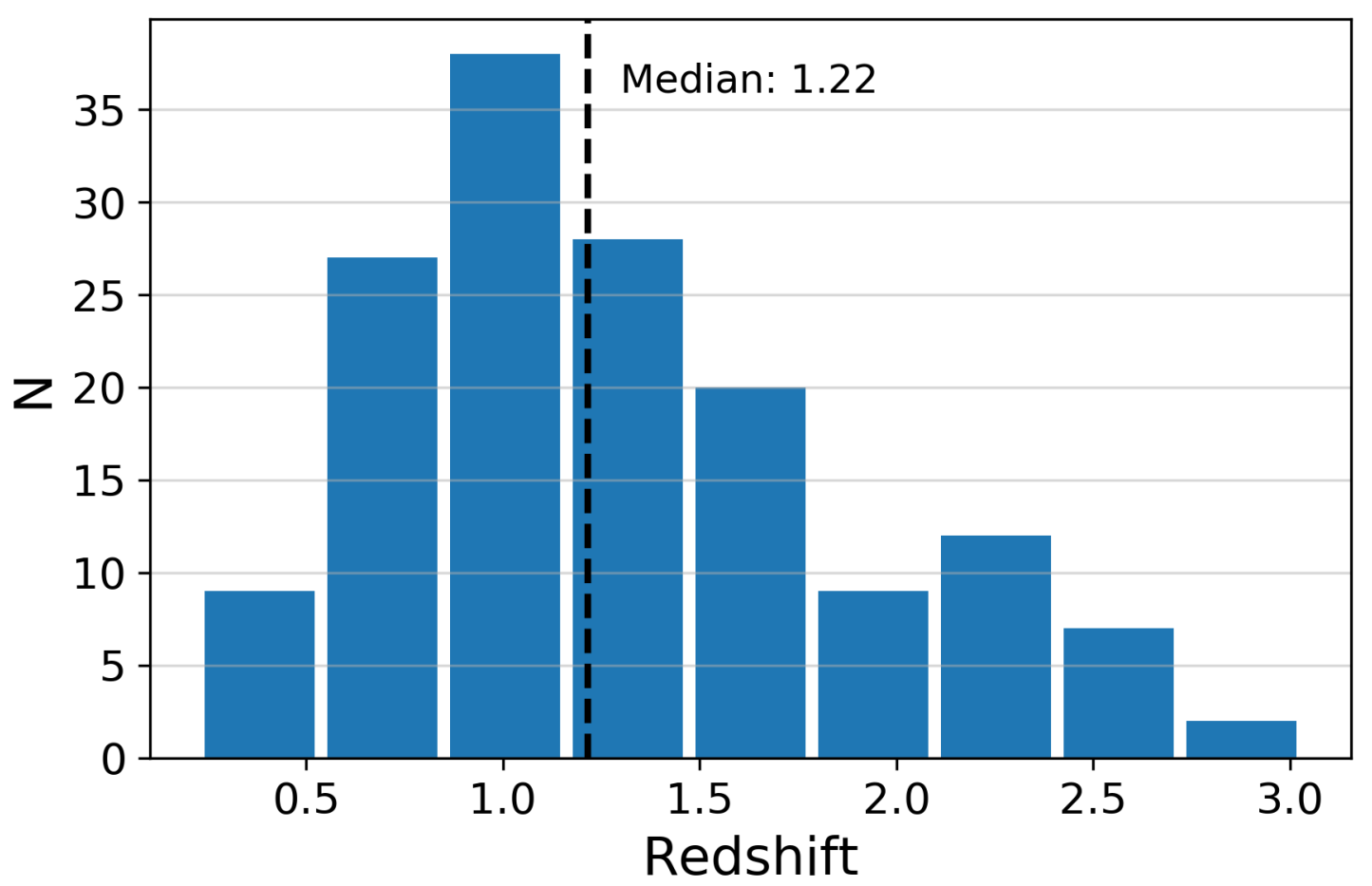

Figure 4.2: Redshift distribution of the 154 FSRQs in our sample.

overdominance of the synchrotron jet component (Ghisellini and Tavecchio 2015). The uncertainty of the mass measurements obtained by the virial method is 0.5 dex in all emission lines. Figure 4.3 displays the mass distribution of the objects in our sample.

The gamma-ray luminosities $L_{\gamma}$ observed with Fermi-LAT are shown in Figure 4.4. They were estimated from the 4 FGL energy fluxes $F_{\gamma}$ in the $100 \mathrm{MeV}$ to $100 \mathrm{GeV}$ range obtained assuming a power-law model:

$$
L_{\gamma}=4 \pi d_{L}^{2} \frac{F_{\gamma}}{(1+z)^{\left(1-\alpha_{g}\right)}}
$$

where $d_{L}$ is the luminosity distance $\left(H_{0}=67.74 \mathrm{~km} \mathrm{Mpc}^{-1} \mathrm{~s}^{-1}\right.$; Planck Collaboration et al. (2016)) and $\alpha_{g}=1-n$ is the energy spectral index, with $n$ being the power-law spectral index. 


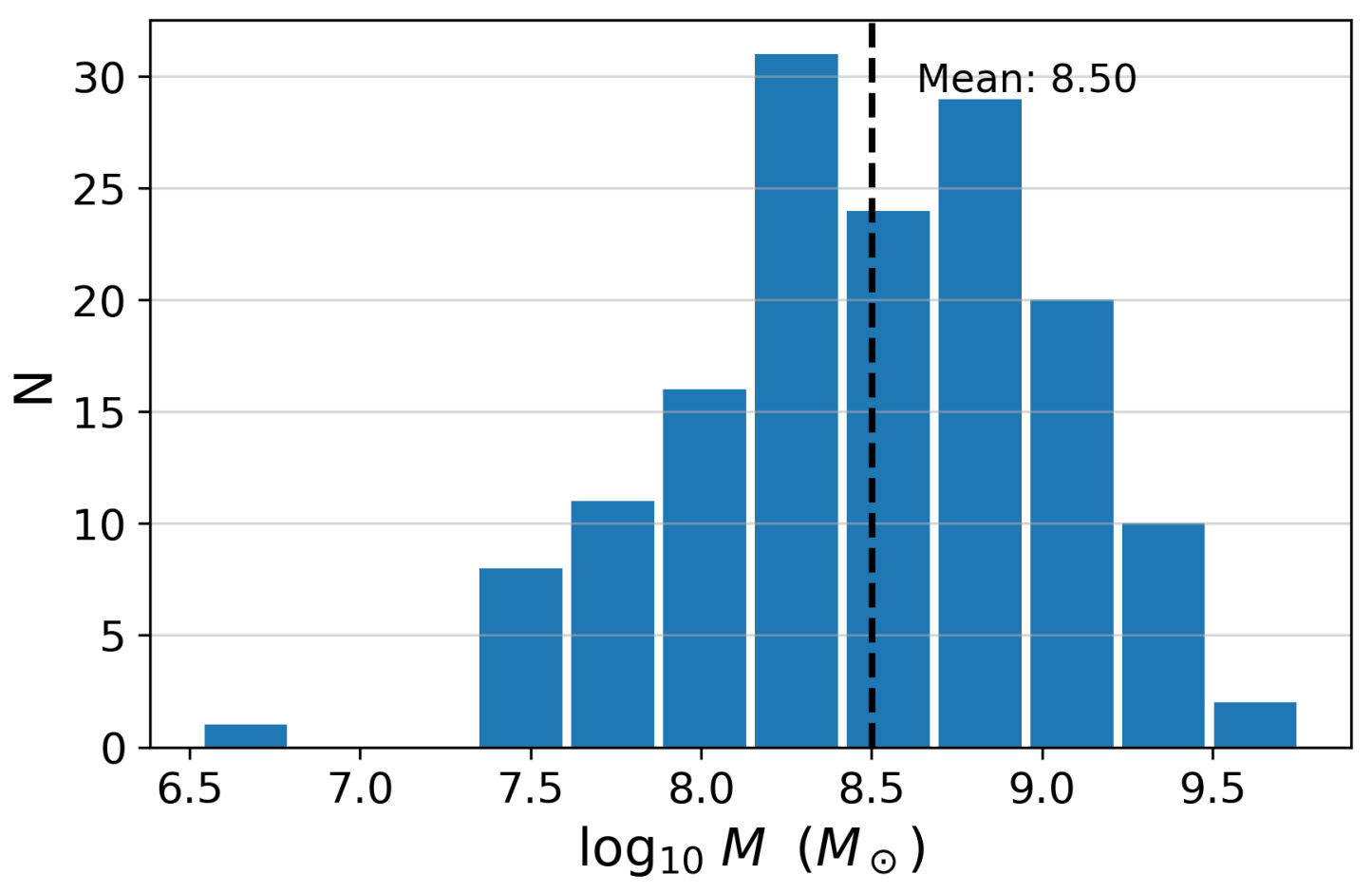

Figure 4.3: Distribution of the black hole masses $M$ in our sample.

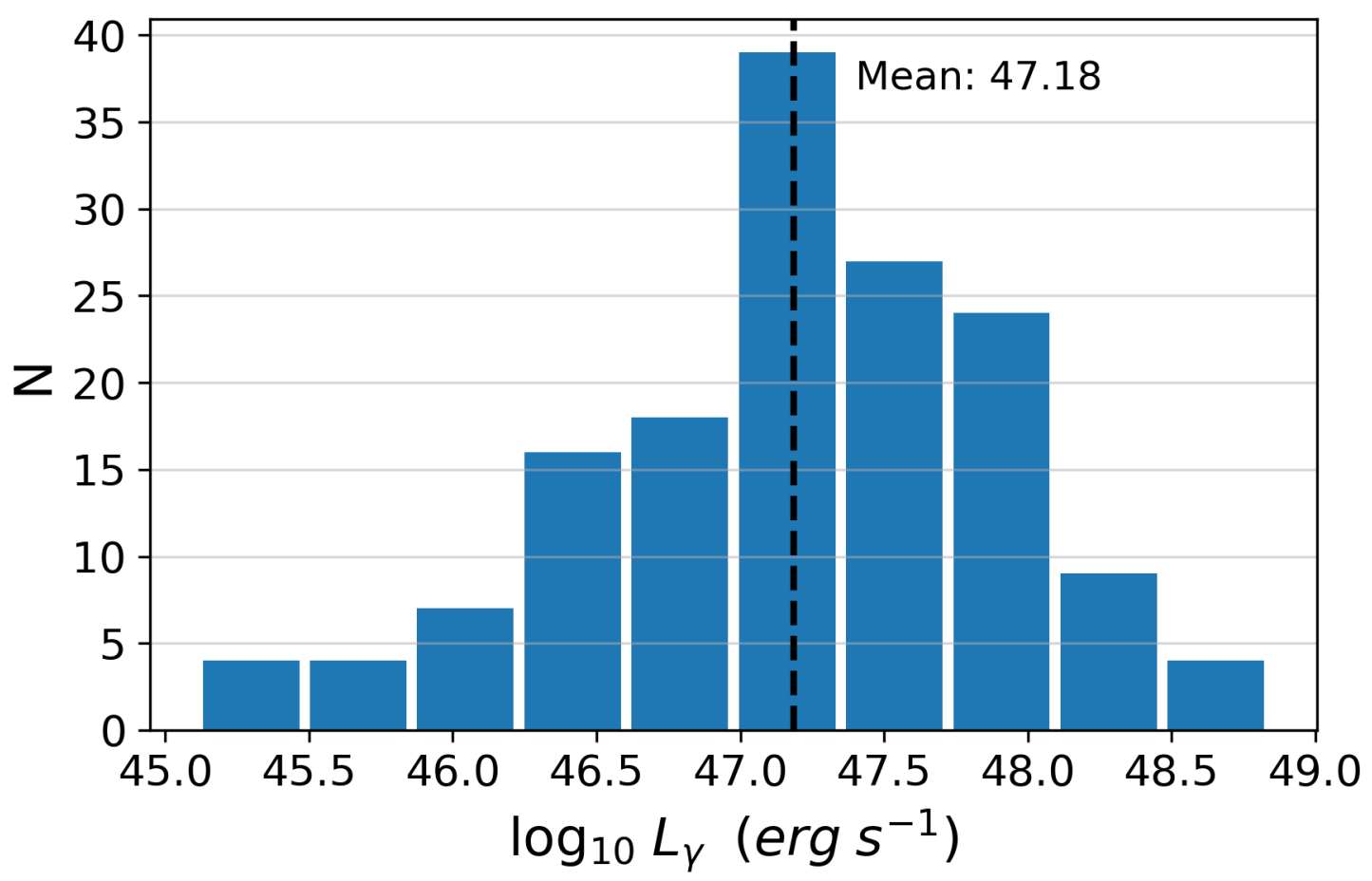

Figure 4.4: Distribution of the gamma-ray luminosities in our sample. 


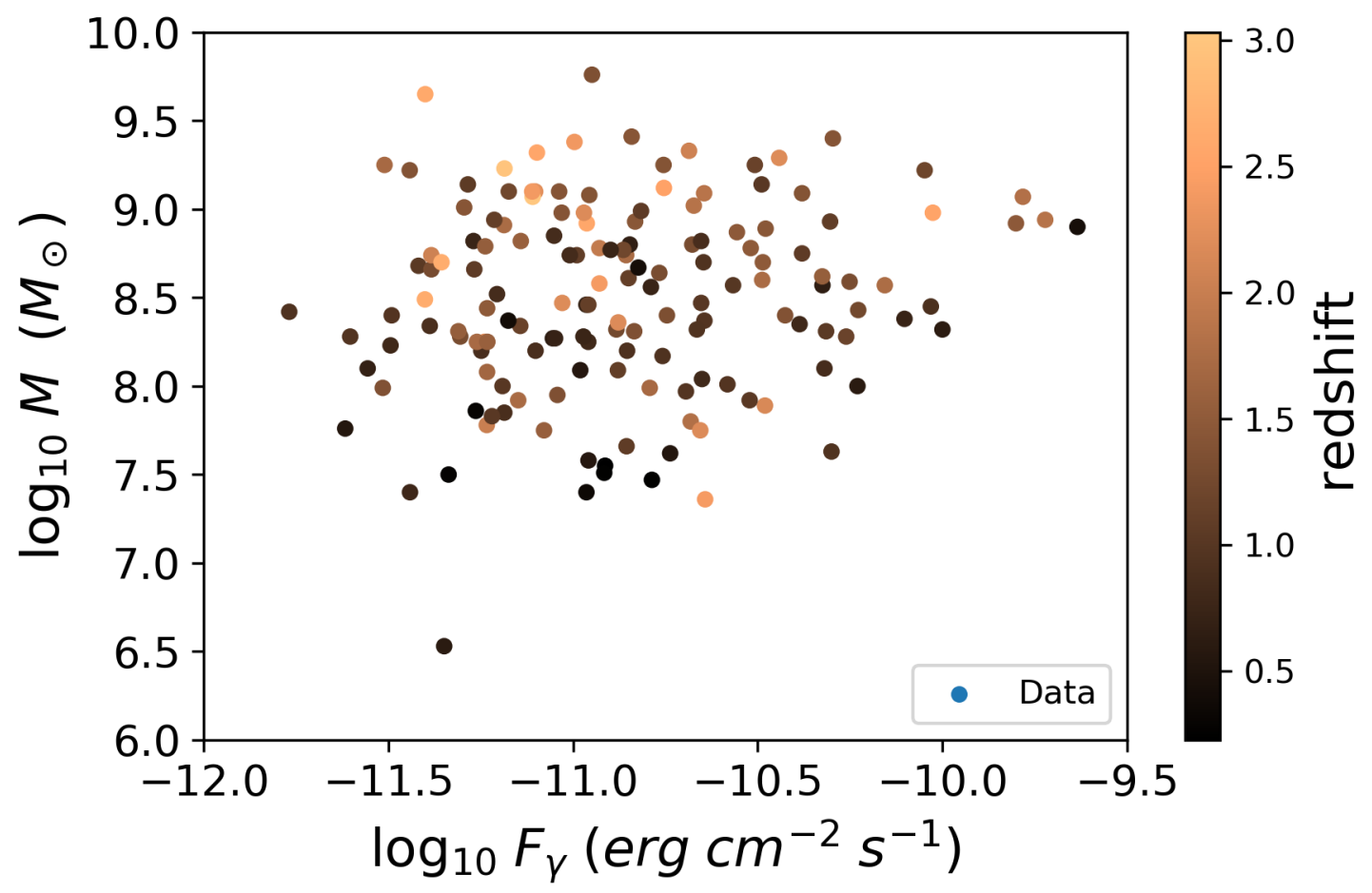

Figure 4.5: Black hole mass estimates collected from G15 plotted against the Fermi-LAT gamma-ray flux.

\subsection{Results}

Figure 4.5 shows the gamma-ray flux plotted against the black hole masses for all objects in our sample. We find only a weak level of correlation between $F_{\gamma}$ and $M$ : the Pearson correlation coefficient is $r=0.15$ resulting in a probability of no-correlation of $p_{\text {null }}=0.07$ (two-tailed $p$-value) —a high chance of the result being consistent with the null hypothesis.

Figure 4.6 shows the gamma-ray luminosity as a function of the black hole mass and redshift $z$ for all objects in our sample. On average, the FSRQs with higher gamma-ray luminosities tend to be located at higher redshifts - e.g. Ajello et al. (2014). Figure 4.6 suggests a possible correlation between the $\mathrm{BH}$ mass and $L_{\gamma}$. Indeed, the Pearson correlation coefficient is $r=0.5$ resulting in a probability of no-correlation of $p_{\text {null }}=10^{-11}$.

Given the above value of $p_{\text {null, }}$ it is tempting to conclude that there is a strong 


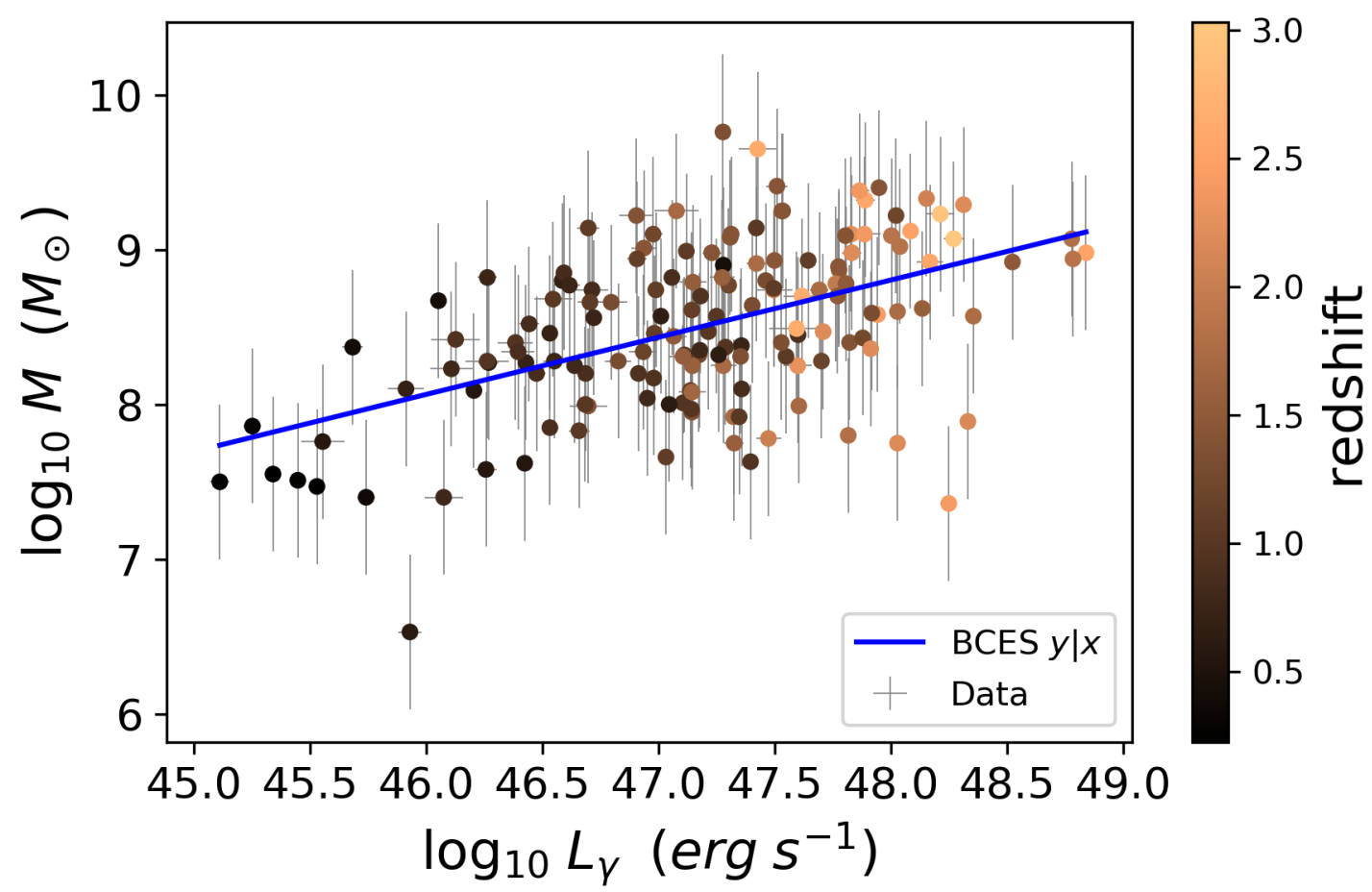

Figure 4.6: Black hole mass plotted against the Fermi-LAT gamma-ray luminosity. The uncertainties in $L_{\gamma}$ are too small and barely visible, while the uncertainties in the black hole mass are much larger. The solid blue line corresponds to our best-fit line.

correlation between these two variables. This would be puzzling given the weak correlation between the flux and $M$ in Figure 4.5. To settle the issue, we performed a partial correlation analysis of the common dependence of $L \gamma$ and $M$ on $d_{L}$ using the partial Kendall's tau correlation test (Akritas and Siebert 1996). Considering the log of these values, we find that the p-value of the null hypothesis (i.e. no correlation between $L \gamma$ and $M$ in the presence of the third variable $d_{L}$ ) is very low (Table 4.1). Therefore, the $L_{\gamma}-M$ correlation is unlikely to be entirely a distance-driven artifact.The fact that $M$ is moderately correlated with $z\left(r=0.43, p_{\text {null }}=10^{-8}\right)$, combined with $L_{\gamma} \propto d_{L}^{-2}$ explains why the $M-L_{\gamma}$ correlation is stronger than the $M$-Flux one: Figure 4.6 is showing two variables that depend on the distance to different degrees.

We fitted a linear relation

$$
\log _{10} M=A \log _{10} L_{\gamma}+B
$$


Table 4.1: Results of partial correlation analysis. Columns (1)-(2): Quantities (Z is $\log _{10} d_{\mathrm{L}}$ in both cases). Column (3): Subsample; Column (4): Number of sources; Columns (5)-(7): results of partial correlation analysis; $\tau$ is the partial Kendall's correlation coefficient; $\sigma$ is the square root of the calculated variance; $P_{\text {null }}$ is the probability for accepting the null hypothesis that there is no correlation between $\mathrm{X}$ and Y; Column (7) gives the associated significance in standard deviations with which the null hypothesis is rejected.

\begin{tabular}{ccccccc}
\hline $\mathrm{X}$ & $\mathrm{Y}$ & Objects & $\mathrm{N}$ & $\tau$ & $\sigma$ & $P_{\text {null }}$ \\
\hline $\log _{10} L_{\gamma}$ & $\log _{10} M$ & all & 150 & 0.21 & 0.04 & $5 \times 10^{-7}(5 \sigma)$ \\
\hline
\end{tabular}

to our data using the BCES $y \mid x$ method (Akritas and Bershady 1996) with $M$ in solar masses and $L_{\gamma}$ in erg s ${ }^{-1}$. The BCES $y \mid x$ method is a regression procedure that takes into account uncertainties in both variables and assumes that $M$ is the dependent variablesomething justifiable in this case given the much larger uncertainties affecting $M$. The best-fit parameters resulting from the fit are $A=0.37 \pm 0.05$ and $B=-8.95 \pm 2.42$. The corresponding fit is indicated in Figure 4.6. The scatter about the best-fit is 0.5 dex. This correlation between $\mathrm{BH}$ mass and $L_{\gamma}$ may be an useful mass estimator in blazar $\gamma$-ray studies in the absence of other, more conventional mass proxies such as broad emission lines.

Nemmen et al. (2012) obtained a tight correlation between $L_{\gamma}$ and the total jet power of blazars:

$$
\log _{10} P_{\text {jet }}=(0.51 \pm 0.02) \log _{10} L_{\gamma}+(21.2 \pm 1.1)
$$

with both variables in units of $\mathrm{erg} \mathrm{s}^{-1}$. Here, we use this correlation to directly estimate the jet power from the measured values of $L_{\gamma}$. In Nemmen et al. (2012), the scatter around the best-fit is 0.5 dex, which we take as the uncertainty in our $P_{\text {jet }}$ estimates. The resulting mean of $\log _{10} P_{\text {jet }}$ is 45.26 .

In order to estimate the efficiency of jet production $\eta=P_{\text {jet }} /\left(\dot{M} c^{2}\right)$, we need mass accretion rates for the quasars in the our sample. An excellent assumption (McLure and Dunlop 2004; Ghisellini et al. 2010) is to simply assume that the SMBHs in quasars are accreting at a level of 10 per cent the Eddington rate, i.e. take $\dot{M}=0.1 \dot{M}_{\mathrm{Edd}}=L_{\mathrm{Edd}} / c^{2}$ 


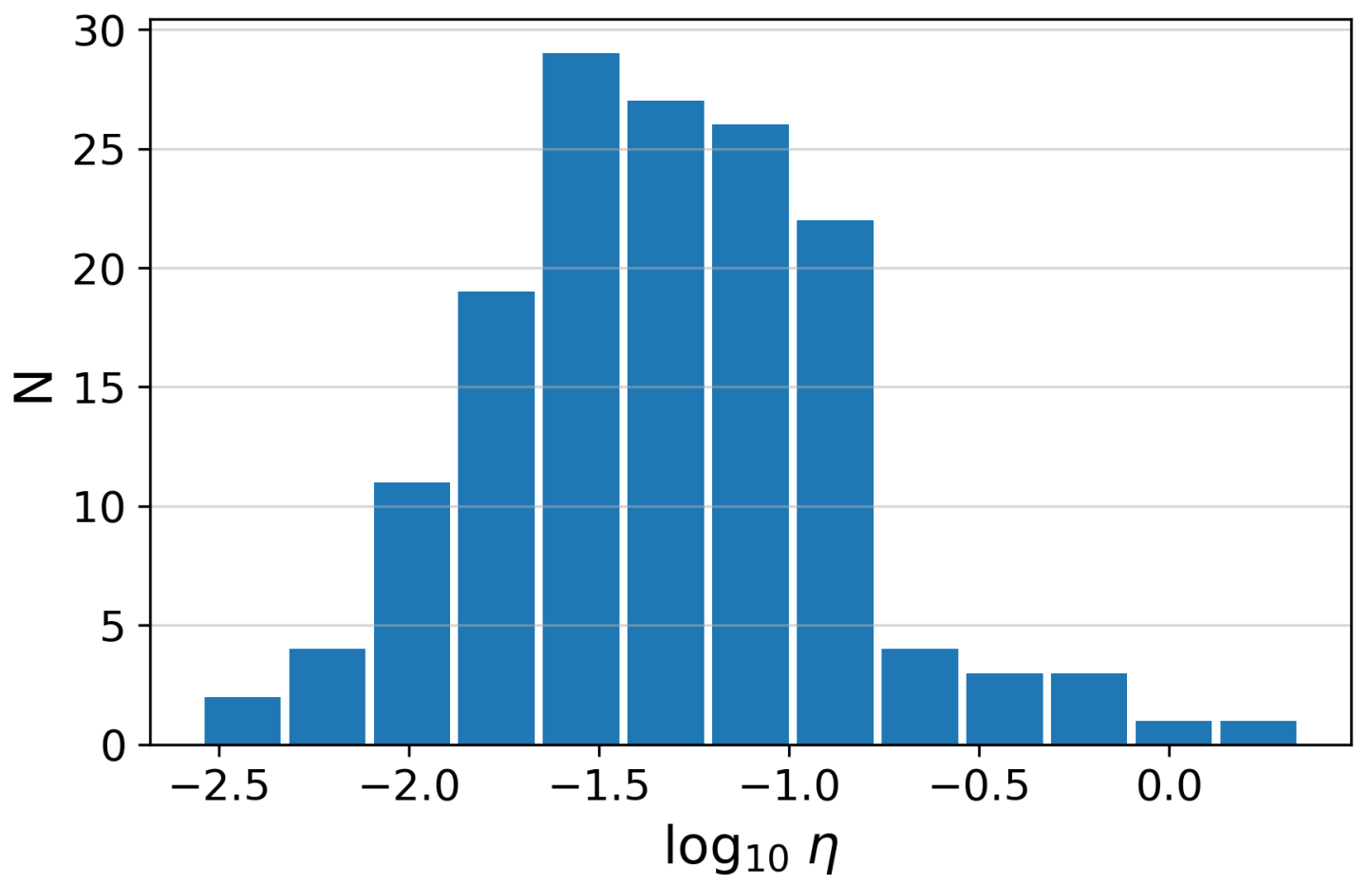

Figure 4.7: Distribution of the jet production efficiencies for the FSRQs in our sample assuming that the SMBHs are accreting at $10 \%$ of the Eddington rate.

where $L_{\mathrm{Edd}}$ is the Eddington luminosity.

The distribution of observationally-constrained jet production efficiencies for the objects in our sample is plotted in Figure 4.7. We note that the 0.5 dex uncertainties in both $P_{\text {jet }}$ and $M$ imply large uncertainties in $\eta$ of about $0.7 \mathrm{dex}$. The median efficiency in our sample is 0.04 ; the minimum and maximum efficiencies are, respectively, about $3 \times 10^{-3}$ and 2.

Figure 4.8 displays the relation between $\eta$ and the $\mathrm{BH}$ mass. By construction, $\eta \propto M^{-1}$ due to our assumption of $\dot{M} \propto \dot{M}_{\mathrm{Edd}}$. Therefore, one must be careful in interpreting any possible correlations between $\eta$ and $M$. Given this important caveat and keeping in mind the considerable uncertainties affecting our estimates of $\eta$, we find an anticorrelation between $\eta$ and $M$,

$$
\eta=0.1\left(\frac{M}{10^{8} M_{\odot}}\right)^{-0.64} .
$$




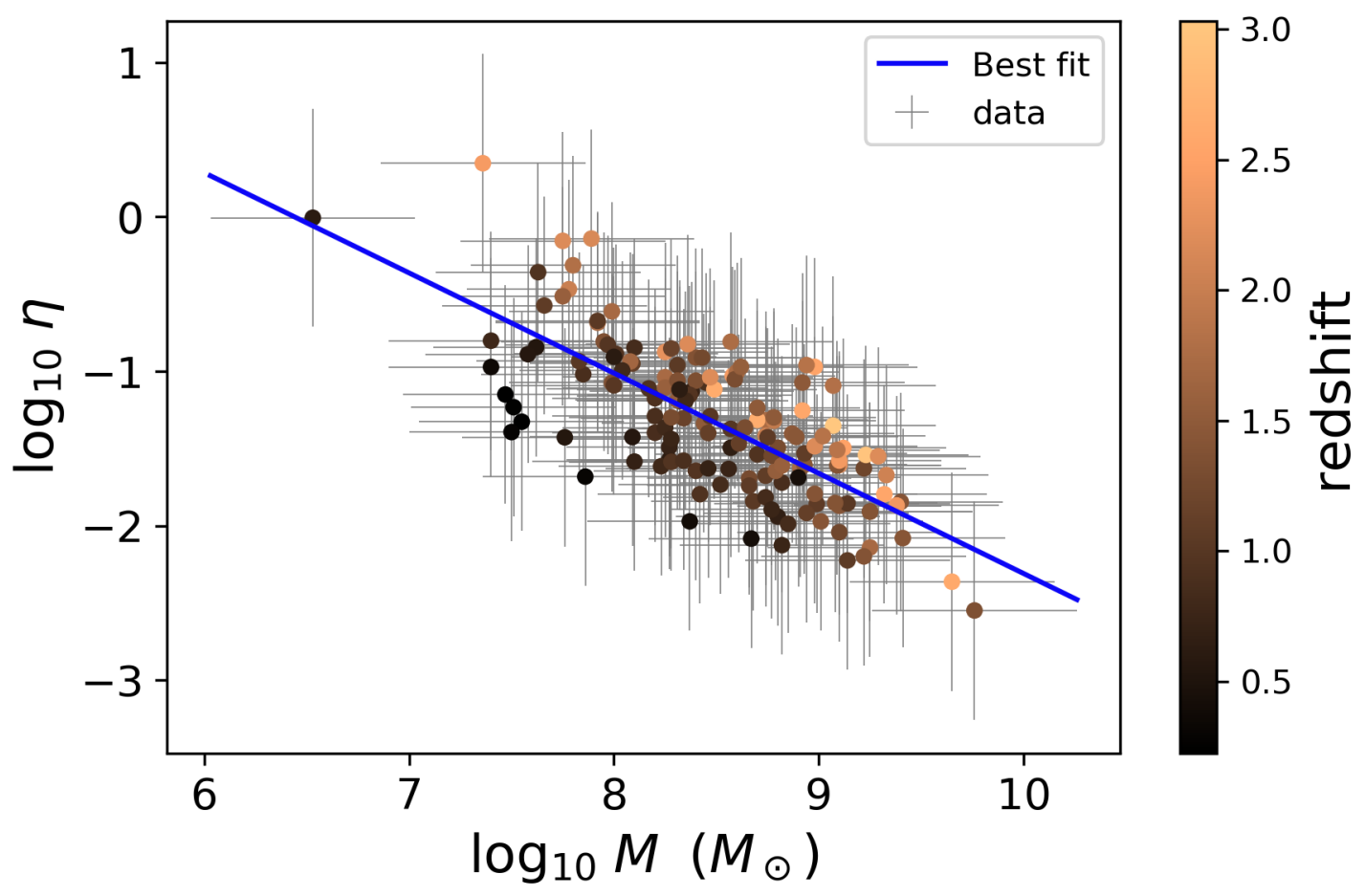

Figure 4.8: Jet production efficiencies as a function of BH mass. See caveats mentioned in Section 4.4.

In order to make more reliable assessments of the relation between $\eta$ and $M$, we need independent constraints on these variables, i.e. obtain $\eta$ independently from $M$.

\subsubsection{Black hole spins}

We searched the literature for the state-of-the-art models capable of explaining the launching of relativistic jets from thin accretion discs, as appropriate for jetted quasars. The most promising model for FSRQs corresponds to the GRMHD simulations of moderately thin MADs carried out by Avara, McKinney, and Reynolds (2016). Using data obtained from a variety of disc thicknesses, Avara, McKinney, and Reynolds (2016) obtained an empirical expression for the jet production efficiency,

$$
\eta_{\text {model }} \approx 4 \omega_{H}^{2}\left(1+\frac{0.3 \omega_{H}}{1+2 h^{4}}\right)^{2} h^{2}
$$


where $\omega_{H} \equiv a_{*} / r_{H}$ is the $\mathrm{BH}$ rotation frequency, $r_{H}=1+\sqrt{1-a_{*}^{2}}$ is the horizon radius, $h \equiv \arctan \left(c_{\mathrm{s}} / v_{\phi}\right) \approx H / R$ is the disc thickness, $c_{\mathrm{s}}$ is the sound speed and $v_{\phi}$ is the rotational speed (McKinney, Tchekhovskoy, and Blandford 2012; Avara, McKinney, and Reynolds 2016). The dependence $\eta \propto(H / R)^{2}$ was also reported in Penna et al. (2010). Thus we have a model that provides a full mapping of the observed jet efficiency to the spin and magnetic flux

$$
\eta=\eta_{\text {model }}\left(a_{*}, \phi\right)
$$

We proceed by solving this nonlinear equation, using the values of $\eta$ displayed in Figure 4.7 to constrain the $\mathrm{BH}$ spin-assuming of course that the Kerr metric is the correct description of the spacetime. When solving Equation (4.4.4), we follow Avara, McKinney, and Reynolds (2016) and adopt $h=0.13$.

We found that 36 objects- $24 \%$ of the sample-require $\eta$ larger than the maximum value allowed by Equation (4.4.4),

$$
\eta>\max \left(\eta_{\text {model }}\right)=\eta_{\text {model }}\left(a_{*}=0.998\right)=0.1
$$

where the maximum allowed spin is $\max \left(\mathrm{a}_{*}\right)=0.998$ (Thorne 1974). We discuss possible reasons for such large efficiencies in the next section.

We show in Figure 4.9 the lower limits on the jet efficiencies of the FSRQs. The horizontal line is the limiting efficiency assuming a black hole rotating with the maximum allowed spin, $a=0.998$, and also assuming a disc thickness $h=0.13$. Even considering the lower limits, there are still four objects with efficiencies which seem too high to be explained by the MAD model. We will consider possible explanations for this in the discussion.

For those objects which have $\eta<\max \left(\eta_{\text {model }}\right)$, we found an average spin of $\left\langle a_{*}\right\rangle=$ $a_{*}=0.84_{-0.25}^{+0.11}$. The distribution of spins is plotted in Figure 4.10, while Figure 4.11 plots the spins as a function of the the $\mathrm{BH}$ masses. We can see the tendency that the 


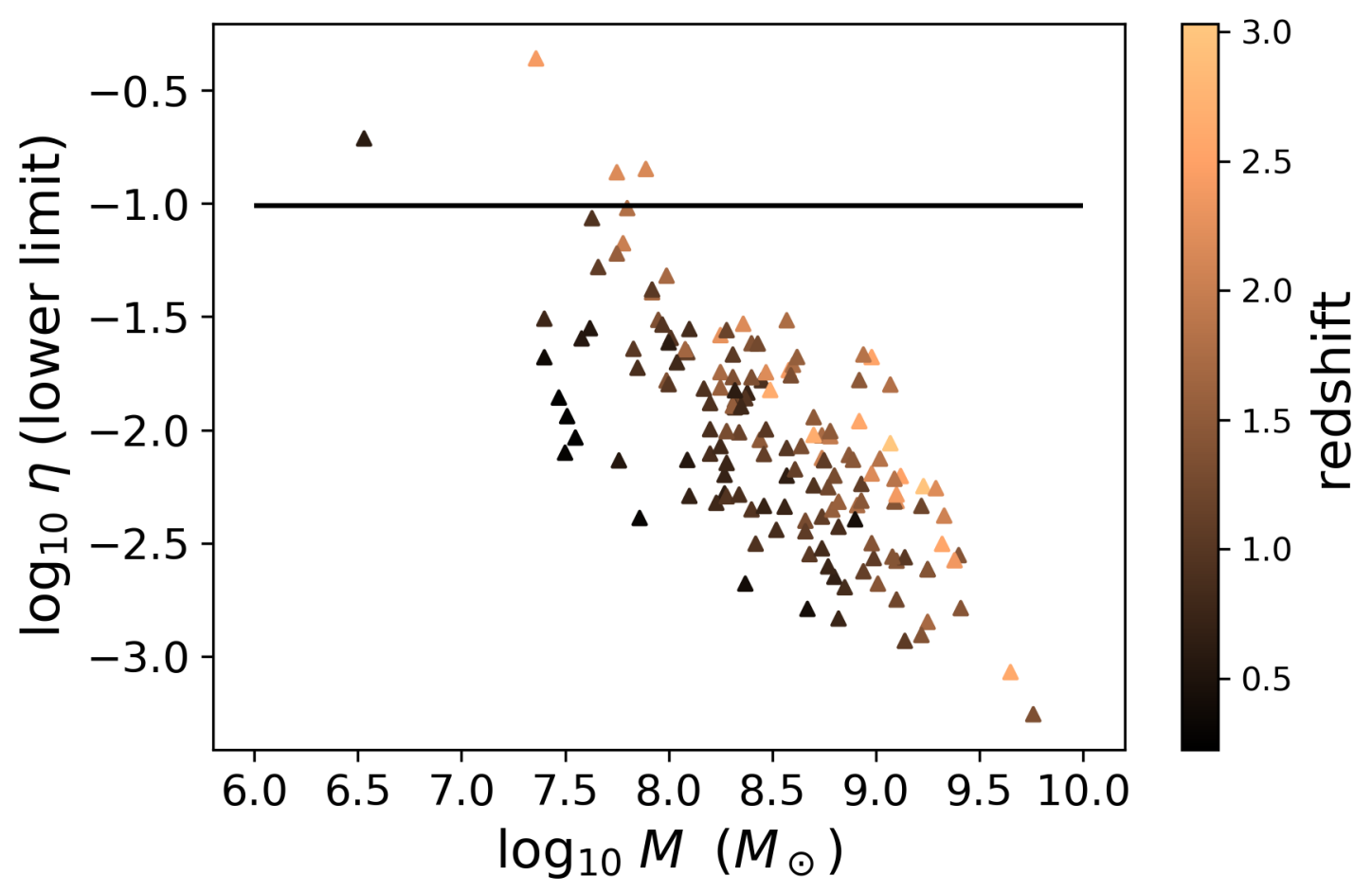

Figure 4.9: Lower limits on the jet efficiencies of the FSRQs. The horizontal line corresponds to the limiting efficiency assuming a maximum allowed spin and a disc thickness $h=0.13$ in Equation (4.4.4).

bigger the mass of the SMBH, the lower the associated spin. Because the values of $a_{*}$ are inferred from $\eta$, the same caveats involved in the $\eta-M$ correlation analysis also apply here: namely that a large degree of the anticorrelation between $a_{*}$ and $M$ occurs by construction.

The large uncertainties in $\eta_{\text {observed }}$ imply a somewhat large uncertainty of $\approx 0.2$ in the estimated spins. Thus, we decided to concentrate on the lower limits of our spins $a_{*}$, which average at 0.59 . Figure 4.12 displays the relation between the $\mathrm{BH}$ masses and the $1 \sigma$ lower limit on $a_{*}$. Figure 4.13 displays the distribution of lower limits on $a_{*}$.

We find that even considering the lower limits, 4 out of 154 objects (3\%) still have efficiencies which are too high for the model considered. These high-efficiency blazars are listed in Table 4.2 . 


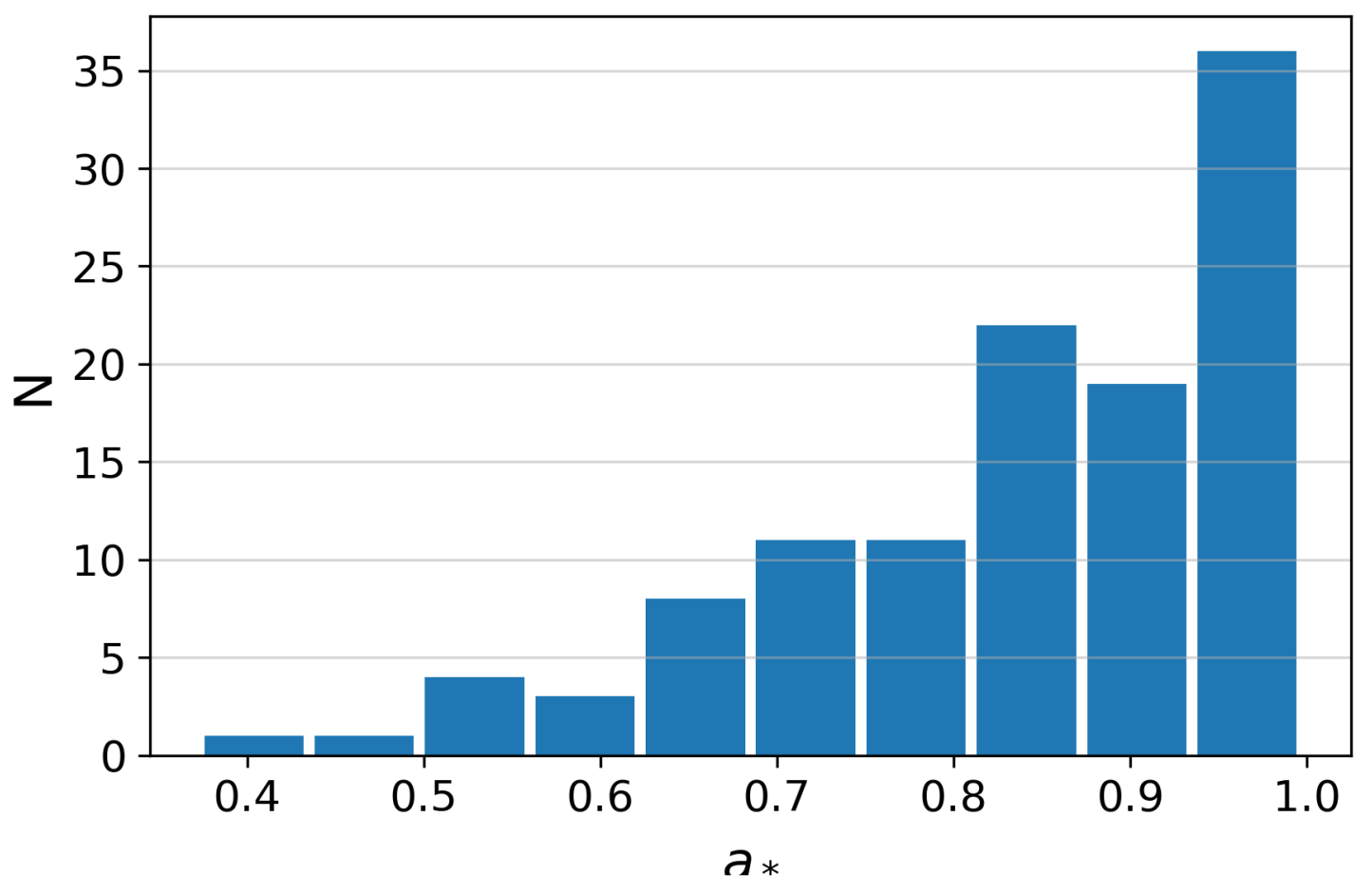

Figure 4.10: Distribution of spins.

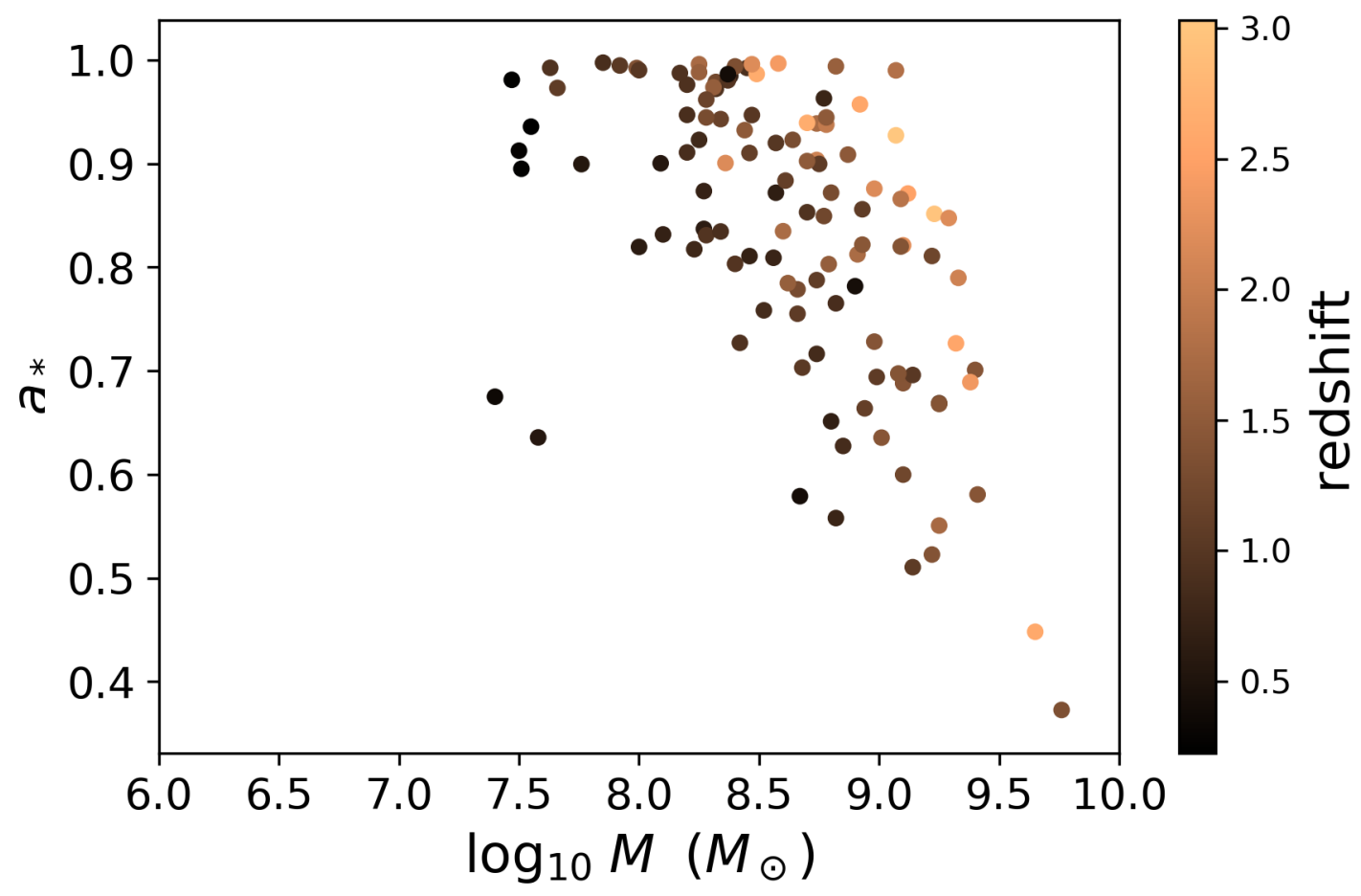

Figure 4.11: Spin estimates for the 118 FSRQs which have efficiencies consistent with the model we adopted. 


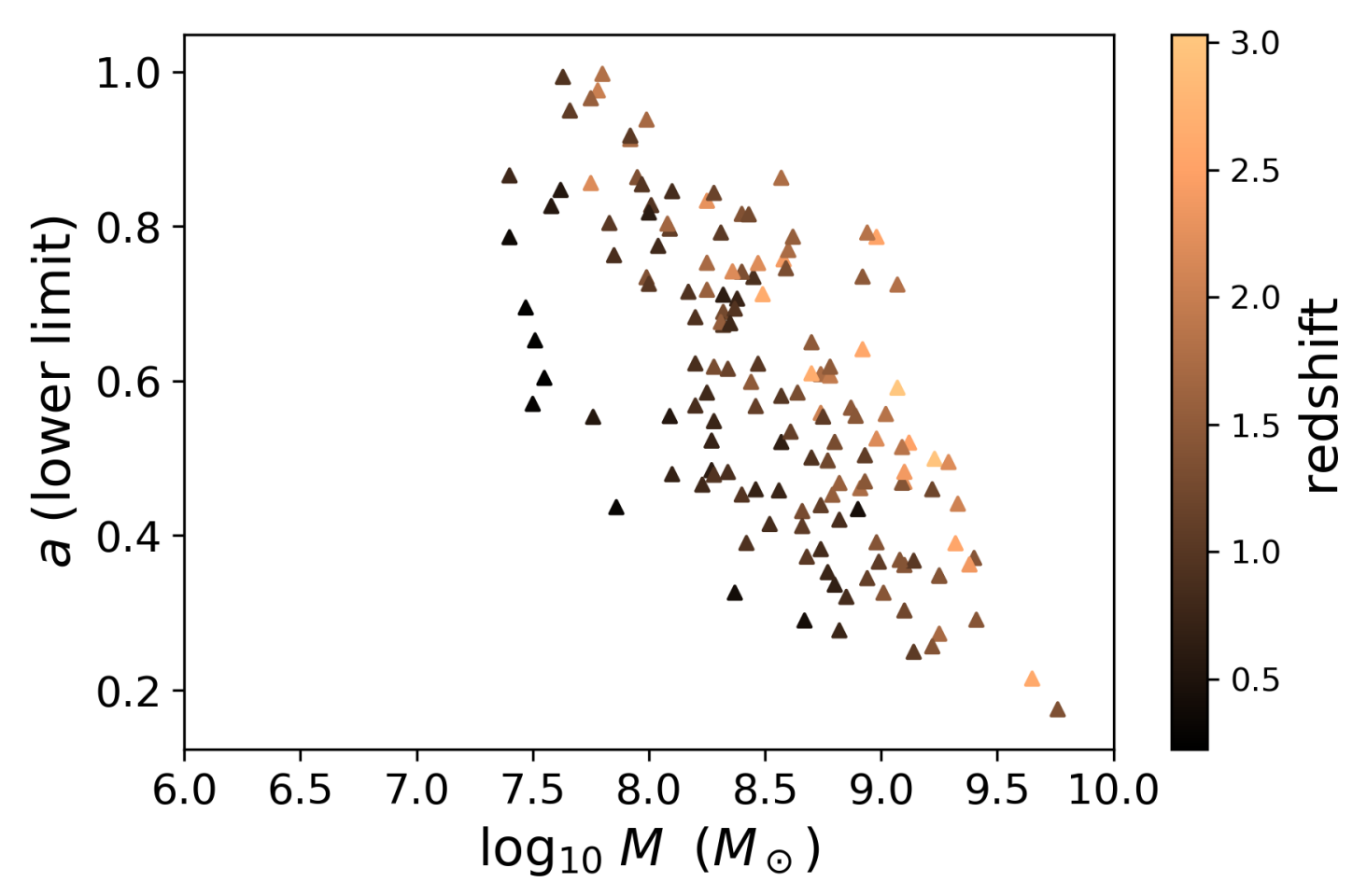

Figure 4.12: Spin lower limits as a function of the mass.

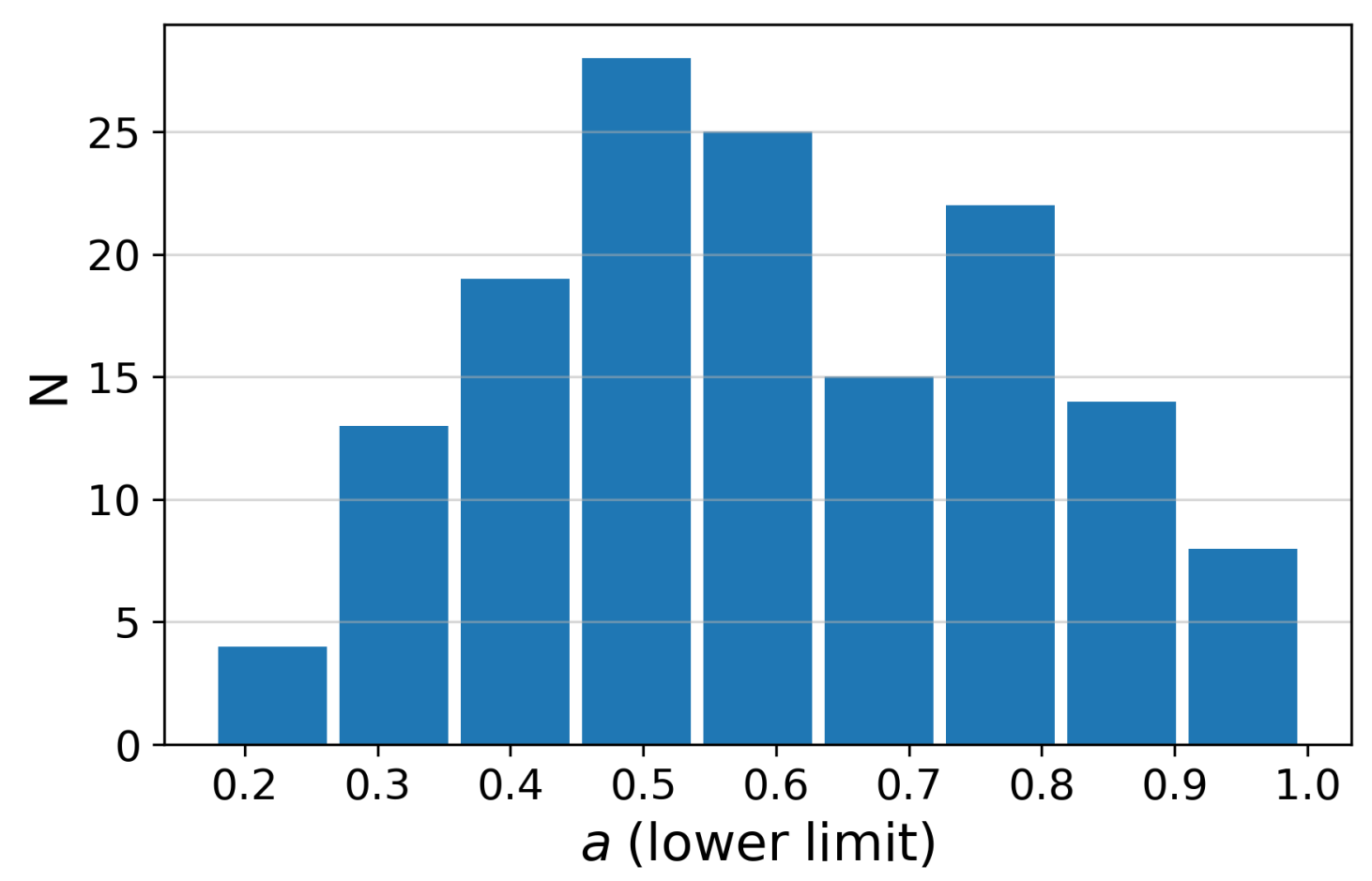

Figure 4.13: Distribution of spin lower limits. 
Table 4.2: The four high-efficiency blazars that cannot be explained by the simulationbased model considered.

\begin{tabular}{ccccc}
\hline $\begin{array}{c}\text { Object name } \\
\text { (4FGL) }\end{array}$ & $z$ & $\begin{array}{c}\log _{10} M \\
M_{\odot}\end{array}$ & $\eta$ & $\begin{array}{c}\eta \\
\text { (lower limit) }\end{array}$ \\
\hline 4FGL J0217.0-0821 & 0.607 & 6.53 & 0.986 & 0.194 \\
4FGL J0449.1+1121 & 2.153 & 7.89 & 0.723 & 0.142 \\
4FGL J0601.1-7035 & 2.409 & 7.36 & 2.225 & 0.437 \\
4FGL J2121.0+1901 & 2.18 & 7.75 & 0.699 & 0.137 \\
\hline
\end{tabular}

\subsection{Discussion}

There are several methods to estimate the jet power such as SED fitting (Ghisellini et al. 2014; Ghisellini and Tavecchio 2009), radio lobes emission assuming equipartition (Willott et al. 1999) and radio-core shift (Lobanov 1998; Shabala, Santoso, and Godfrey 2012). Pjanka, Zdziarski, and Sikora (2017) have shown that these different methods may yield a discrepancy of about one order of magnitude in the final estimates, although they could not conclude which method yields the most accurate result. Methods based on extended observations use average values taken over a larger timescale, whereas SED fitting methods use instantaneous - and often higher - values obtained in a short time frame (Pjanka, Zdziarski, and Sikora 2017).

Our method for deriving the the jet power is based on the $L_{\gamma}-P$ correlation of Nemmen et al. (2012) which is calibrated based on energetics of giant X-ray cavities inflated by jets over long period of time in radio galaxies (Cavagnolo et al. 2010; Meyer et al. 2011). Therefore, our method will systematically give lower values of $P$ reflecting the long-term average jet power, which are often lower than instantaneous estimates. For this reason, our P-estimates are lower than those of G14, who reported that the jet power is larger than the accretion power $P_{\text {acc }} \equiv \dot{M} c^{2}$ by a factor of $\sim 10$. This is clearly seen in Figure 4.14 which compares our results with the best-fit relation of G14.

In this work, our method for estimating the jet power relies on extended radio luminosities (Nemmen et al. 2012), and we found a mean jet efficiency of 0.096, with a relatively high uncertainty of 0.71 dex. It is interesting to note that, despite methods 


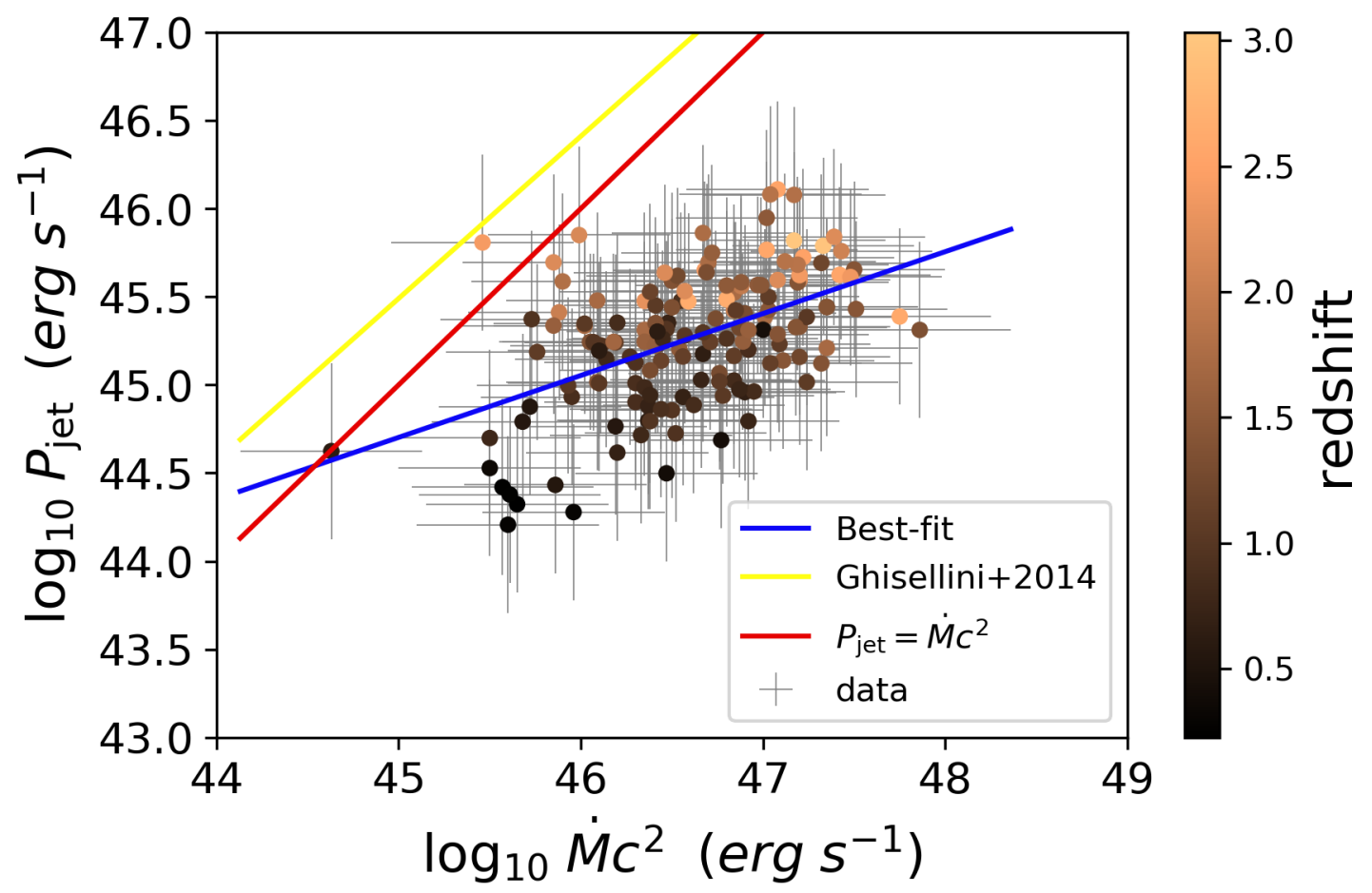

Figure 4.14: Jet powers versus the accretion power $\dot{M} c^{2}$. The Solid blue line indicates the best-fit relation of G14 and the dashed red line corresponds to $P=\dot{M} c^{2}$. The points are systematically below the G14 due to the method that we used to constrain $P$.

relying on extended radio luminosities having produced lower jet efficiencies, our results fall in the lower end of blazar studies that used SED fitting methods (i.e. G14), while being on average one order of magnitude higher than the values obtained by Inoue et al. (2017), who also used extended radio luminosities, as in Willott et al. (1999). We note, however, that within one sigma, our results for the jet efficiency are also compatible with those of van Velzen and Falcke (2013) and Inoue et al. (2017), although our findings of $a_{*}=0.84_{-0.25}^{+0.11}$ are higher than the spin distribution found in Inoue et al. (2017), who found $a_{*}=0.13_{-0.059}^{+0.11}$.

Pjanka, Zdziarski, and Sikora (2017) analyzed a sample of blazars - instead of a broader sample of objects classified simply as radio-loud quasars - and found that jet efficiencies obtained through SED fitting methods are about 10 times larger than those obtained via radio-lobe methods. The presence of $e^{ \pm}$pairs, for example, leads to a decrease in the jet power, and consequently in the jet efficiency. Moreover, radio-lobe 
methods rely on extended, long-term measurements, and the high variability of blazars over time could be playing a crucial role in leading to smaller values of jet efficiency.

We note that the adoption of larger estimates of $P_{\text {jet }}$ in this work, approaching those of G14, would result in a systematic increase in the values of $a_{*}$ in the sample - see Equation (4.4.4). Moreover, if the accretion rates are systematically lower than the value we assumed- $\dot{M}=0.01 \dot{M}_{\text {Edd }}$ instead of $\dot{M}=0.1 \dot{M}_{\text {Edd }}$-this would result in an increase in $\eta$, which would, in turn, also cause an increase in the spin values.

Indeed, if we adopt the jet powers as those estimated by G14, we find that the jet efficiencies of all objects are larger than the maximum efficiency allowed by the Avara, McKinney, and Reynolds (2016) model. If the jet powers of G14 reflect the true powers of blazars, then the jet model needs to be revised, otherwise the $\mathrm{BH}$ would not be able to power these jets. Either the accretion flow is systematically thicker and/or the accretion rates are systematically larger than we considered as discussed above (cf. also Section 4.5.2).

\subsubsection{Relation with SMBH and galaxy evolution}

Our results support larger values of spins, similar to what has been found in models of SMBH evolution and growth through mergers - e.g. Volonteri et al. (2005), Volonteri, Sikora, and Lasota (2007), and Volonteri et al. (2013) -, although not as many as 70\% of our black holes can be said to be maximally spinning, or close to it, as suggested by Volonteri et al. (2005). To investigate in more detail possible patterns in the spin evolution of the black holes in our sample, we divided them according to mass ranges and redshifts.

Figure 4.11 shows the spins distributed according to SMBH mass. We see a general tendency for the spins to decrease as the SMBH masses increase. Such a tendency has been suggested by King, Pringle, and Hofmann (2008), who argued that a series of accretion episodes, in which the discs are randomly oriented due to self-gravity, 
would spin down the SMBHs to around 0.1 to 0.3 , and also that mergers would have a short-lived influence on the spin values. However, even if our results point towards a decrease in spin as the mass increases, we note that we have obtained spins which are, on average, higher than those in King, Pringle, and Hofmann (2008). Moreover, while our results suggest moderate to high spins, we also found that some objects considered here display higher values of spins. These could result from mergers with black holes of similar mass and spin orientation. Such mergers are expected to have occurred at some point in the formation of giant elliptical galaxies.

This pattern of spin decreasing as the mass increases has also been found in cosmological simulations by Dubois, Volonteri, and Silk (2014), although they argue that the accretion disc's angular momentum is conserved, with spin decreasing due to accretion not being significant. Moreover, Dubois, Volonteri, and Silk (2014) also found that mass increases at lower redshifts would be due to more mergers, while accretion would be unable to spin up black holes at lower redshifts due to the gas in the accretion disc being quenched.

We note that Dubois, Volonteri, and Silk (2014) found that spins are high regardless of redshift. In particular, they found that for $z>2$ the spins are close to the maximum value, while at redshifts between 1 and 2, the spins decrease as the black holes undergo more mergers with other black holes of different spin orientations - and their masses increase - and accretion can no longer spin up the black holes. In our sample, we found no correlation between spins and redshifts, hence our results do not allow us to reach any substantial conclusions regarding a possible relation between these two quantities.

\subsubsection{The extremes of blazar jets}

Four out of 154 objects in the sample (3\%) display jets which are too powerful to be accounted by the MAD thin disc model of Avara, McKinney, and Reynolds (2016) with $h=0.13$ (cf. Table 4.2). Their efficiencies range between 0.7 and 2.2. 
One possibility to conciliate the model with the observations of these extreme blazars is if their accretion rates are higher than the value we assumed. For instance, if they have $\dot{M} \gtrsim 0.7 \dot{M}_{\mathrm{Edd}}$, then their efficiencies are lower than $\eta=0.1$ and can be explained by the jet model with $h=0.13$. Alternatively, we can also explain their efficiencies keeping $\dot{M}=0.1 \dot{M}_{\text {Edd }}$ and varying $h$ between 0.35 and 0.67 (or between 0.16 and 0.28 if we consider only the lower limits for $\eta$ ). These two possibilities might be related since as we increase $\dot{M}$, radiation pressure becomes more important and should make the disc thicker.

Finally, another possibility if these extreme blazars are accreting at $10 \%$ Eddington level and have $h \leq 0.1$ is that the Avara et al. model is inappropriate for them. This is discussed in the subsection that follows.

\subsubsection{Caveats}

One of the main results of this work is that GRMHD models of jet formation based on moderately thin accretion discs, in which the magnetic field threading the black hole horizon reaches the maximum value sustainable by the accreting matter, are able to explain the energetics of the majority of jetted beamed quasars.

Standard theory predicts a disc thickness $H / R \sim 0.01$ (Shakura and Sunyaev 1973; Abramowicz and Fragile 2013). Our assumption of $H / R=0.13$ is based on the availability of GRMHD simulations of moderately thin discs (Avara, McKinney, and Reynolds 2016). If FSRQs prefer very thin discs with $H / R<0.1$, then this would imply that Equation (4.4.4) does not describe adequately the energetics of jetted quasars. For instance, if we instead adopt $H / R=0.01$ we have $\max (\eta)=0.0006$ which would only be able to explain one single object in the sample, even considering the lower limits on $\eta_{\text {observed }}$

One way of reconciling the observations of powerful jetted quasars with the work of Avara, McKinney, and Reynolds (2016) is if the discs in quasars are indeed only 
moderately thin, perhaps due to slightly larger accretion rates than usually assumed, which would increase the radiation pressure effects and bloat up the discs similarly to supercritical discs. Higher-resolution simulations of very thin discs such as those of Liska et al. (2019) also including radiation pressure effects should help to clarify these issues.

Finally, we stress that one should be careful when interpreting the anticorrelations between (i) $\eta$ and $M$ (Figure 4.8), (ii) $a_{*}$ and $M$ (Figure 4.11) as physical trends. As described in Section 4.4, the anticorrelation $\eta \propto M^{-1}$ occurs by construction in our work due to our assumptions in estimating the jet efficiencies. Importantly, constraints of $\eta$ which do not depend on $M$ are needed.

\subsection{Conclusions}

In this work, we analyzed a sample of 154 beamed jetted quasars-flat spectrum radio quasars-with gamma-ray luminosities observed with Fermi-LAT and black hole masses estimated with a variety of methods. We used the beamed gamma luminosities as a proxy of their total jet powers and adopted standard quasar accretion rates of $10 \%$ Eddington, which allowed us to estimate their mass accretion rates from the $\mathrm{BH}$ masses. Our goal in this work was to test whether current models of jet formation from thin discs are able to explain the energetics in the sample and constrain the $\mathrm{BH}$ spin necessary to account for the jet powers. Our main conclusions can be summarized as follows:

(i) A median of $4 \%$ of the rest-mass energy associated with accreted matter is converted to jet power in jetted quasars, with the extremes being $0.3 \%$ and $200 \%$ on the low and high ends, respectively.

(ii) We find a correlation between $\mathrm{BH}$ mass and the observed $\gamma$-ray luminosity. This implies that $L_{\gamma}$ could potentially be used as a mass estimator in blazar $\gamma$-ray studies: 
$M=4 \times 10^{8}\left(L_{\gamma} / 10^{47} \mathrm{erg} \mathrm{s}^{-1}\right)^{0.37} M_{\odot}$. The resulting masses have an uncertainty of 0.5 dex.

(iii) Magnetically arrested, moderately thin $(H / R \sim 0.1)$ accretion discs around Kerr BHs are able to explain the energetics of the majority of jetted beamed quasars.

(iv) 3\% of the sample - four blazars - have efficiencies which are too high to be accounted by the model without significant changes to the parameters. Either their accretion rates are higher than assumed, the disc thickness is larger, or the jet model we adopted is inappropriate for them.

(v) By modeling our FSRQ sample with the MAD thin disc model, we obtained an average spin of 0.84 . The lowest spin on the sample within $1 \sigma$ is 0.2 .

(vi) Our spin estimates are compatible with results from models for the cosmological merger-driven evolution of SMBHs which support rapidly rotating black holes.

(vii) If accretion discs in quasars are considerable thinner than assumed here-i.e. if the discs have $H / R=0.01$ or lower-then MAD discs are unable to explain the energetics of the majority of the sample. If this is the case, then general relativistic models for jet production in quasars are missing an important ingredient and need considerable revision.

\subsection{Data}

Table 4.3 contains the data used in this work. 
Table 4.3: Data on the objects used in this work. Spins denoted by one asterisk $\left(^{*}\right)$ could not be obtained using the jet efficiency $\eta$ as input to the simulation-based model in Equation 4.4.4, while lower limit spins denoted by two asterisks ${ }^{* *}$ ) could not be obtained using the same equation for the lower limit jet efficiency $\eta_{\min }$ as input. The uncertainties in mass and jet power are 0.5 dex.

\begin{tabular}{|c|c|c|c|c|c|c|c|c|c|}
\hline $\begin{array}{l}\text { Object name } \\
\text { (4FGL) }\end{array}$ & $\begin{array}{c}\text { Object name } \\
\text { (G14/G15) }\end{array}$ & $z$ & $\begin{array}{c}\log _{10} M \\
M_{\odot}\end{array}$ & $\begin{array}{l}\log _{10} L_{\gamma} \\
\mathrm{erg} \mathrm{s}^{-1}\end{array}$ & $\begin{array}{c}\log _{10} P_{\text {jet }} \\
\text { erg s }^{-1}\end{array}$ & $\eta$ & $\begin{array}{c}\eta \\
\text { (lower limit) }\end{array}$ & $a_{*}$ & $\begin{array}{c}a_{*} \\
\text { (lower limit) }\end{array}$ \\
\hline 4FGL J0004.4-4737 & $0004-4736$ & 0.88 & 7.85 & 46.53 & 44.93 & 0.096 & 0.019 & 0.997 & 0.763 \\
\hline 4FGL J0011.4+0057 & $0011+0057$ & 1.493 & 8.44 & 47.07 & 45.2 & 0.046 & 0.009 & 0.932 & 0.599 \\
\hline 4FGL J0016.5+1702 & $0015+1700$ & 1.709 & 9.25 & 47.08 & 45.21 & 0.007 & 0.001 & 0.551 & 0.273 \\
\hline 4FGL J0017.5-0514 & 0017-0512 & 0.226 & 7.55 & 45.34 & 44.32 & 0.047 & 0.009 & 0.936 & 0.604 \\
\hline 4FGL J0023.7+4457 & $0023+4456$ & 2.023 & 7.78 & 47.47 & 45.41 & 0.34 & 0.067 & * & 0.976 \\
\hline 4FGL J0024.7+0349 & $0024+0349$ & 0.545 & 7.76 & 45.55 & 44.43 & 0.037 & 0.007 & 0.899 & 0.554 \\
\hline 4FGL J0042.2+2319 & $0042+2320$ & 1.426 & 9.01 & 46.94 & 45.14 & 0.011 & 0.002 & 0.636 & 0.326 \\
\hline 4FGL J0043.8+3425 & $0043+3426$ & 0.966 & 8.01 & 47.11 & 45.22 & 0.13 & 0.025 & * & 0.827 \\
\hline 4FGL J0044.2-8424 & $0044-8422$ & 1.032 & 8.68 & 46.55 & 44.94 & 0.014 & 0.003 & 0.703 & 0.373 \\
\hline 4FGL J0047.9+2233 & $0048+2235$ & 1.161 & 8.34 & 46.93 & 45.14 & 0.05 & 0.01 & 0.943 & 0.616 \\
\hline 4FGL J0050.4-0452 & 0050-0452 & 0.922 & 8.2 & 46.91 & 45.13 & 0.067 & 0.013 & 0.976 & 0.682 \\
\hline 4FGL J0058.4+3315 & $0058+3311$ & 1.369 & 7.99 & 46.7 & 45.02 & 0.084 & 0.017 & 0.992 & 0.734 \\
\hline 4FGL J0102.4+4214 & $0102+4214$ & 0.874 & 8.2 & 46.69 & 45.01 & 0.051 & 0.01 & 0.947 & 0.623 \\
\hline 4FGL J0102.8+5824 & $0102+5824$ & 0.644 & 8.57 & 47.01 & 45.18 & 0.032 & 0.006 & 0.872 & 0.521 \\
\hline 4FGL J0104.8-2416 & 0104-2416 & 1.747 & 8.91 & 47.42 & 45.39 & 0.024 & 0.005 & 0.812 & 0.461 \\
\hline 4FGL J0157.7-4614 & $0157-4614$ & 2.287 & 8.25 & 47.6 & 45.48 & 0.134 & 0.026 & * & 0.833 \\
\hline 4FGL J0217.0-0821 & 0217-0820 & 0.607 & 6.53 & 45.93 & 44.62 & 0.986 & 0.194 & * & $* *$ \\
\hline 4FGL J0226.5+0938 & $0226+0937$ & 2.605 & 9.65 & 47.43 & 45.39 & 0.004 & 0.001 & 0.448 & 0.215 \\
\hline 4FGL J0237.8+2848 & $0237+2848$ & 1.206 & 9.22 & 48.02 & 45.69 & 0.024 & 0.005 & 0.811 & 0.46 \\
\hline 4FGL J0245.4+2408 & $0245+2405$ & 2.243 & 9.1 & 47.83 & 45.59 & 0.025 & 0.005 & 0.821 & 0.469 \\
\hline 4FGL J0245.9-4650 & $0246-4651$ & 1.385 & 8.4 & 47.82 & 45.59 & 0.123 & 0.024 & * & 0.816 \\
\hline 4FGL J0252.8-2219 & 0252-2219 & 1.419 & 9.4 & 47.95 & 45.65 & 0.014 & 0.003 & 0.701 & 0.371 \\
\hline 4FGL J0253.9+5103 & $0253+5102$ & 1.732 & 8.74 & 47.69 & 45.52 & 0.048 & 0.009 & 0.939 & 0.609 \\
\hline 4FGL J0257.9-1215 & $0257-1212$ & 1.391 & 9.22 & 46.91 & 45.12 & 0.006 & 0.001 & 0.523 & 0.257 \\
\hline 4FGL J0303.6-6211 & 0303-6211 & 1.348 & 9.76 & 47.28 & 45.31 & 0.003 & 0.001 & 0.373 & 0.175 \\
\hline 4FGL J0309.9-6058 & 0309-6058 & 1.479 & 8.87 & 47.78 & 45.57 & 0.039 & 0.008 & 0.909 & 0.565 \\
\hline 4FGL J0315.9-1033 & 0315-1031 & 1.565 & 7.75 & 47.33 & 45.34 & 0.306 & 0.06 & * & 0.966 \\
\hline 4FGL J0325.7+2225 & $0325+2224$ & 2.066 & 9.33 & 48.15 & 45.76 & 0.021 & 0.004 & 0.79 & 0.441 \\
\hline 4FGL J0413.1-5332 & $0413-5332$ & 1.024 & 7.83 & 46.66 & 45.0 & 0.116 & 0.023 & $*$ & 0.804 \\
\hline 4FGL J0422.1-0644 & $0422-0643$ & 0.242 & 7.47 & 45.53 & 44.42 & 0.071 & 0.014 & 0.981 & 0.695 \\
\hline 4FGL J0438.4-1254 & $0438-1251$ & 1.285 & 8.66 & 46.8 & 45.07 & 0.02 & 0.004 & 0.779 & 0.432 \\
\hline 4FGL J0442.6-0017 & 0442-0017 & 0.845 & 8.1 & 47.36 & 45.35 & 0.142 & 0.028 & * & 0.846 \\
\hline 4FGL J0449.1+1121 & $0449+1121$ & 2.153 & 7.89 & 48.33 & 45.85 & 0.723 & 0.142 & * & $* *$ \\
\hline 4FGL J0456.6-3136 & 0456-3136 & 0.865 & 8.2 & 46.48 & 44.9 & 0.04 & 0.008 & 0.911 & 0.568 \\
\hline 4FGL J0507.7-6104 & 0507-6104 & 1.089 & 8.74 & 46.99 & 45.16 & 0.021 & 0.004 & 0.788 & 0.439 \\
\hline 4FGL J0509.4+1012 & $0509+1011$ & 0.621 & 8.27 & 46.27 & 44.8 & 0.027 & 0.005 & 0.837 & 0.485 \\
\hline 4FGL J0526.2-4830 & $0526-4830$ & 1.3 & 8.8 & 47.46 & 45.41 & 0.032 & 0.006 & 0.872 & 0.521 \\
\hline 4FGL J0532.6+0732 & $0532+0732$ & 1.254 & 8.43 & 47.88 & 45.62 & 0.123 & 0.024 & * & 0.815 \\
\hline 4FGL J0532.9-8325 & $0533-8324$ & 0.784 & 7.4 & 46.08 & 44.7 & 0.158 & 0.031 & * & 0.866 \\
\hline 4FGL J0533.3+4823 & $0533+4822$ & 1.16 & 9.25 & 47.53 & 45.44 & 0.012 & 0.002 & 0.669 & 0.348 \\
\hline 4FGL J0541.6-0541 & 0541-0541 & 0.838 & 8.74 & 46.71 & 45.02 & 0.015 & 0.003 & 0.716 & 0.382 \\
\hline 4FGL J0601.1-7035 & $0601-7036$ & 2.409 & 7.36 & 48.25 & 45.81 & 2.225 & 0.437 & * & $* *$ \\
\hline 4FGL J0608.0-0835 & 0607-0834 & 0.87 & 8.82 & 47.06 & 45.2 & 0.019 & 0.004 & 0.765 & 0.42 \\
\hline 4FGL J0608.1-1521 & $0608-1520$ & 1.094 & 8.09 & 47.14 & 45.24 & 0.112 & 0.022 & $*$ & 0.797 \\
\hline 4FGL J0625.8-5441 & $0625-5438$ & 2.051 & 8.74 & 47.5 & 45.42 & 0.038 & 0.008 & 0.904 & 0.559 \\
\hline 4FGL J0654.4+4514 & $0654+4514$ & 0.928 & 8.17 & 46.98 & 45.16 & 0.077 & 0.015 & 0.987 & 0.715 \\
\hline 4FGL J0654.3+5042 & $0654+5042$ & 1.253 & 8.32 & 47.17 & 45.26 & 0.069 & 0.014 & 0.979 & 0.689 \\
\hline 4FGL J0713.8+1935 & $0713+1935$ & 0.54 & 7.62 & 46.42 & 44.88 & 0.143 & 0.028 & * & 0.847 \\
\hline 4FGL J0721.3+0405 & $0721+0406$ & 0.665 & 8.8 & 46.59 & 44.96 & 0.011 & 0.002 & 0.651 & 0.336 \\
\hline 4FGL J0723.5+2900 & $0723+2859$ & 0.966 & 8.4 & 46.38 & 44.86 & 0.023 & 0.004 & 0.803 & 0.453 \\
\hline 4FGL J0725.2+1425 & $0725+1425$ & 1.038 & 8.31 & 47.55 & 45.45 & 0.11 & 0.022 & * & 0.792 \\
\hline 4FGL J0746.4+2546 & $0746+2549$ & 2.979 & 9.23 & 48.21 & 45.79 & 0.029 & 0.006 & 0.852 & 0.499 \\
\hline 4FGL J0805.4+6147 & $0805+6144$ & 3.033 & 9.07 & 48.27 & 45.82 & 0.044 & 0.009 & 0.927 & 0.591 \\
\hline 4FGL J0824.7+5552 & $0825+5555$ & 1.418 & 9.1 & 47.31 & 45.33 & 0.013 & 0.003 & 0.688 & 0.362 \\
\hline 4FGL J0830.8+2410 & $0830+2410$ & 0.942 & 8.7 & 47.18 & 45.26 & 0.029 & 0.006 & 0.853 & 0.501 \\
\hline 4FGL J0909.1+0121 & $0909+0121$ & 1.026 & 9.14 & 47.42 & 45.38 & 0.014 & 0.003 & 0.696 & 0.367 \\
\hline 4FGL J0910.6+2247 & $0910+2248$ & 2.661 & 8.7 & 47.62 & 45.49 & 0.048 & 0.01 & 0.939 & 0.61 \\
\hline 4FGL J0912.2+4127 & $0912+4126$ & 2.563 & 9.32 & 47.89 & 45.62 & 0.016 & 0.003 & 0.727 & 0.39 \\
\hline 4FGL J0920.9+4441 & $0920+4441$ & 2.189 & 9.29 & 48.31 & 45.84 & 0.028 & 0.006 & 0.847 & 0.495 \\
\hline 4FGL J0921.6+6216 & $0921+6215$ & 1.453 & 8.93 & 47.5 & 45.42 & 0.025 & 0.005 & 0.822 & 0.47 \\
\hline
\end{tabular}




\begin{tabular}{|c|c|c|c|c|c|c|c|c|c|}
\hline $\begin{array}{l}\text { Object name } \\
\text { (4FGL) }\end{array}$ & $\begin{array}{c}\text { Object name } \\
(\mathrm{G} 14 / \mathrm{G} 15)\end{array}$ & $z$ & $\begin{array}{c}\log _{10} M \\
M_{\odot}\end{array}$ & $\begin{array}{l}\log _{10} L_{\gamma} \\
\mathrm{erg} \mathrm{s}^{-1}\end{array}$ & $\begin{array}{c}\log _{10} P_{\text {jet }} \\
\text { erg s }^{-1}\end{array}$ & $\eta$ & $\begin{array}{c}\eta \\
\text { (lower limit) }\end{array}$ & $a_{*}$ & $\begin{array}{c}a_{*} \\
\text { (lower limit) }\end{array}$ \\
\hline 4FGL J0924.0+2816 & $0923+2815$ & 0.744 & 8.82 & 46.26 & 44.79 & 0.007 & 0.001 & 0.558 & 0.277 \\
\hline 4FGL J0923.5+4125 & $0923+4125$ & 1.732 & 7.92 & 47.32 & 45.34 & 0.206 & 0.041 & * & 0.913 \\
\hline 4FGL J0937.1+5008 & $0937+5008$ & 0.276 & 7.5 & 45.11 & 44.21 & 0.04 & 0.008 & 0.912 & 0.57 \\
\hline 4FGL J0946.6+1016 & 0946+1017 & 1.006 & 8.47 & 47.22 & 45.28 & 0.051 & 0.01 & 0.947 & 0.622 \\
\hline 4FGL J0949.2+1749 & $0949+1752$ & 0.693 & 8.1 & 45.91 & 44.62 & 0.026 & 0.005 & 0.832 & 0.479 \\
\hline 4FGL J0956.7+2516 & $0956+2515$ & 0.708 & 8.46 & 46.53 & 44.93 & 0.024 & 0.005 & 0.811 & 0.46 \\
\hline 4FGL J0957.6+5523 & $0957+5522$ & 0.899 & 8.45 & 47.6 & 45.48 & 0.084 & 0.017 & 0.992 & 0.735 \\
\hline 4FGL J1012.7+2439 & $1012+2439$ & 1.8 & 7.8 & 47.82 & 45.59 & 0.486 & 0.095 & * & 0.997 \\
\hline 4FGL J1016.0+0512 & $1016+0513$ & 1.714 & 7.99 & 47.6 & 45.48 & 0.244 & 0.048 & * & 0.938 \\
\hline 4FGL J1018.4+3540 & $1018+3542$ & 1.228 & 9.1 & 46.98 & 45.16 & 0.009 & 0.002 & 0.6 & 0.303 \\
\hline 4FGL J1033.9+6050 & $1032+6051$ & 1.064 & 8.75 & 47.5 & 45.42 & 0.037 & 0.007 & 0.9 & 0.554 \\
\hline 4FGL J1033.1+4115 & $1033+4116$ & 1.117 & 8.61 & 47.14 & 45.24 & 0.034 & 0.007 & 0.884 & 0.534 \\
\hline 4FGL J1033.9+6050 & $1033+6051$ & 1.401 & 9.09 & 47.81 & 45.58 & 0.025 & 0.005 & 0.82 & 0.468 \\
\hline 4FGL J1037.7-2822 & $1037-2823$ & 1.066 & 8.99 & 47.12 & 45.23 & 0.014 & 0.003 & 0.694 & 0.366 \\
\hline 4FGL J1043.2+2408 & $1043+2408$ & 0.559 & 8.09 & 46.21 & 44.76 & 0.038 & 0.007 & 0.9 & 0.555 \\
\hline 4FGL J1106.0+2813 & $1106+2812$ & 0.843 & 8.85 & 46.59 & 44.96 & 0.01 & 0.002 & 0.627 & 0.321 \\
\hline 4FGL J1112.5+3448 & $1112+3446$ & 1.956 & 8.78 & 47.76 & 45.56 & 0.048 & 0.009 & 0.938 & 0.607 \\
\hline 4FGL J1124.0+2336 & $1124+2336$ & 1.549 & 8.79 & 47.15 & 45.25 & 0.023 & 0.004 & 0.803 & 0.453 \\
\hline 4FGL J1146.9+3958 & $1146+3958$ & 1.088 & 8.93 & 47.64 & 45.5 & 0.029 & 0.006 & 0.856 & 0.504 \\
\hline 4FGL J1152.3-0839 & $1152-0841$ & 2.367 & 9.38 & 47.87 & 45.61 & 0.014 & 0.003 & 0.689 & 0.363 \\
\hline 4FGL J1154.0+6018 & $1154+6022$ & 1.12 & 8.94 & 46.91 & 45.12 & 0.012 & 0.002 & 0.664 & 0.345 \\
\hline 4FGL J1159.5+2914 & $1159+2914$ & 0.725 & 8.38 & 47.36 & 45.35 & 0.074 & 0.015 & 0.985 & 0.706 \\
\hline 4FGL J1208.9+5441 & $1208+5441$ & 1.344 & 8.4 & 47.53 & 45.44 & 0.087 & 0.017 & 0.994 & 0.741 \\
\hline 4FGL J1209.8+1810 & $1209+1810$ & 0.845 & 8.52 & 46.44 & 44.89 & 0.018 & 0.004 & 0.758 & 0.415 \\
\hline 4FGL J1222.5+0414 & $1222+0413$ & 0.966 & 8.37 & 47.29 & 45.32 & 0.07 & 0.014 & 0.98 & 0.693 \\
\hline 4FGL J1224.9+2122 & $1224+2122$ & 0.434 & 8.9 & 47.28 & 45.31 & 0.021 & 0.004 & 0.782 & 0.434 \\
\hline 4FGL J1223.9+5000 & $1224+5001$ & 1.065 & 8.66 & 46.71 & 45.02 & 0.018 & 0.004 & 0.755 & 0.412 \\
\hline 4FGL J1228.7+4858 & $1228+4858$ & 1.722 & 8.25 & 47.28 & 45.31 & 0.092 & 0.018 & 0.996 & 0.753 \\
\hline 4FGL J1239.5+0443 & $1239+0443$ & 1.761 & 8.57 & 48.36 & 45.86 & 0.155 & 0.03 & * & 0.863 \\
\hline 4FGL J1257.8+3228 & $1257+3229$ & 0.806 & 8.25 & 46.64 & 44.99 & 0.043 & 0.008 & 0.923 & 0.585 \\
\hline 4FGL J1303.6-4622 & $1303-4621$ & 1.664 & 8.08 & 47.14 & 45.24 & 0.116 & 0.023 & * & 0.803 \\
\hline 4FGL J1310.5+3221 & $1310+3220$ & 0.997 & 8.57 & 47.25 & 45.3 & 0.042 & 0.008 & 0.92 & 0.581 \\
\hline 4FGL J1317.6+3428 & $1317+3425$ & 1.055 & 9.14 & 46.7 & 45.02 & 0.006 & 0.001 & 0.51 & 0.25 \\
\hline 4FGL J1321.1+2216 & $1321+2216$ & 0.943 & 8.32 & 47.11 & 45.23 & 0.064 & 0.013 & 0.972 & 0.673 \\
\hline 4FGL J1326.9+2210 & $1327+2210$ & 1.403 & 9.25 & 47.53 & 45.44 & 0.012 & 0.002 & 0.668 & 0.348 \\
\hline 4FGL J1332.6-1256 & $1332-1256$ & 1.492 & 8.78 & 47.81 & 45.58 & 0.05 & 0.01 & 0.945 & 0.618 \\
\hline 4FGL J1333.7+5056 & $1333+5057$ & 1.362 & 7.95 & 47.14 & 45.24 & 0.156 & 0.031 & * & 0.864 \\
\hline 4FGL J1343.6+5755 & $1343+5754$ & 0.933 & 8.42 & 46.13 & 44.72 & 0.016 & 0.003 & 0.727 & 0.39 \\
\hline 4FGL J1344.2-1723 & $1344-1723$ & 2.506 & 9.12 & 48.09 & 45.72 & 0.032 & 0.006 & 0.871 & 0.52 \\
\hline 4FGL J1345.5+4453 & $1345+4452$ & 2.534 & 8.98 & 48.84 & 46.11 & 0.107 & 0.021 & * & 0.786 \\
\hline 4FGL J1347.6-3751 & $1347-3750$ & 1.3 & 8.28 & 46.83 & 45.08 & 0.05 & 0.01 & 0.945 & 0.618 \\
\hline 4FGL J1350.8+3033 & $1350+3034$ & 0.712 & 8.27 & 46.43 & 44.88 & 0.032 & 0.006 & 0.873 & 0.523 \\
\hline 4FGL J1358.1+7642 & $1357+7643$ & 1.585 & 8.25 & 47.15 & 45.24 & 0.078 & 0.015 & 0.988 & 0.718 \\
\hline 4FGL J1359.1+5544 & $1359+5544$ & 1.014 & 8.0 & 46.68 & 45.01 & 0.081 & 0.016 & 0.99 & 0.726 \\
\hline 4FGL J1423.5-7829 & $1423-7829$ & 0.788 & 8.23 & 46.11 & 44.72 & 0.024 & 0.005 & 0.817 & 0.466 \\
\hline 4FGL J1436.9+2321 & $1436+2321$ & 1.548 & 8.31 & 47.11 & 45.22 & 0.065 & 0.013 & 0.974 & 0.676 \\
\hline 4FGL J1438.9+3710 & $1438+3710$ & 2.399 & 8.58 & 47.94 & 45.65 & 0.094 & 0.018 & 0.997 & 0.757 \\
\hline 4FGL J1441.6-1522 & $1441-1523$ & 2.642 & 8.49 & 47.6 & 45.47 & 0.076 & 0.015 & 0.986 & 0.712 \\
\hline 4FGL J1443.9+2501 & $1443+2501$ & 0.939 & 7.63 & 47.4 & 45.37 & 0.439 & 0.086 & * & 0.993 \\
\hline 4FGL J1504.4+1029 & $1504+1029$ & 1.839 & 8.94 & 48.79 & 46.08 & 0.11 & 0.022 & * & 0.792 \\
\hline 4FGL J1522.1+3144 & $1522+3144$ & 1.484 & 8.92 & 48.52 & 45.95 & 0.085 & 0.017 & 0.992 & 0.735 \\
\hline 4FGL J1539.6+2743 & $1539+2744$ & 2.191 & 8.47 & 47.71 & 45.53 & 0.092 & 0.018 & 0.996 & 0.753 \\
\hline 4FGL J1549.5+0236 & $1549+0237$ & 0.414 & 8.67 & 46.05 & 44.69 & 0.008 & 0.002 & 0.579 & 0.29 \\
\hline 4FGL J1550.7+0528 & $1550+0527$ & 1.417 & 8.98 & 47.23 & 45.29 & 0.016 & 0.003 & 0.728 & 0.391 \\
\hline 4FGL J1553.6+1257 & $1553+1256$ & 1.308 & 8.64 & 47.4 & 45.38 & 0.043 & 0.008 & 0.923 & 0.585 \\
\hline 4FGL J1608.7+1029 & $1608+1029$ & 1.232 & 8.77 & 47.3 & 45.32 & 0.028 & 0.006 & 0.849 & 0.497 \\
\hline 4FGL J1613.6+3411 & $1613+3412$ & 1.4 & 9.08 & 47.31 & 45.33 & 0.014 & 0.003 & 0.697 & 0.369 \\
\hline 4FGL J1616.6+4630 & $1616+4632$ & 0.95 & 8.28 & 46.26 & 44.79 & 0.026 & 0.005 & 0.831 & 0.479 \\
\hline 4FGL J1617.3-5849 & $1617-5848$ & 1.422 & 9.41 & 47.51 & 45.43 & 0.008 & 0.002 & 0.581 & 0.291 \\
\hline 4FGL J1628.8-6149 & $1628-6152$ & 2.578 & 8.92 & 48.17 & 45.77 & 0.056 & 0.011 & 0.957 & 0.641 \\
\hline 4FGL J1635.2+3808 & $1635+3808$ & 1.813 & 9.07 & 48.78 & 46.08 & 0.081 & 0.016 & 0.99 & 0.725 \\
\hline 4FGL J1637.7+4717 & $1637+4717$ & 0.735 & 8.56 & 46.72 & 45.03 & 0.023 & 0.005 & 0.809 & 0.458 \\
\hline
\end{tabular}




\begin{tabular}{|c|c|c|c|c|c|c|c|c|c|}
\hline $\begin{array}{c}\text { Object name } \\
\text { (4FGL) }\end{array}$ & $\begin{array}{c}\text { Object name } \\
\text { (G14/G15) }\end{array}$ & $z$ & $\begin{array}{c}\log _{10} M \\
M_{\odot}\end{array}$ & $\begin{array}{l}\log _{10} L_{\gamma} \\
\operatorname{erg~s}^{-1}\end{array}$ & $\begin{array}{c}\log _{10} P_{\text {jet }} \\
\text { erg s }^{-1}\end{array}$ & $\eta$ & $\begin{array}{c}\eta \\
\text { (lower limit) }\end{array}$ & $a_{*}$ & $\begin{array}{c}a_{*} \\
\text { (lower limit) }\end{array}$ \\
\hline 4FGL J1703.6-6213 & $1703-6212$ & 1.747 & 8.6 & 48.03 & 45.7 & 0.099 & 0.019 & 0.998 & 0.769 \\
\hline 4FGL J1709.7+4318 & $1709+4318$ & 1.027 & 7.92 & 47.35 & 45.35 & 0.212 & 0.042 & . & 0.917 \\
\hline 4FGL J1734.3+3858 & $1734+3857$ & 0.975 & 7.97 & 47.14 & 45.24 & 0.149 & 0.029 & * & 0.854 \\
\hline 4FGL J1736.6+0628 & $1736+0631$ & 2.387 & 9.1 & 47.89 & 45.62 & 0.026 & 0.005 & 0.835 & 0.482 \\
\hline 4FGL J1802.6-3940 & $1802-3940$ & 1.319 & 8.59 & 47.92 & 45.64 & 0.089 & 0.017 & 0.995 & 0.746 \\
\hline 4FGL J1818.6+0903 & $1818+0903$ & 0.354 & 7.4 & 45.74 & 44.53 & 0.107 & 0.021 & * & 0.786 \\
\hline 4FGL J1830.1+0617 & $1830+0619$ & 0.745 & 8.77 & 46.62 & 44.97 & 0.013 & 0.002 & 0.675 & 0.353 \\
\hline 4FGL J1848.4+3217 & $1848+3219$ & 0.8 & 8.04 & 46.95 & 45.15 & 0.101 & 0.02 & 0.999 & 0.775 \\
\hline 4FGL J1902.9-6748 & $1903-6749$ & 0.254 & 7.51 & 45.45 & 44.38 & 0.059 & 0.012 & 0.963 & 0.652 \\
\hline 4FGL J1955.2+1358 & $1955+1358$ & 0.743 & 8.28 & 46.55 & 44.94 & 0.036 & 0.007 & 0.895 & 0.548 \\
\hline 4FGL J1959.1-4247 & $1959-4246$ & 2.178 & 8.98 & 47.83 & 45.59 & 0.033 & 0.006 & 0.876 & 0.525 \\
\hline 4FGL J2026.0-2845 & $2025-2845$ & 0.884 & 8.34 & 46.4 & 44.86 & 0.026 & 0.005 & 0.834 & 0.482 \\
\hline 4FGL J2035.4+1056 & $2035+1056$ & 0.601 & 8.0 & 47.05 & 45.19 & 0.124 & 0.024 & * & 0.818 \\
\hline 4FGL J2110.3+0808 & $2110+0809$ & 1.58 & 8.82 & 47.27 & 45.31 & 0.025 & 0.005 & 0.82 & 0.468 \\
\hline 4FGL J2121.0+1901 & $2121+1901$ & 2.18 & 7.75 & 48.03 & 45.7 & 0.699 & 0.137 & * & $* *$ \\
\hline 4FGL J2135.3-5006 & $2135-5006$ & 2.181 & 8.36 & 47.91 & 45.64 & 0.15 & 0.029 & * & 0.856 \\
\hline 4FGL J2145.0-3356 & $2145-3357$ & 1.361 & 8.31 & 47.35 & 45.35 & 0.087 & 0.017 & 0.994 & 0.742 \\
\hline 4FGL J2157.5+3127 & $2157+3127$ & 1.448 & 8.89 & 47.78 & 45.57 & 0.038 & 0.007 & 0.901 & 0.555 \\
\hline 4FGL J2201.5-8339 & $2202-8338$ & 1.865 & 9.09 & 48.0 & 45.68 & 0.031 & 0.006 & 0.866 & 0.514 \\
\hline 4FGL J2212.0+2356 & $2212+2355$ & 1.125 & 8.46 & 46.98 & 45.16 & 0.04 & 0.008 & 0.91 & 0.567 \\
\hline 4FGL J2219.2+1806 & $2219+1806$ & 1.071 & 7.66 & 47.03 & 45.19 & 0.267 & 0.052 & * & 0.95 \\
\hline 4FGL J2229.7-0832 & 2229-0832 & 1.56 & 8.62 & 48.14 & 45.75 & 0.107 & 0.021 & * & 0.787 \\
\hline 4FGL J2236.3+2828 & $2236+2828$ & 0.79 & 8.35 & 47.18 & 45.26 & 0.065 & 0.013 & 0.973 & 0.675 \\
\hline 4FGL J2237.0-3921 & $2237-3921$ & 0.297 & 7.86 & 45.25 & 44.28 & 0.021 & 0.004 & 0.785 & 0.437 \\
\hline 4FGL J2244.2+4057 & $2244+4057$ & 1.171 & 8.28 & 47.7 & 45.53 & 0.141 & 0.028 & * & 0.843 \\
\hline 4FGL J2321.9+3204 & $2321+3204$ & 1.489 & 8.7 & 47.77 & 45.56 & 0.058 & 0.011 & 0.962 & 0.65 \\
\hline 4FGL J2327.5+0939 & $2327+0940$ & 1.841 & 9.02 & 48.04 & 45.7 & 0.038 & 0.007 & 0.902 & 0.557 \\
\hline 4FGL J2331.0-2147 & $2331-2148$ & 0.563 & 7.58 & 46.26 & 44.79 & 0.129 & 0.025 & * & 0.826 \\
\hline 4FGL J2334.2+0736 & $2334+0736$ & 0.401 & 8.37 & 45.68 & 44.5 & 0.011 & 0.002 & 0.636 & 0.326 \\
\hline 4FGL J2345.2-1555 & 2345-1555 & 0.621 & 8.32 & 47.26 & 45.3 & 0.076 & 0.015 & 0.986 & 0.712 \\
\hline
\end{tabular}


84CHAPTER 4. JET EFFICIENCIES AND BLACK HOLE SPINS IN JETTED QUASARS 


\section{Chapter 5}

\section{Current Sheets in Jets}

A paper based on this chapter is in preparation and will be submitted soon.

\section{Summary of this Chapter}

Non-thermal emission is instrumental to understand blazar emission as well as flares coming from Sgr $A^{*}$. Motivated by a series of recent particle-in-cell (PIC) studies of magnetic reconnection as a source of non-thermal emission, we carried out general relativistic magnetohydrodynamical (GRMHD) simulations of hot accretion flows with different magnetic field topologies. We performed four simulations, exploring two different magnetic field topologies magnetically arrested disc (MAD) and a stable and normal evolution (SANE) - as well as slow $\left(a_{*}=0.1\right)$ and fast $\left(a_{*}=0.9\right)$ rotating black holes. All the simulations show the appearance of current sheets in the accretion disc, while some simuations show current sheets in the jet. 


\subsection{Introduction}

The presence of jets is an underlying feature of radio-loud AGN, such as M87 and Centaurus A, and including blazars. The very high speeds with which particles are launched in these jets make them naturally relativistic environments. The relativistic nature of these jets accounts for a series of effects which are typical of blazars, such as their very high brightness and an apparent superluminal motion.

Observations also suggest that the radiating particles are accelerated into a powerlaw distribution. The typical blazar SED contains two humps, a low-energy component and a high-energy one. The low-energy hump results from synchrotron radiation emitted by relativistic electrons and may extend from radio up to the UV. A power-law is required to explain the shape of this hump, which suggests the presence of electrons. The high-energy hump extends from X-rays to gamma-rays and is attributed to inverse Compton emission.

Theoretical studies of relativistic jets often employ GRMHD and GRRMHD simulations (Gammie, McKinney, and Tóth 2003; Tchekhovskoy, Narayan, and McKinney 2010; Tchekhovskoy, Narayan, and McKinney 2011; Sądowski et al. 2011; McKinney et al. 2014; Ryan, Dolence, and Gammie 2015; Ressler et al. 2015). Such studies have shown the connection between the accretion flow and the launching of jets, while others have been developed to specifically apply to investigate the features and radiation spectra of well-known sources of interest, such as Sgr A* and M87 (Mościbrodzka and Falcke 2013; Mościbrodzka et al. 2014; Mościbrodzka, Falcke, and Shiokawa 2016; Mościbrodzka et al. 2017; Ryan et al. 2017; Chael et al. 2018; Davelaar et al. 2018; Chael, Narayan, and Johnson 2019).

Along with theoretical considerations, MHD and GRMHD simulations are frequently paired with radiative transfer calculations in order to model the spectral energy distribution due to the radiative emission in the plasma - disc and jet. Non-thermal 
emission is commonly attributed to electrons being accelerated due to fast magnetic reconnection - see, e.g., de Gouveia dal Pino and Lazarian (2005), Giannios, Uzdensky, and Begelman (2009), Kowal, de Gouveia Dal Pino, and Lazarian (2011), Giannios (2013), de Gouveia Dal Pino and Kowal (2015), and Khiali and de Gouveia Dal Pino (2016). One way to verify this is by incorporating and tracking test particles and their energies upon being accelerated in fast reconnection sites (de Gouveia Dal Pino and Kowal 2015). More recently, electrons experiencing Fermi acceleration have been employed to explain observational features in the spectrum of 3C 273 (Lewis, Finke, and Becker 2018), including a recent flare (Lewis, Finke, and Becker 2019).

Along with subparsec MHD/GRMHD simulations of accretion and jets, another commonly used technique to investigate the origin of non-thermal emission is particlein-cell (PIC) simulations. In PIC simulations, particles are tracked in a Lagrangian frame, i.e., their positions and velocities are followed, while currents and densities are computed on Eulerian frames, i.e., on a fixed mesh. This is achieved by modeling the plasma as a collection of charged particles which are then moved upon integration of the Lorentz force. As these particles move, the currents they leave on the grid are used to compute the electromagnetic fields using Maxwell's equations. The new values of these EM fields on the grid are then passed to the particles, which are then moved by the Lorentz force, thus completing a cycle.

Many PIC studies have been employed, in both 2D and 3D, to study the formation of magnetic islands as well as mechanisms of particle acceleration and the origin of nonthermal emission in different contexts (Zenitani and Hoshino 2001; Drake et al. 2006; Cerutti et al. 2013; Sironi and Spitkovsky 2014; Kagan et al. 2015; Werner and Uzdensky 2017; Werner et al. 2018; Werner, Philippov, and Uzdensky 2019; Parfrey, Philippov, and Cerutti 2019) In Kowal, de Gouveia Dal Pino, and Lazarian (2011), it was shown that PIC results coincide with those of MHD plus test particles. In Sironi and Spitkovsky (2014), it was found that the particle spectrum over the entire reconnection region is 
that of a non-thermal power law with a slope harder than -2 for magnetizations $\sigma \gtrsim 10$, where the magnetization is related to the magnetic field $B_{0}$ and gas density $\rho$, being defined by

$$
\sigma \equiv \frac{B_{0}^{2}}{4 \pi \rho c^{2}}
$$

In Sironi and Spitkovsky (2014) - see Figure 5.1 - after magnetic reconnection starts from numerical noise, the reconnection layer breaks into a series of magnetic islands, also called plasmoids. The formation of plasmoids is due to the tearing instability: magnetic field lines decouple from the fluid, such that when two regions of opposite magnetic flux are brought into contact, plasmoids are formed. Over time, plasmoids will coalesce and grow to larger scales. In reality, 2D islands are cross-sections of 3D magnetic flux tubes.

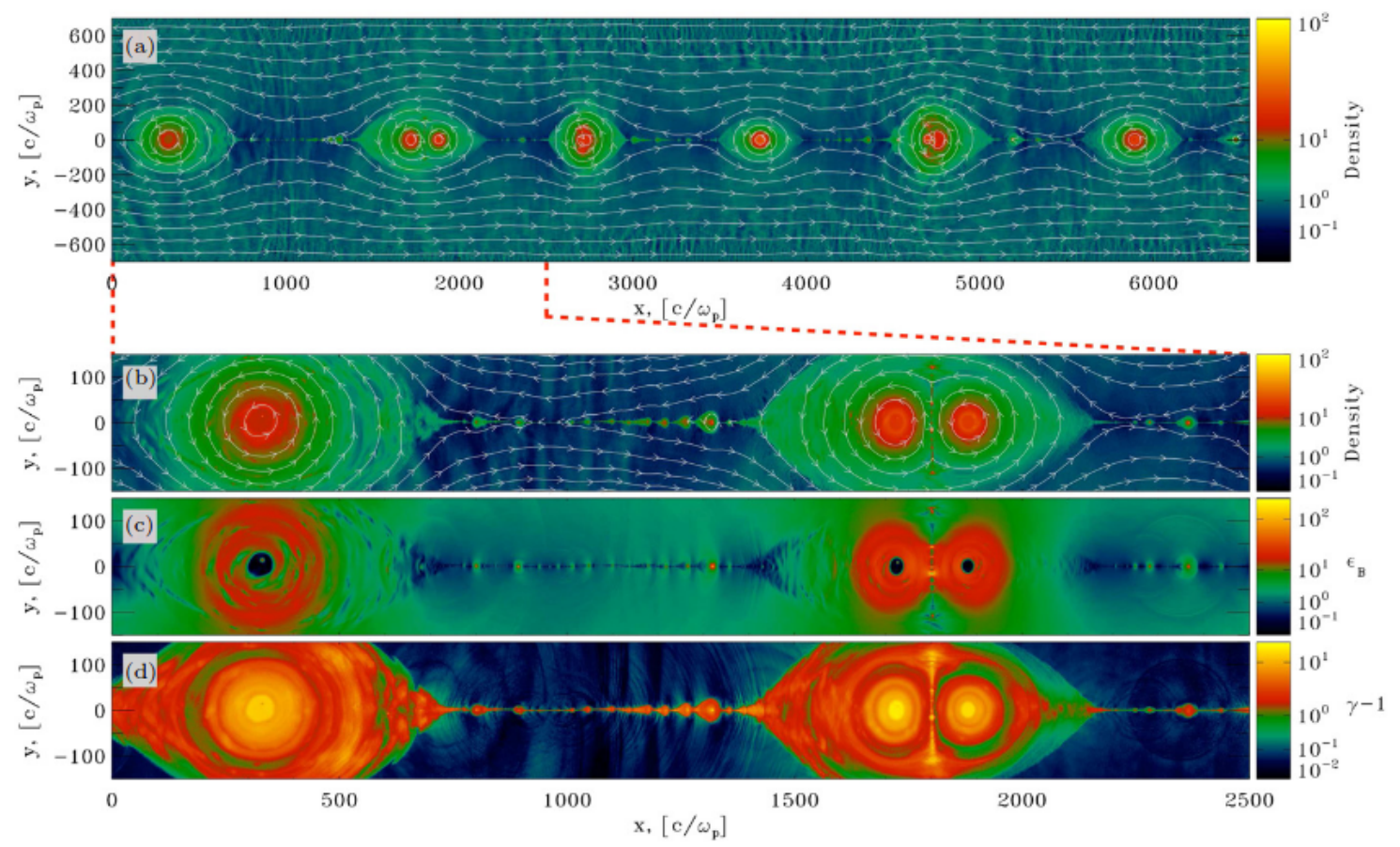

Figure 5.1: The top panel shows the particle density in a reconnection layer from a PIC simulation. The other three panels are zoomed-in plots of the region between $x=0$ and $x=2500$. They show, from top to bottom, the particle density, magnetic energy and mean kinetic energy per particle. Taken from Sironi and Spitkovsky (2014).

The dynamics of plasmoids is especially important in the wider context of current sheets. Current sheets are electric currents - hence characterized by high values of cur- 
rent density when compared to non-sheet regions - which form upon the annihilation of magnetic fields of opposing polarities, thus favoring the appearance of magnetic reconnection and particle acceleration. Upon entering the current sheet, the flow is advected towards the plasmoids by the reconnected magnetic field. All particles which are heated and accelerated by reconnection get trapped within the plasmoids, which concentrate most particles and energy. Inside the plasmoids, magnetic fields are compressed and therefore become stronger. Hence, it is expected that the bigger islands will dominate the synchrotron emission, as synchrotron power scales with $B_{0}^{2}$.

A series of papers added depth to the discussion of plasmoids as the sources of nonthermal emission, with applications to blazar jets (Sironi, Petropoulou, and Giannios 2015; Sironi, Giannios, and Petropoulou 2016; Petropoulou, Giannios, and Sironi 2016; Petropoulou et al. 2018; Christie et al. 2019a). Moreover, inspired by the findings in PIC simulations suggesting magnetic reconnection as an efficient mechanism for accelerating non-thermal electrons, Ball et al. (2016) and Ball et al. (2018) showed in GRMHD simulations the emergence of current sheets in the accretion disc of Sgr $A^{*}$, and argued that non-thermal electrons could explain the observed X-ray flares. More recent progress on the usage of GRMHD and particle transport aims to track and study the evolution of particles in larger scale simulations (Bacchini et al. 2019). Also, Parfrey, Philippov, and Cerutti (2019) implemented a general relativistic framework into PIC simulations of plasma in the black hole magnetosphere and demonstrated the extraction of energy from a rotating black hole, similar to Penrose (1969) and Blandford and Znajek (1977).

Here, we describe ongoing work in which we want to classify the current sheets regions in the disc and jet where magnetic reconnection is likely to occur and accelerate electrons - and also investigate the effects of changing the magnetic topology and black hole spin on the appearance and features of such sheets. 


\subsection{Simulations}

The simulations described in this work were carried out using HARMPI ${ }^{1}$, a threedimensional, MPI-parallelized version of HARM (Gammie, McKinney, and Tóth 2003; Noble et al. 2006). In our version of the code, we calculate the 4-current $J^{\mu}$ based on the algorithm present in the original HARM code, using the Maxwell Equations in covariant form,

$$
D_{v} F^{\mu v}=4 \pi J^{\mu}
$$

assuming symmetry with respect to the spatial coordinates.

One of our goals was to investigate the effects of changing the magnetic field configurations in the formation and appearance of current sheets in the jet. As a result, we ran simulations which differ from each other with respect to the configuration of the magnetic field topology in the accretion disc: two simulations evolve a MAD (magnetically arrested disc), while the other two evolve a SANE (standard and normal evolution).

In the MAD scenario, there is an accumulation of magnetic flux at the vicinity of the black hole. The accreting gas exerts pressure on the magnetic flux, which prevents it from escaping the innermost region of the accretion disc. In turn, this accumulated flux breaks down the accretion disc into smaller streams, causing a disruption in the accretion flow. In practice, to achieve a scenario in which the magnetic field lines accumulate in the black hole vicinity, GRMHD simulations often employ large torii with an initial magnetic field configuration consisting of a single poloidal loop that covers the entire torus. The net flux around the black hole remains at a high value and it accumulates at the inner parts of the disc. This initial setup is shown in Figure 5.2. The single loops are generated by

$$
A_{\phi}=\left(\frac{r}{r_{\mathrm{in}}}\right)^{3} \sin ^{3}(\theta) \frac{\rho_{\mathrm{av}}}{\rho_{\max }}-0.2
$$

\footnotetext{
${ }^{1}$ A public version of HARMPI is available at https://github. com/atchekho/harmpi
} 
where $\rho_{\text {av }}$ is a local average of $\rho$ and $\rho_{\max }$ is a normalization factor.

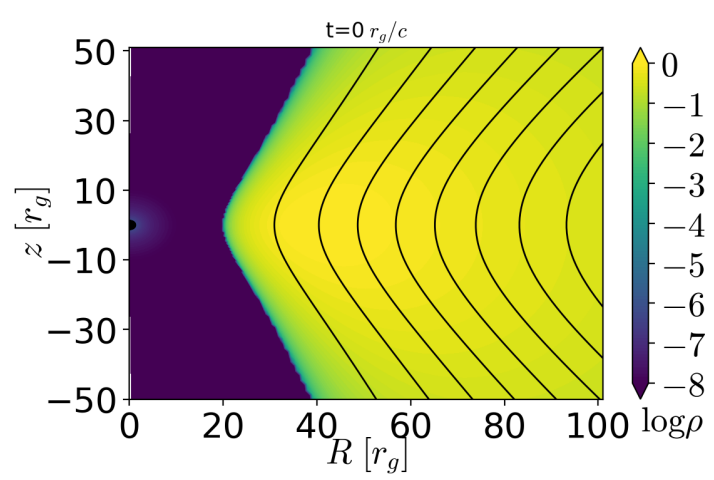

(a)

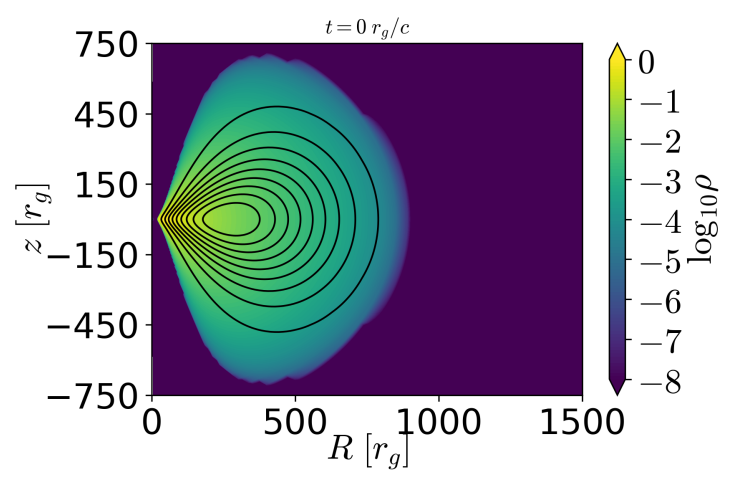

(b)

Figure 5.2: Initial configuration of the magnetic field topology (MAD) in simulation $2 \mathrm{dm} 9$, zoomed in (left) and out (right).

For our MAD scenarios, we ran two simulations where we varied the spin: $a=0.1$ and $a=0.9$. The torus follows a Fishbone and Moncrief (1976) prescription. The accretion disc's inner radius is $r_{\mathrm{in}}=20 r_{\mathrm{g}}$, where $r_{\mathrm{g}}=G M / c^{2}$ is the gravitational radius, $G$ is the gravitational constant, $M$ is the black hole mass and $c$ is the speed of light. The pressure maximum is at $r_{\max }=41 r_{\mathrm{g}}$. We choose a constant adiabatic index $\gamma_{\mathrm{ad}}=13 / 9$, corresponding to relativistic electrons and non-relativistic ions. The computational grid consists of $(t, r, \theta, \phi)$ in modified Kerr-Schild coordinates (Gammie, McKinney, and Tóth 2003; McKinney and Gammie 2004). Here, we show 2D simulations (i.e. a single $\phi$ cell) - we are currently running 3D simulations, which will be analyzed for the full study. The spatial grid resolution $N_{r} \times N_{\theta} \times N_{\phi}$ is $288 \times 192 \times 1$. We set the outer radial boundary at $r=10^{5} r_{\mathrm{g}}$. We carried out all simulations until $t_{\mathrm{f}}=36000 \mathrm{r}_{\mathrm{g}} / \mathrm{c}$.

In contrast, the SANE scenario does not show the accumulation of magnetic flux described above. In simulations, this is achieved by inserting poloidal loops of alternating polarity (see Figure 5.3). For our SANE, multi-loop simulation, we modeled the magnetic vector potential $A_{\mu}$ in a similar way as in Shafee, Narayan, and McClintock (2008), Penna et al. (2010), and McKinney, Tchekhovskoy, and Blandford (2012), implementing 
in HARMPI magnetic loops of alternating polarity which are generated by

$$
A_{\phi}=Q^{2} \sin \left[\frac{\log (r / S)}{\lambda_{\mathrm{B}}}\right]
$$

where $S=1.1 r_{\mathrm{in}}, \lambda_{\mathrm{B}}=0.09$. The quantity $Q$ is defined as

$$
Q=\left(\rho / \rho_{\max }-0.2\right)(r / M)^{3 / 4}
$$

but it is set to zero if $Q<0$ or $r<S$, with the gas density $\rho$ being normalized by the maximum gas density at the start of a run, $\rho_{\max }$.

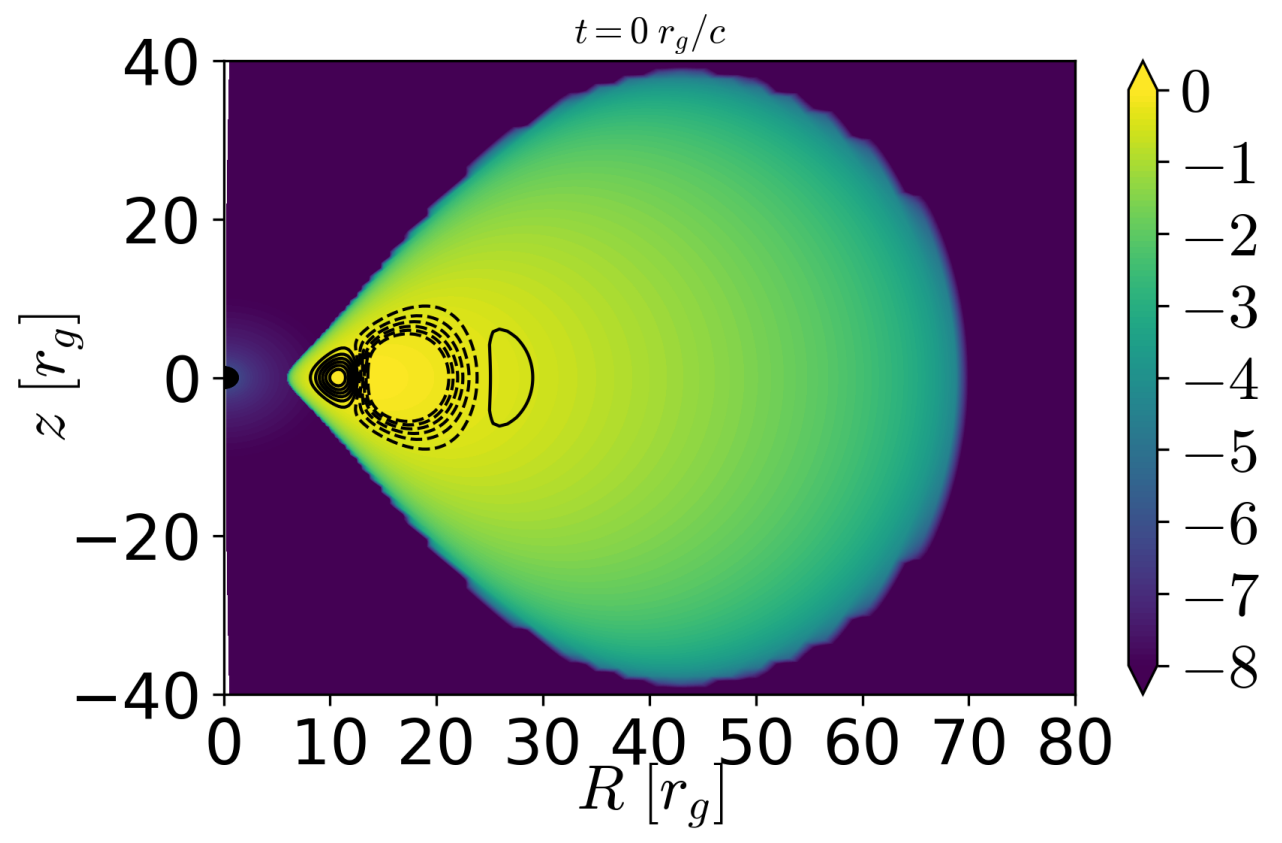

Figure 5.3: Initial configuration of the magnetic field topology (SANE) in simulation $2 \mathrm{ds} 9$.

As in the MAD scenarios, we ran simulations with $a=0.1$ and $a=0.9$, but here the accretion disc's inner radius is $r_{\mathrm{in}}=6 r_{\mathrm{g}}$, and the pressure maximum is at $r_{\max }=13 r_{\mathrm{g}}$. For theseSANE scenarios, we carried out the simulations until $t_{\mathrm{f}}=10000 \mathrm{r}_{\mathrm{g}} / \mathrm{c}$. Table 5.1 displays a summary of our simulation setup and parameters.

Recalling that blazars are classified into FSRQs - best described by thin discs where 
Table 5.1: Simulations considered and their parameters. The spatial resolution $\left(N_{r} \times\right.$ $\left.N_{\theta} \times N_{\phi}\right)$ is $288 \times 192 \times 1$.

\begin{tabular}{lccc}
\hline $\begin{array}{l}\text { Simulation } \\
\text { Name }\end{array}$ & $\begin{array}{c}\text { Magnetic Field } \\
\text { Topology }\end{array}$ & $a_{*}$ & $\begin{array}{c}t_{\text {final }} \\
r_{\mathrm{g}} / c\end{array}$ \\
\hline $2 \mathrm{dm} 1$ & MAD & 0.1 & $3.6 \times 10^{4}$ \\
$2 \mathrm{ds} 1$ & SANE & 0.1 & $10^{4}$ \\
$2 \mathrm{dm} 9$ & MAD & 0.9 & $3.6 \times 10^{4}$ \\
$2 \mathrm{ds} 9$ & SANE & 0.9 & $10^{4}$ \\
\hline
\end{tabular}

radiation is dynamically important - and BL Lacs, our simulations can only be applied to the latter case, as they do not take into account the dynamical effect of radiation.

\subsection{Comparison of simulations}

We focus our discussion in the simulations with rapidly rotating black holes, namely $2 \mathrm{dm} 9$ and $2 \mathrm{ds} 9$, since higher spin values lead to more powerful jets (Tchekhovskoy, Narayan, and McKinney 2011). In Figure 5.4, we show the toroidal component of the lab-frame magnetic field, $B_{\phi}$, at the same time in the the four simulations. We focus on the toroidal component because the alternating components appear more often in that direction. In Ball et al. (2018), the authors found that the $B_{\phi}$ appears to be more "twisted" in the SANE cases when compared to the MAD ones. However, it is difficult to devise a criterion to properly quantify this. At most, a qualitative analysis suggests that in some snapshots it does appear that the SANE fields are more twisted when compared to their MAD counterparts, but the converse is also true in some other cases. In a sense, this is not unexpected, given that MRI-induced turbulence occurs in all simulations. Moreover, due to construction, the SANE disc is expected to, at least initially, have more regions of alternating polarities. Still, a further analysis of this behavior is left for future work. 


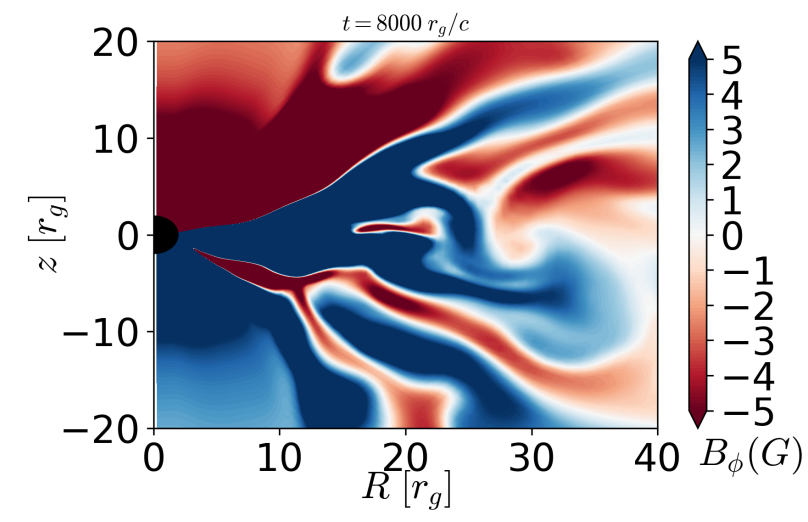

(a) $2 \mathrm{dm} 1$.

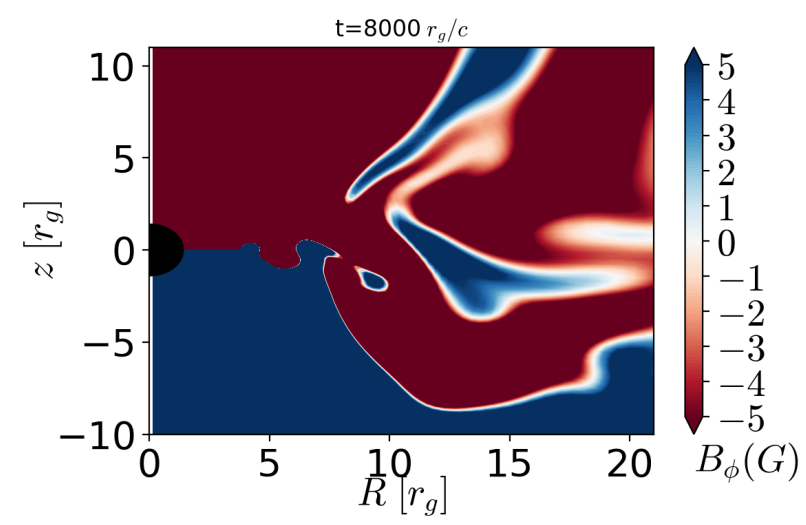

(c) $2 \mathrm{dm} 9$.

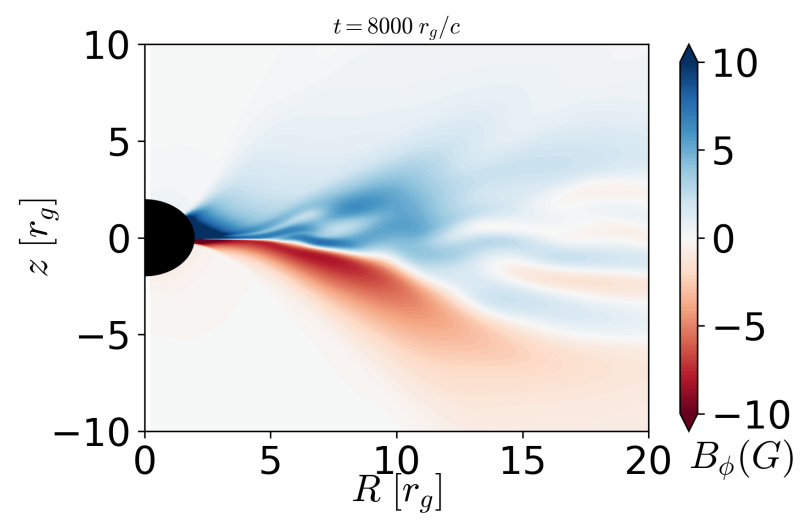

(b) $2 \mathrm{ds} 1$.

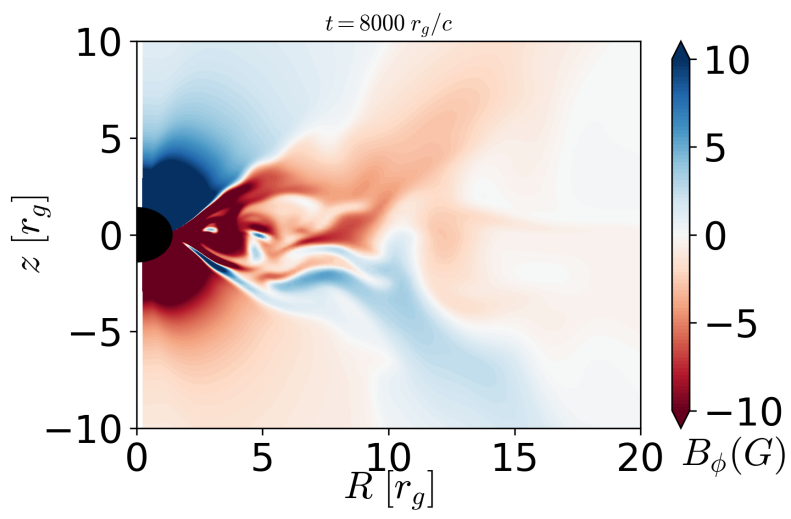

(d) 2 ds 9 .

Figure 5.4: Toroidal component of the lab-frame magnetic field, $B_{\phi}$, in four different simulations, all at the same time $t=8000 r_{\mathrm{g}} / c$. The torus is smaller for $a_{*}=0.1$ due to the Fishbone-Moncrief prescription.

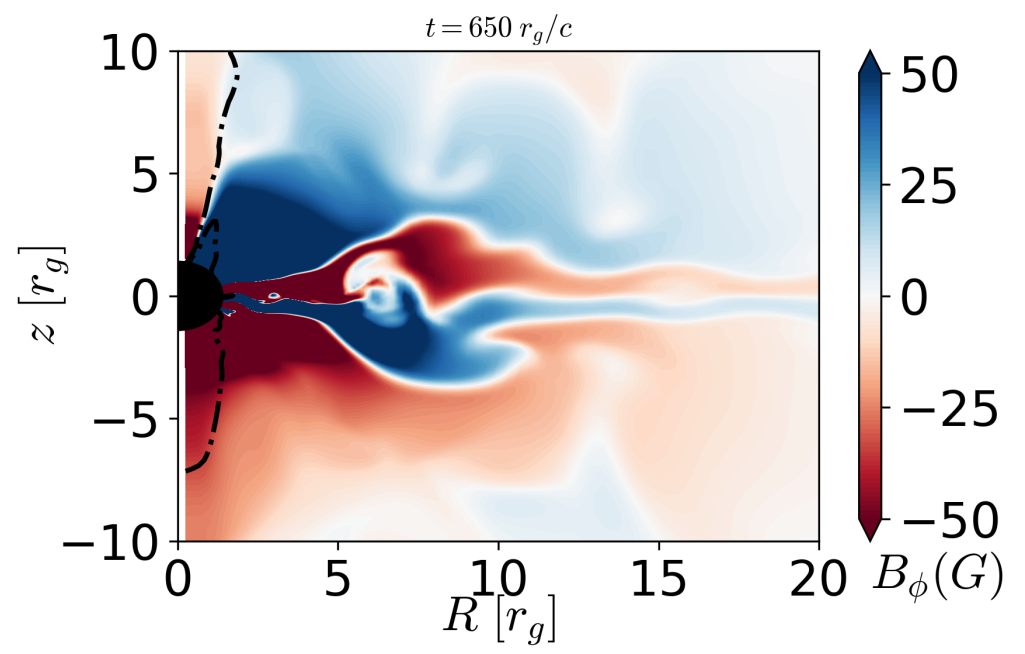

Figure 5.5: Toroidal component of the magnetic field $\left(B_{\phi}\right)$ in the lab frame in simulation 2ds9. The dash-dotted line corresponds to $b^{2} / \rho=1$ 
The SANE simulations are constructed in such a way that the opposing polarity fields will interact and thus lead to the appearance of current sheets, with some of them being accreted towards the black hole and later expelled from the black hole. This is clearly seen in Figure 5.5, where the black dash-dotted line corresponds to the boundary between funnel and disc. This boundary is set by $\sigma=b^{2} / \rho=1-$ we say that the region immediately around this threshold is the jet sheath. We thus notice a region of alternating polarity in $B_{\phi}$ at the jet sheath, and below we will show that a current sheet does form at that region.

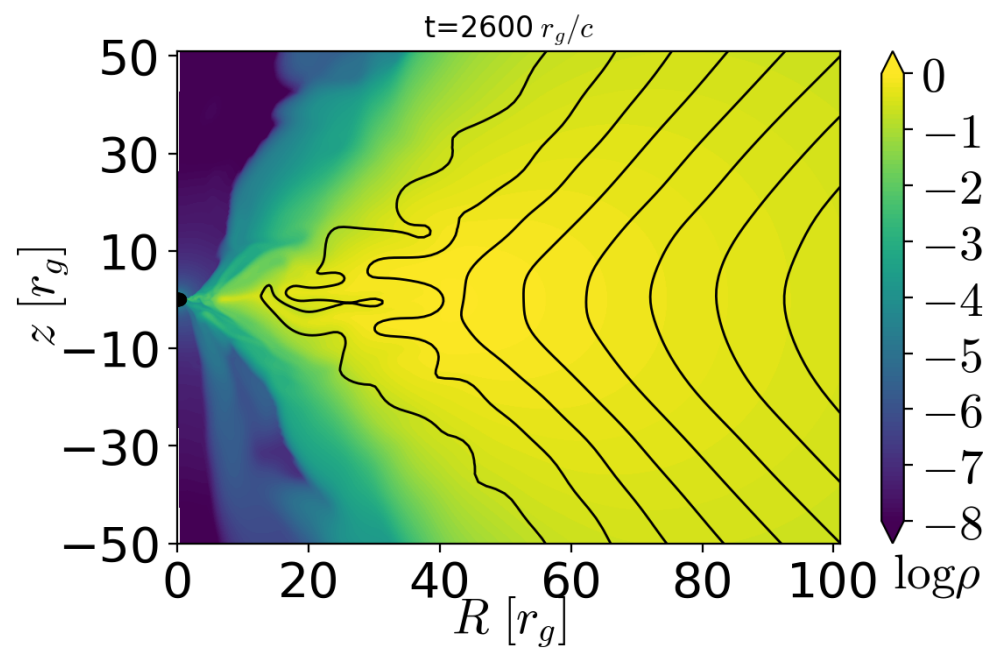

Figure 5.6: Gas reaches black hole at around $t=2600 r_{\mathrm{g}} / \mathrm{c}$ in simulation $2 \mathrm{dm} 9$.

For the MAD case, the larger accretion disc threaded by same-polarity magnetic field lines implied in accretion starting at a later time, with the gas reaching the black hole at around $t=2600 r_{\mathrm{g}} / c$ (Figure 5.6). Throughout the entire simulation, we also found regions of opposing fields, as exemplified in Figure 5.7.

More interestingly is that, once the flow achieves a MAD statem the jet occasionally gets choked due to the magnetic fields momentarily preventing the gas from reaching the black hole. When this happens, we see regions of opposing polarities in $B_{\phi}$ being dragged to the black hole and later being ejected away from it (Figure 5.8).

We noticed that varying the choice of magnetic field configuration also has an 


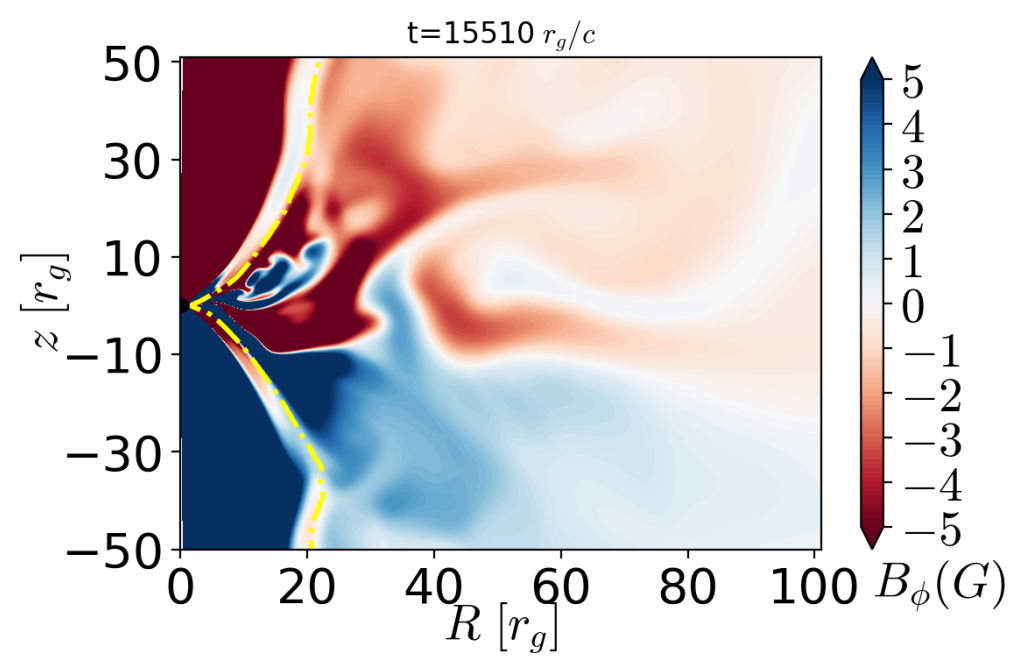

Figure 5.7: Toroidal component of the magnetic field $\left(B_{\phi}\right)$ in the lab frame in simulation $2 \mathrm{dm} 9$. The dash-dotted line corresponds to $b^{2} / \rho=1$

effect on the current sheets that appear on the jet. Initially, current sheets are more likely to appear in the SANE cases than in the MAD simulations. This is due to the alternating loops being accreted and then expelled from the black hole vicinity as outflows. However, since the loops are not large, this process is less proeminent as the simulation progresses. Larger loops will help in addressing this, but it is expected that regions of alternating polarity will interact with each other regardless of loop size. Also, longer simulation times and higher resolutions may be helpful in addressing this.

In the next section, we will describe the algorithm which has been used to explicitly identify the current sheets in our simulations.

\subsection{Identifying current sheets}

Our method for identifying regions in our snapshots that correspond to current sheets and potential reconnection regions is similar to other works, in particular Zhdankin et al. (2013), Ball et al. (2018), and Kadowaki, De Gouveia Dal Pino, and Stone (2018). As in Ball et al. (2018), since our resolution is small, we are unable to find islands (plasmoids) in our simulations. Here, reconnection sites appear as magnetic fields of 
opposing polarities meet, and the main components of the algorithm we use to identify and characterize these sites is briefly explained below. Further details can be found in the three aforementioned works.

The main idea of this algorithm stems from the fact that current sheets are regions of relatively high current values (compared to its adjacency - here taken to be the entire snapshot) located between magnetic fields of opposite polarity. In view of this, for each snapshot of our simulation, the algorithm goes through every grid cell and identifies those where the current $\sqrt{J^{\mu} J_{\mu}} \geq x J_{\text {mean, }}$ where $J_{\text {mean }}$ is the mean value of the current in the snapshot, and $x$ is a number to be determined by trial and error, but is usually 4 - 5 (Ball et al. 2018; Kadowaki, De Gouveia Dal Pino, and Stone 2018). Here, we set $x=4$. Then, the algorithm checks whether the magnetization at this cell is below a given threshold, $\sigma_{\mathrm{thr}}$, which we set here to $\sigma_{\mathrm{thr}}=10^{-4}$.

For each point $p$ identified following the steps described above, we call the current at such point $J_{\text {peak, }}$ then we check its immediately adjacent points. That is, for a $2 \mathrm{D}$ case, if the cell $(i, j)$ has been determined to contain a $J_{\text {peak, }}$ we check the adjacent cells given by $(i-1, j),(i+1, j),(i, j-1)$ and $(i, j+1)$.

If the current at an adjacent cell $p_{\text {adj }}$ is above $0.5 J_{\text {peak }}$ and the magnetization at $p_{\text {adj }}$ is smaller than $\sigma_{\mathrm{thr}}$, then we say that $p_{\text {adj }}$ is part of the same current sheet. Finally, for each adjacent point identified as being part of the same current sheet, we repeat the process, until the algorithm no longer finds any points that satisfy the criteria to be part of the same current sheet.

Another crucial point, not yet implemented here - see Zhdankin et al. (2013) and Kadowaki, De Gouveia Dal Pino, and Stone (2018) for details - is that at some sites the reconnection velocity might simply not be high. A consequence of this is that, since fast reconnection will not be present at some of these sites, the particles will not escape with high velocities, and the subsequent particle acceleration will not be efficient.

Before applying this algorithm to our simulation snapshots, we tested its validity 
in a series of "mock current sheets", in which we constructed regions where we knew, beforehand, that they would correspond to current sheets, and then applied the algorithm to check whether it correctly identified these regions. We did this by simply adding, by hand, a threshold value as well as a given value for the current in all cells of our "mock snapshot".

A very simple example of "mock" sheets is shown in Figure 5.9. The left panel displays our predetermined "mock current sheet", with the white cells being the analogous to the $J_{\text {peak }}$ cell: these are the cells with the highest values of current density. In the black cells the current was set to zero, for simplicity, while the colored cells around the white cells correspond to the sheet - the current density being higher than zero but lower than the maximum density at the white cells. A correct implementation of the algorithm should identify all of these cells. Indeed, this is the case, as shown in the right panel, which corresponds to the cells identified upon the application of our algorithm. We performed similar tests to different "mock current sheet" configurations, in order to guarantee the robustness of our algorithm. All tests produced similar results as the one in Figure 5.9.

Once the current sheets are identified following the algorithm described above, we turn to evaluating the magnetization around the current sheets. To do so, we must first find the direction which is perpendicular to the sheet. We do this by following an approach based on Ball et al. (2018).

For every point identified as belonging to a current sheet, we draw a box of width $S+1$ centered around this point. Next, we calculate the slope between the central point $p_{c}$ and every other point $p_{b}$ in the box that also belongs to the current sheet. The angle between these points is given by the inverse tangent of this slope. We average the angles between $p_{c}$ and every other point $p_{b}$, which gives us the mean angle between 
the central point and the other points, $\theta_{\text {mean }}$. Then, we calculate the mean slope using

$$
m_{\text {mean }}=\tan \theta_{\text {mean }}
$$

and the direction perpendicular to $m_{\text {mean }}$ is given by

$$
m=-\frac{1}{m_{\text {mean }}}
$$

Then, starting at the central point, we move along the line with coefficient $m$, selecting the points along the way and calculating the magnetization at these points. This process requires a few interpolations so that we arrive at a smoother profile for the desired quantities. As an example, Figure 5.10 compares $B_{\phi}$ with the current sheets identified by the algorithm. Not all regions in which $B_{\phi} \sim 0$ are identified as current sheets, since our criterion also takes into account the density $\rho$ in order to determine whether a current sheet is present or not - see Equation (5.1.1).

Another example is shown in Figure 5.11, which occurs in simulation 2dm9. There are a few points near the jet basis which have been identified as belonging to a current sheet. Yet, we don't see a continuation of said points as we move to the following snapshots, nor do we see such an appearance at larger $r_{\mathrm{g}}$, so it would be a bit premature to claim with full certainty that these points indeed correspond to current sheets. It might be that our thresholds for magnetization are over- or understimated, hence implying in fewer points being detected. Also, using magnetization as a criterion to determine which points belong to a current sheet might lead to a few imprecisions. This is due to GRMHD codes artificially injecting gas into the regions where density falls below a given threshold - such as the jet - as well as issues in calculating the magnetic field, which might lead to imprecisions in quantities which depend on it, such as magnetization. All these issues will be addressed by 3D simulations at higher resolutions. 


\subsection{Ongoing work and next steps}

With the goal of modeling blazar SEDs, radiative transfer models of analytic, semianalytic and numerical origin have been employed by several authors. For instance, in Petropoulou et al. (2018) and Christie et al. (2019a), the authors used a semi-analytical model (Mastichiadis and Kirk 1995) to estimate the emission in the reconnection plasmoids.

Another approach, which is often used in conjunction with GRMHD simulations, is to perform post-processing radiative transfer whereby one determines (usually by choosing threshold magnetization values) the regions in which magnetic reconnection might occur, leading to the acceleration of non-thermal electrons as found in Sironi and Spitkovsky (2014). In this approach, which has been used in Ball et al. (2018) and Davelaar et al. (2018), upon determining these reconnection regions, one injects non-thermal electrons following a given distribution.

Since magnetic reconnection can be a source of non-thermal electrons, one may pick threshold values for the plasma $\beta$, such that the non-thermal electrons may be injected in regions where $\beta<\beta_{\mathrm{thr}}$, since a small $\beta$ corresponds to higher magnetic pressure. In Li et al. (2015) and Ball et al. (2016), the authors chose $\beta_{\text {thr }}=0.2$, although other threshold values may be applicable.

It should be noted that radiative transfer calculations, which generally employ codes such as grmonty (Dolence et al. 2009) or GRay (Chan, Psaltis, and Özel 2013), are very sensitive to the effects of density floors. Recalling our discussion in Chapter 3, one might have nonphysical values of magnetic fields in regions of low gas density, such as the jets.

Consequently, it is customary to disregard the radiative contributions of regions where the magnetization $\sigma$ is larger than unity, which generally consist of the inner parts of the jets. However, as demonstrated in Chael, Narayan, and Johnson (2019), the related 
quantities may reach threshold values indicating saturation at magnetizations larger than unity. This motivates us not to exclude regions where $\sigma>1$. Instead, following Chael, Narayan, and Johnson (2019), we analyze a profile of the magnetization values along different radii - see Figure 5.12. We found a saturation value for the magnetizetion at $\sigma \approx 40-50$, which will allow us to adopt larger threshold values of magnetization when we perform radiative transfer calculations, thus excluding fewer cells from the analysis. Allowing for higher magnetization values will be useful in comparing our results with those of Christie et al. (2019a), who studied current sheets in different magnetizations $(\sigma=3,10,50)$.

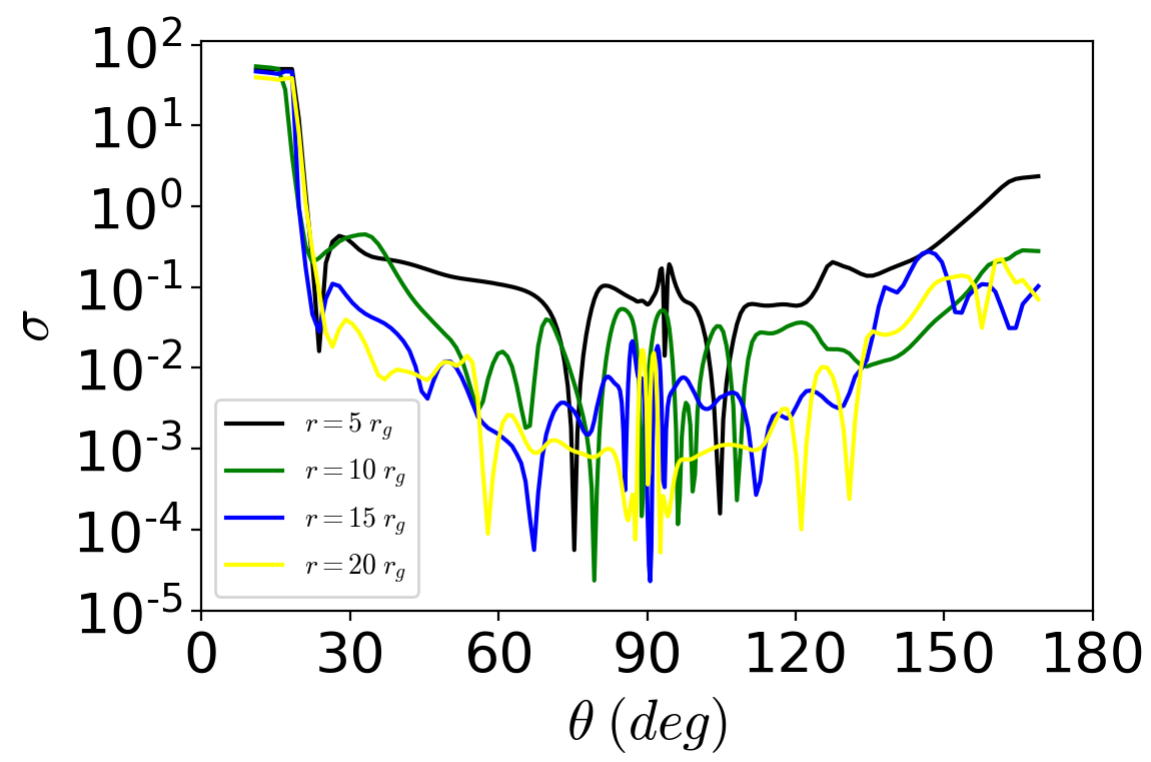

Figure 5.12: Magnetization $\sigma$ at four different radii (in $r_{\mathrm{g}}$ ): 5, 10, 15, 20. Saturation value for sigma is at around $\sigma=40-50$.

Based on our 2D results, we are running 3D simulations using the a similar parameter space as the 2D simulations described here, although we will also add another simulation with a toroidal initial magnetic field, which has been reported in Liska, Tchekhovskoy, and Quataert (2018) and Christie et al. (2019b) to lead to a dynamo-like behavior in the disc, generating flips in the magnetic fields and thus leading to the appearance of current sheets. Another feature of these simulations is that their resolutions are higher, which might be essential for the appearance and proper identification of the 
sheets. We will apply the same algorithms and methods used in the 2D simulations to these 3D simulations.

In these 3D simulations, we will also apply the radiative transfer post-processing code grmonty (Dolence et al. 2009) to generate non-thermal electrons in the regions of interest. We have modified grmonty so that it is able to read data from HARMPI. The modifications include a generalization to 3D simulations (the original grmonty only accounts for 2D simulations) as well as a grid adaptation. This grid adaptation is necessary because HARMPI grids contain newer features such as "cylindrification" close to the polar axis, which saves computational time (Tchekhovskoy, Narayan, and McKinney 2011), which has not been implemented in grmonty. This is because grmonty performs a series of calculations of the metric connection coefficients at each step of photon propagation. Performing these calculations analytically - which is the case of the MKS coordinate system in grmomty - is relatively quick, but cylindrified coordinates require numerical derivatives. These frequent calculations lead to a significant reduction in code performance - see, for instance, Ressler et al. (2017). Hence, it is easier to leave the grmomty grid unaltered and perform a regrid and interpolation of quantities from the HARMPI simulations into the grmonty grid.

Moreover, taking into account our discussion on magnetization threshold, we will be guided by magnetization profiles similar to Figure 5.12 to select points in the jet and/or sheath where the magnetization is lower than a threshold value to be determined, but guaranteed to be higher than unity. This will allow us to give a proper characterization of the regions surrounding the current sheets, so that we may compare our radiative transfer results to other works, such as Christie et al. (2019a). 


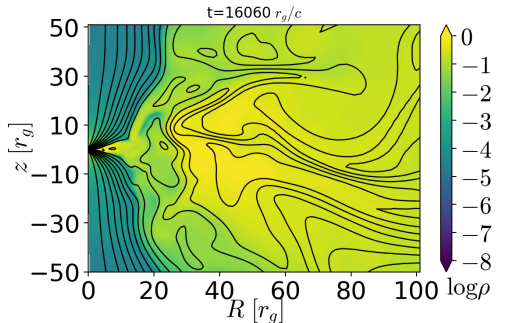

(a)

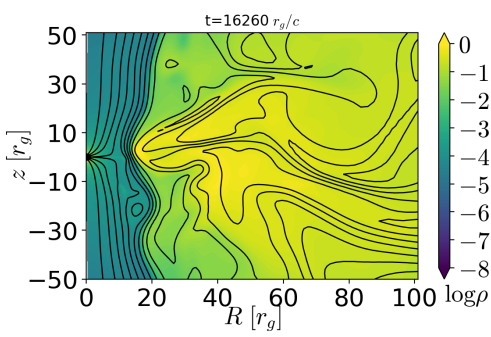

(c)

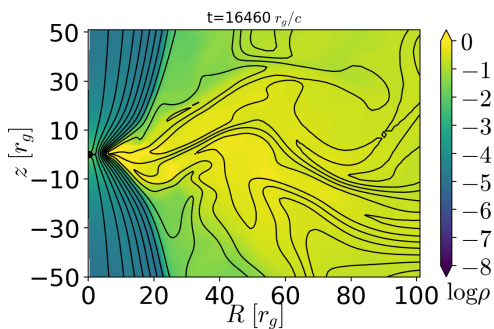

(e)

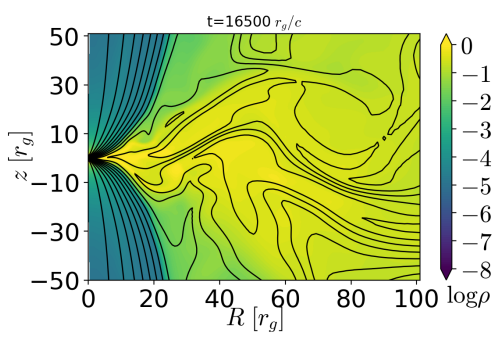

(g)

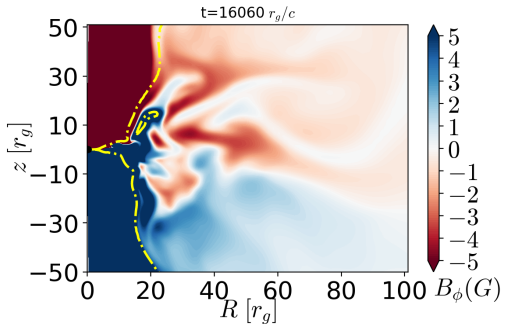

(b)

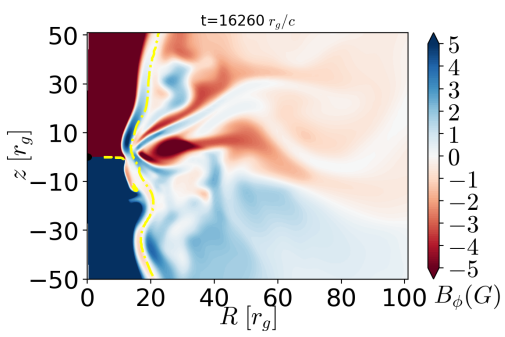

(d)

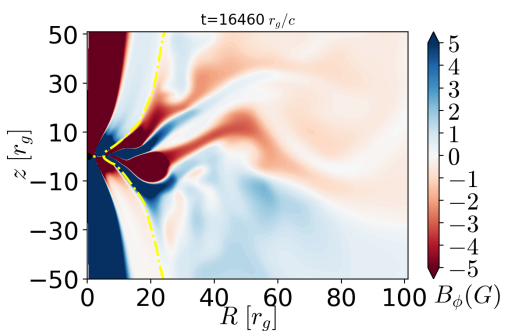

(f)

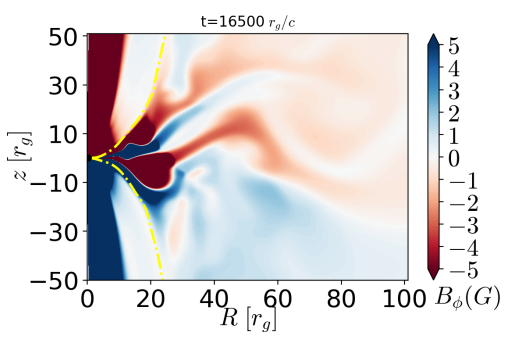

(h)

Figure 5.8: Left: gas density. Right: toroidal component of the lab-frame magnetic field, $B_{\phi}$, in simulation $2 \mathrm{dm} 9$. The gas is sometimes prevented from reaching the black hole due to the magnetic fields accumulating at its vicinity. This behavior occurred multiple times throughout the simulation. 

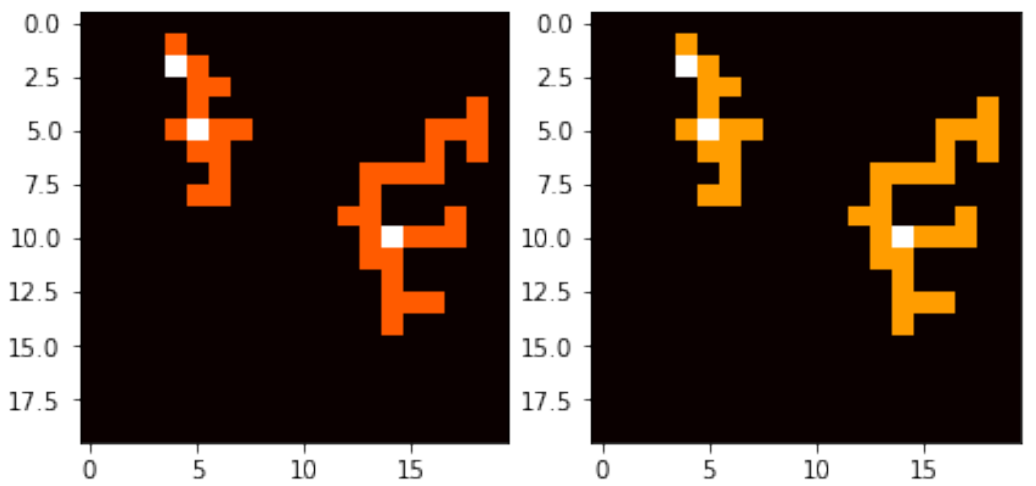

Figure 5.9: Left panel: mock current sheets, where we predetermined the values of the current in each cell. The white cells corresponds to the equivalent of $J_{\text {peak, }}$, the maximum value of the current in the snapshot. The colored cells correspond to the current sheets. Right panel: current sheets found by the algorithm corresponds to the predetermined sheet, suggesting the algorithm correctly identifies the current sheets. White and colored cells same as in left panel. The $\mathrm{x}$ and $\mathrm{y}$ axes simply display the spatial position and are of no physical interest. 


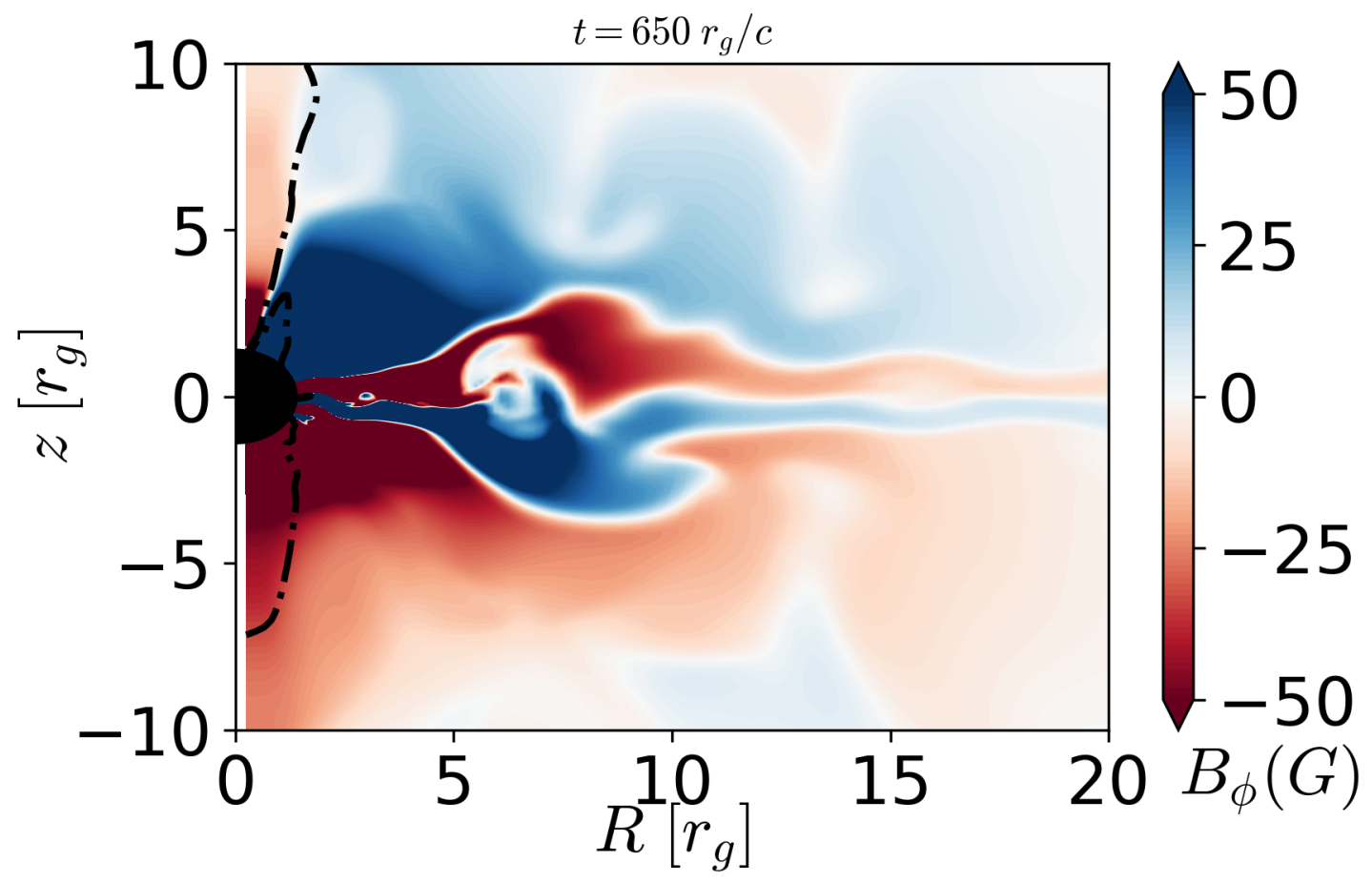

(a) Toroidal component of the magnetic field $\left(B_{\phi}\right)$ in the lab frame. This is the same as Figure 5.5

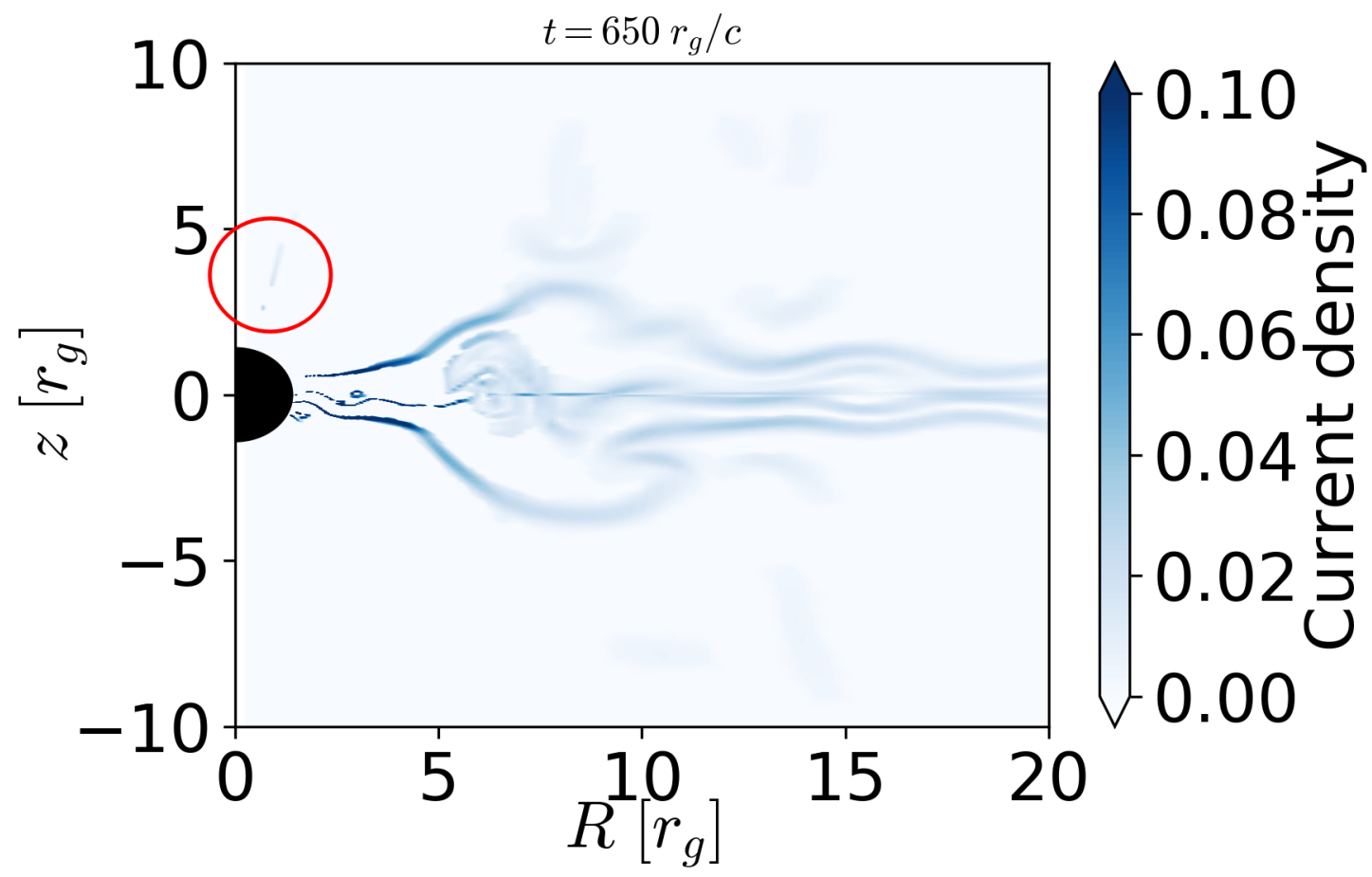

(b) Current sheets. Current density values set to zero for points not belonging to sheet.

Figure 5.10: Simulation 2ds9. Toroidal magnetic field and current sheets in the accretion disc and near the jet basis (circled in red), as identified by the algorithm. Sheets are identified using the magnetization in the toroidal direction. 


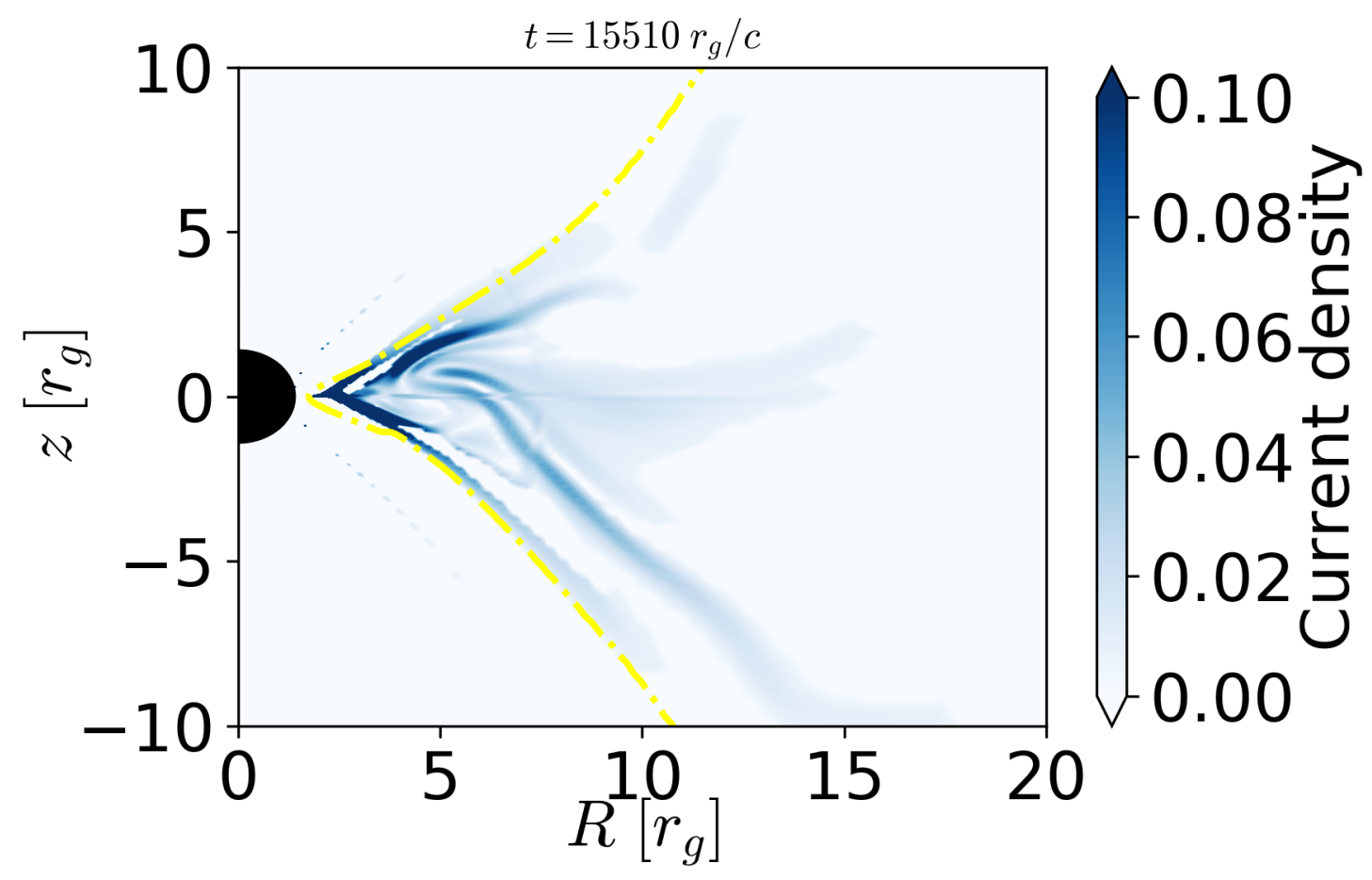

Figure 5.11: Simulation 2dm9. Current sheets in the accretion disc and near the jet basis, as identified by the algorithm. Sheets are identified using the magnetization in the toroidal direction. 


\section{Chapter 6}

\section{Conclusions and future directions}

In this thesis, our main topic of study was blazars. We performed two different types of studies, both aimed at answering fundamental questions related to blazars.

In our first study, we used $\gamma$-ray data to calculate the jet efficiency of FSRQs. We then proceeded to test the accuracy of theoretical predictions for the black hole spins in these objects. We found an average efficiency of about $10 \%$ for these objects. Using models that relate the BH spin to the jet effciency based on GRMHD simulations, we estimate the BH spins of the population of FSRQ blazars. We found a mean spin of $a_{*}=0.84_{-0.25}^{+0.11}$, with a lower limit estimated at $a_{*}^{\text {lower }}=0.59$. Our spin values are consistent with expectarions from merger-driven scenarios of SMBH evolution, although four objects in our sample could not have their spins determined, as they had efficiencies larger than the maximal efficiency that our model supported. This suggests that either the accretion rates of these four objects may be higher than assumed, their discs are thicker, or the jet model is not appropriate to describe these objects. Currently, we are investigating the luminosities of the sample of FSRQs at other wavelengths, in order to provide a better explanation for the possible correlation we found between SMBH mass and jet luminosities.

This study, presented in Chapter 4, focused only on FSRQs, which are modeled by thin, radiatively efficient discs. A natural, complementary study will focus on BL Lacs, 
best described by geometrically thick, optically thin discs. Previous works, such as Plotkin et al. (2011), used Sloan Digital Sky Survey (SDSS) observations to estimate the masses of the central SMBH in BL Lacs, and will be useful when used in conjunction with Fermi-LAT observations of $\gamma$-ray luminosities of BL Lacs, allowing us to study this class of objects in a similar way as we did with FSRQs. Combined, these two studies will provide a more complete comparison between simulation-based models and observations of blazars. We also plan to investigate the BL Lac objects in other wavelenghts, with the goal of providing a wider picture of $\mathrm{SMBH}$ masses, spins and luminosities.

Our second study, which is underway and was presented in Chapter 5, consists of GRMHD simulations of RIAFs which can be applied to BL Lacs. Our goal is to understand a fundamental aspect of black hole jets: where and how does the non-thermal emission in blazars originate? For this, we want to precisely model the jet regions in which non-thermal emission may occur. Using 2D simulations as a testing ground, we applied an algorithm that identifies current sheets in the accretion disc and jet, and we are applying the same method to 3D simulations. The next step is to perform radiative transfer calculations using grmonty with the goal of injecting non-thermal electrons (Pandya et al. 2016; Davelaar et al. 2018) in the regions identified by our algorithm as sources of non-thermal emission. We will then compare these results with semianalytic models such as Christie et al. (2019a), although our methods can be applied to other AGN classes and even black hole binaries.

The simulations presented here have a few limitations. For instance, they do not evolve the photon field and do not treat the momentum transferred between radiation and the gas, which limits our code to RIAFs only. A natural next step is to employ codes that are capable of evolving radiatively efficient accretion flows. Alternatively, an ad hoc implementation of radiative cooling, as in Avara, McKinney, and Reynolds (2016), may be helpful. We are assessing both possibilities. 
We will apply such a code to a series of astrophysical cases, which include Sgr A* and $\mathrm{M} 87$, in view of the recent obtention of an image of $M 87^{*}$ as well as future efforts by the EHT collaboration to image and precisely model these two systems. Still, there are many other cases of interest which we plan to study. In particular, the SEDs and light curves which we plan to obtain with this modified code will be employed to study variability across multiple wavelengths, which is a common feature of many AGN. We plan to study the effect of non-thermal emission on variability. 


\section{Bibliography}

Abbott, B. P. et al. "Gravitational Waves and Gamma-Rays from a Binary Neutron Star Merger: GW170817 and GRB 170817A". In: The Astrophysical Journal 848.2 (2017), p. L13. Dor: 10. 3847/2041-8213/aa920C. URL: https://doi . org/10. 3847\%2F2041$8213 \% 2$ Faa920c.

Abbott, B. P. et al. “GW170817: Observation of Gravitational Waves from a Binary Neutron Star Inspiral". In: Phys. Rev. Lett. 119 (16 2017), p. 161101. Dor: 10 . 1103 / PhysRevLett.119.161101. uRL: https://link.aps.org/doi/10.1103/PhysRevLett. 119.161101.

Abbott, B. P. et al. "Observation of Gravitational Waves from a Binary Black Hole Merger". In: Phys. Rev. Lett. 116 (6 2016), p. 061102. Dor: 10.1103/PhysRevLett. 116.061102. URL: http://link. aps.org/doi/10.1103/PhysRevLett.116.061102.

Abdo, A. A. et al. "A change in the optical polarization associated with a $\gamma$-ray flare in the blazar 3C279". In: Nature 463 (Feb. 2010), pp. 919-923. Dor: 10 . 1038/nature08841. arXiv: 1004.3828 [astro-ph.C0].

Abramowicz, M. A. and P. C. Fragile. "Foundations of Black Hole Accretion Disk Theory". In: Living Reviews in Relativity 16 (Jan. 2013), p. 1. arXiv: 1104. 5499 [astro-ph.HE].

Abramowicz, M. A. et al. "Slim accretion disks". In: ApJ 332 (Sept. 1988), pp. 646-658. Dor: $10.1086 / 166683$. 
Acero, F. et al. “Fermi Large Area Telescope Third Source Catalog”. In: ApJS 218, 23 (June 2015), p. 23. DOI: 10.1088/0067-0049/218/2/23. arXiv: 1501.02003 [astro-ph.HE].

Ackermann, M. et al. "Fermi-LAT Observations of the Gamma-Ray Burst GRB 130427A". In: Science 343.6166 (Jan. 2014), pp. 42-47. Dor: 10.1126/science. 1242353. arXiv: 1311.5623 [astro-ph.HE].

Ackermann, M. et al. “Minute-timescale \&gt;100 MeV $\gamma$-Ray Variability during the Giant Outburst of Quasar 3C 279 Observed by Fermi-LAT in 2015 June". In: ApJL 824.2, L20 (June 2016), p. L20. DoI: 10. 3847 /2041-8205/824/2/L20. arXiv: 1605.05324 [astro-ph.HE].

Ackermann, M. et al. “Multiwavelength Evidence for Quasi-periodic Modulation in the Gamma-Ray Blazar PG 1553+113". In: ApJL 813, L41 (Nov. 2015), p. L41. DoI: 10. 1088/2041-8205/813/2/L41.

Ackermann, M. et al. “The Second Catalog of Active Galactic Nuclei Detected by the Fermi Large Area Telescope". In: ApJ 743, 171 (Dec. 2011), p. 171. Dor: 10. 1088/0004637X/743/2/171. arXiv: 1108.1420 [astro-ph.HE].

Ackermann, M. et al. "The Spectrum and Morphology of the Fermi Bubbles". In: ApJ 793.1, 64 (Sept. 2014), p. 64. Dor: 10.1088/0004-637X/793/1/64. arXiv: 1407.7905 [astro-ph.HE].

Ajello, M. et al. "The Cosmic Evolution of Fermi BL Lacertae Objects". In: ApJ 780.1, 73 (Jan. 2014), p. 73. DoI: $10.1088 / 0004-637 X / 780 / 1 / 73$. arXiv: 1310.0006 [astro-ph.c0].

Akritas, M. G. and M. A. Bershady. "Linear Regression for Astronomical Data with Measurement Errors and Intrinsic Scatter". In: ApJ 470 (Oct. 1996), p. 706. DoI: 10. 1086/177901. eprint: arXiv: astro-ph/9605002.

Akritas, M. G. and J. Siebert. “A test for partial correlation with censored astronomical data". In: MNRAS 278 (Feb. 1996), pp. 919-924. eprint: arXiv: astro-ph/9508018. 
Anile, A. M. Relativistic Fluids and Magneto-Fluids. 1st. Cambridge Monographs on Mathematical Physics. Cambridge University Press, 1989.

Atwood, W. B. et al. "The Large Area Telescope on the Fermi Gamma-Ray Space Telescope Mission". In: ApJ 697 (June 2009), pp. 1071-1102. Dor: 10 . 1088/0004637X/697/2/1071. arXiv: 0902.1089 [astro-ph. IM].

Avara, M. J., J. C. McKinney, and C. S. Reynolds. "Efficiency of thin magnetically arrested discs around black holes". In: MNRAS 462.1 (July 2016), pp. 636-648. ISSN: 0035-8711. DOI: 10.1093/mnras/stw1643. eprint: http: //oup . prod.sis. lan/mnras/articlepdf/462/1/636/18755776/stw1643 . pdf. uRL: https: //doi . org/10 . 1093/mnras / stw1643.

Bacchini, F. et al. "Generalized, Energy-conserving Numerical Simulations of Particles in General Relativity. II. Test Particles in Electromagnetic Fields and GRMHD”. In: ApJS 240.2, 40 (2019), p. 40. Dor: 10.3847/1538-4365/aafcb3. arXiv: 1810.00842 [astro-ph.HE].

Balbus, S. A. and J. F. Hawley. “A powerful local shear instability in weakly magnetized disks. I - Linear analysis. II - Nonlinear evolution". In: ApJ 376 (July 1991), pp. 214233. DOI: $10.1086 / 170270$.

— "Instability, turbulence, and enhanced transport in accretion disks". In: Reviews of Modern Physics 70 (Jan. 1998), pp. 1-53. DoI: 10.1103/RevModPhys . 70.1.

Ball, D. et al. "Particle Acceleration and the Origin of X-Ray Flares in GRMHD Simulations of SGR A". In: ApJ 826.1, 77 (2016), p. 77. DOI: 10. 3847/0004-637X/826/1/77. arXiv: 1602.05968 [astro-ph.HE].

Ball, D. et al. "The Properties of Reconnection Current Sheets in GRMHD Simulations of Radiatively Inefficient Accretion Flows". In: ApJ 853.2, 184 (2018), p. 184. DoI: 10.3847/1538-4357/aaa42f. arXiv: 1705.06293 [astro-ph.HE].

Bally, J. "Protostellar Outflows". In: ARAEA 54 (2016), pp. 491-528. Dor: 10 . 1146 / annurev-astro-081915-023341. 
Baloković, M. et al. "Multiwavelength Study of Quiescent States of Mrk 421 with Unprecedented Hard X-Ray Coverage Provided by NuSTAR in 2013". In: ApJ 819.2, 156 (2016), p. 156. DOI: 10. $3847 / 0004-637 \mathrm{X} / 819 / 2 / 156$. arXiv: 1512.02235 [astro-ph.HE].

Barthel, P. D. et al. “New Superluminal Quasar 1633+382 and the Blazar-Gamma-Ray Connection". In: ApJL 444 (1995), p. L21. Dor: 10. 1086/187850.

Bignami, G. F. et al. “3C 273 revisited : confirmation by COS-B of high energy gamma-ray emission." In: AEA 93 (1981), pp. 71-75.

Blandford, R. D. and R. L. Znajek. “Electromagnetic extraction of energy from Kerr black holes". In: MNRAS 179 (May 1977), pp. 433-456.

Blandford, R., D. Meier, and A. Readhead. “Relativistic Jets from Active Galactic Nuclei". In: ARAEA 57 (2019), pp. 467-509. Dor: 10 . 1146/annurev-astro-081817Q51948. arXiv: 1812.06025 [astro-ph.HE].

Böttcher, M. and C. D. Dermer. “An Evolutionary Scenario for Blazar Unification”. In: The Astrophysical Journal 564.1 (2002), pp. 86-91. DOI: 10. 1086/324134. URL: https: //doi .org/10.1086\%2F324134.

Böttcher, M. et al. "Leptonic and Hadronic Modeling of Fermi-detected Blazars". In: ApJ 768.1, 54 (2013), p. 54. DOI: 10.1088/0004-637X/768/1/54. arXiv: 1304.0605 [astro-ph.HE].

Bridle, A. H. and R. A. Perley. “Extragalactic Radio Jets”. In: ARAEA 22 (1984), pp. 319358. Dor: 10.1146/annurev . aa.22.090184.001535.

Cavagnolo, K. W. et al. "A Relationship Between AGN Jet Power and Radio Power". In: ApJ 720 (Sept. 2010), pp. 1066-1072. DoI: 10.1088/0004-637X/720/2/1066. arXiv: 1006.5699 [astro-ph.CO].

Cavaliere, A. and V. D’Elia. “The Blazar Main Sequence”. In: The Astrophysical Journal 571.1 (2002), pp. 226-233. DOI: 10 . 1086/339778. uRL: https : / / doi . org/10 . 1086\% 2F339778. 
Cerutti, B. et al. "Simulations of Particle Acceleration beyond the Classical Synchrotron Burnoff Limit in Magnetic Reconnection: An Explanation of the Crab Flares". In: ApJ 770.2, 147 (June 2013), p. 147. Dor: 10.1088/0004-637X/770/2/147. arXiv: 1302.6247 [astro-ph.HE].

Chael, A., R. Narayan, and M. D. Johnson. “Two-temperature, Magnetically Arrested Disc simulations of the jet from the supermassive black hole in M87". In: MNRAS 486.2 (2019), pp. 2873-2895. DoI: 10.1093/mnras/stz988. arXiv: 1810.01983 [astro-ph.HE].

Chael, A. et al. "The role of electron heating physics in images and variability of the Galactic Centre black hole Sagittarius A*”. In: MNRAS 478.4 (2018), pp. 5209-5229. DoI: 10.1093/mnras/sty1261. arXiv: 1804.06416 [astro-ph.HE].

Chael, A. A., R. Narayan, and A. Saowski. "Evolving non-thermal electrons in simulations of black hole accretion". In: MNRAS 470.2 (2017), pp. 2367-2386. Dor: 10.1093/mnras/stx1345. arXiv: 1704.05092 [astro-ph.HE].

Chan, C.-k., D. Psaltis, and F. Özel. “GRay: A Massively Parallel GPU-based Code for Ray Tracing in Relativistic Spacetimes". In: ApJ 777, 13 (Nov. 2013), p. 13. DoI: 10.1088/0004-637X/777/1/13. arXiv: 1303.5057 [astro-ph. IM].

Chandrasekhar, S. “The Maximum Mass of Ideal White Dwarfs”. In: ApJ 74 (1931), p. 81. DOI: $10.1086 / 143324$.

Christie, I. M. et al. “Radiative signatures of plasmoid-dominated reconnection in blazar jets". In: MNRAS 482.1 (2019), pp. 65-82. DoI: 10.1093 /mnras / sty2636. arXiv: 1807.08041 [astro-ph.HE].

Christie, I. M. et al. "The role of magnetic field geometry in the evolution of neutron star merger accretion discs". In: MNRAS 490.4 (2019), pp. 4811-4825. DoI: 10. 1093 / mnras/stz2552. arXiv: 1907.02079 [astro-ph.HE].

Contopoulos, I., D. Gabuzda, and N. Kylafis. The Formation and Disruption of Black Hole Jets. Astrophysics and Space Science Library. Springer International Publishing, 
2014. ISBN: 9783319103563. uRL: https : / / books . google . com . br / books ? id= 3WFNBQAAQBAJ.

D'Abrusco, R. et al. “The WISE Blazar-like Radio-loud Sources: An All-sky Catalog of Candidate -ray Blazars". In: The Astrophysical Journal Supplement Series 215.1 (2014), p. 14. URL: http: //stacks. iop.org/0067-0049/215/i=1/a=14.

Davelaar, J. et al. “General relativistic magnetohydrodynamical $\kappa$-jet models for Sagittarius A*". In: AEA 612, A34 (2018), A34. DOI: 10 . 1051/0004-6361/201732025. arXiv: 1712.02266 [astro-ph.HE].

de Gouveia dal Pino, E. M. and A. Lazarian. “Production of the large scale superluminal ejections of the microquasar GRS 1915+105 by violent magnetic reconnection". In: AEA 441 (Oct. 2005), pp. 845-853. Dor: 10.1051/0004-6361:20042590.

de Gouveia Dal Pino, E. M. and G. Kowal. “Particle Acceleration by Magnetic Reconnection". In: Magnetic Fields in Diffuse Media. Ed. by A. Lazarian, E. M. de Gouveia Dal Pino, and C. Melioli. Vol. 407. Astrophysics and Space Science Library. 2015, p. 373. DOI: $10.1007 / 978-3-662-44625-6 \_13$.

Dexter, J. “A public code for general relativistic, polarised radiative transfer around spinning black holes". In: MNRAS 462.1 (2016), pp. 115-136. Dor: 10 . 1093/mnras/ stw1526. arXiv: 1602.03184 [astro-ph.HE].

Dolence, J. C. et al. "grmonty: A Monte Carlo Code for Relativistic Radiative Transport". In: ApJS 184.2 (2009), pp. 387-397. DOI: 10 . 1088/0067-0049/184/2/387. arXiv: 0909.0708 [astro-ph.HE].

Drake, J. F. et al. "Formation of secondary islands during magnetic reconnection". In: 33.13, L13105 (July 2006), p. L13105. DOI: 10. 1029/2006GL025957.

Dubois, Y., M. Volonteri, and J. Silk. “Black hole evolution - III. Statistical properties of mass growth and spin evolution using large-scale hydrodynamical cosmological simulations". In: MNRAS 440 (May 2014), pp. 1590-1606. Dor: 10.1093/mnras / stu373. arXiv: 1304.4583 [astro-ph.C0]. 
Eisenhauer, F. et al. "SINFONI in the Galactic Center: Young Stars and Infrared Flares in the Central Light-Month". In: The Astrophysical Journal 628.1 (2005), pp. 246-259. DOI: $10.1086 / 430667$. uRL: https: //doi .org/10.1086\%2F430667.

Fabian, A. C. "Observational Evidence of Active Galactic Nuclei Feedback". In: $A R A \mathcal{E} A$ 50 (Sept. 2012), pp. 455-489. Dor: 10. 1146/annurev-astro-081811-125521. arXiv: 1204.4114 [astro-ph.C0].

Fan, X. et al. "The Discovery of a Gravitationally Lensed Quasar at $\mathrm{z}=6.51$ ”. In: ApJL 870.2, L11 (2019), p. L11. DoI: 10 . 3847 / 2041 - 8213/aaeffe. arXiv: 1810 . 11924 [astro-ph.GA].

Fanaroff, B. L. and J. M. Riley. “The Morphology of Extragalactic Radio Sources of High and Low Luminosity". In: Monthly Notices of the Royal Astronomical Society 167.1 (Apr. 1974), 31P-36P. IssN: 0035-8711. DoI: 10. 1093/mnras/167 . 1 . 31P. eprint: http: //oup . prod.sis. lan/mnras/article-pdf/167/1/31P/8079923/mnras167031P.pdf. URL: https: //doi .org/10.1093/mnras/167.1.31P.

Fender, R. P., J. Homan, and T. M. Belloni. “Jets from black hole X-ray binaries: testing, refining and extending empirical models for the coupling to X-rays". In: MNRAS 396 (July 2009), pp. 1370-1382. Dor: 10.1111/ j .1365-2966 . 2009 . 14841 . x. arXiv: 0903.5166 [astro-ph.HE].

Fishbone, L. G. and V. Moncrief. "Relativistic fluid disks in orbit around Kerr black holes." In: ApJ 207 (1976), pp. 962-976. Dor: 10. 1086/154565.

Fossati, G. et al. "A unifying view of the spectral energy distributions of blazars". In: MNRAS 299 (Sept. 1998), pp. 433-448. Dor: 10.1046/ j . 1365-8711. 1998.01828 . x. eprint: arXiv: astro-ph/9804103.

Frank, J., A. King, and D. J. Raine. Accretion Power in Astrophysics. 3rd. Cambridge University Press, Jan. 2002. 
Gallo, E. "Radio Emission and Jets from Microquasars". In: The Jet Paradigm, Lecture Notes in Physics, Volume 794. ISBN 978-3-540-76936-1. Springer-Verlag Berlin Heidelberg, 2010, p. 85. Ed. by T. Belloni. Vol. 794. 2010, p. 85. Dor: 10.1007/978-3-540-76937-8_4.

Gammie, C. F., J. C. McKinney, and G. Tóth. “HARM: A Numerical Scheme for General Relativistic Magnetohydrodynamics". In: ApJ 589 (May 2003), pp. 444-457. DoI: 10. 1086/374594. eprint: astro-ph/0301509.

Gammie, C. F., S. L. Shapiro, and J. C. McKinney. “Black Hole Spin Evolution”. In: ApJ 602 (Feb. 2004), pp. 312-319. DOI: 10. 1086/380996. eprint: astro-ph/0310886.

George, M. R. et al. “On active galactic nuclei as sources of ultra-high energy cosmic rays". In: MNRAS 388.1 (2008), pp. L59-L63. DOI: 10. 1111/ j . $1745-3933.2008$. Q0499.x. arXiv: 0805.2053 [astro-ph].

Ghisellini, G. and A. Celotti. “The dividing line between FR I and FR II radio-galaxies”. In: AEA 379 (2001), pp. L1-L4. DoI: 10.1051/0004-6361: 20011338. arXiv: astro$\mathrm{ph} / 0106570$ [astro-ph].

Ghisellini, G., L. Maraschi, and F. Tavecchio. “The Fermi blazars' divide”. In: MNRAS 396 (June 2009), pp. L105-L109. Dor: 10.1111/ j . 1745-3933.2009 .00673 . x. arXiv: 0903.2043 [astro-ph. CO].

Ghisellini, G. and F. Tavecchio. "Canonical high-power blazars". In: MNRAS 397.2 (2009), pp. 985-1002. Dor: 10.1111/j.1365-2966.2009.15007 x. arXiv: 0902.0793 [astro-ph.c0].

Ghisellini, G. and F. Tavecchio. “Fermi/LAT broad emission line blazars”. In: Monthly Notices of the Royal Astronomical Society 448.2 (Feb. 2015), pp. 1060-1077. IssN: 00358711. DoI: 10. 1093 /mnras / stv055. eprint: http : / / oup . prod. sis. lan/mnras / article-pdf/448/2/1060/13767377/stv055 . pdf. uRL: https: / / doi . org/10. 1093/mnras/stv055. 
Ghisellini, G. and F. Tavecchio. "The blazar sequence: a new perspective". In: MNRAS 387.4 (2008), pp. 1669-1680. Dor: 10.1111/ j. 1365-2966.2008.13360. x. arXiv: Q802.1918 [astro-ph].

Ghisellini, G. et al. "General physical properties of bright Fermi blazars". In: MNRAS 402 (Feb. 2010), pp. 497-518. DoI: 10.1111/ j . 1365-2966.2009 . 15898 . x. arXiv: 0909.0932 [astro-ph.CO].

Ghisellini, G. et al. “The Fermi blazar sequence”. In: MNRAS 469.1 (2017), pp. 255-266. DOI: $10.1093 / \mathrm{mnras} / \mathrm{stx} 806$. arXiv: 1702.02571 [astro-ph.HE].

Ghisellini, G. et al. "The power of relativistic jets is larger than the luminosity of their accretion disks". In: Nature 515 (Nov. 2014), pp.376-378. DoI: 10. 1038/nature13856. arXiv: 1411.5368 [astro-ph.HE].

Giacconi, R. et al. "Evidence for x Rays From Sources Outside the Solar System". In: Phys. Rev. Lett. 9.11 (1962), pp. 439-443. Dor: 10.1103/PhysRevLett. 9. 439.

Giannios, D. "Reconnection-driven plasmoids in blazars: fast flares on a slow envelope”. In: MNRAS 431.1 (2013), pp.355-363. DoI: 10. 1093/mnras/stt167. arXiv: 1211.0296 [astro-ph.HE].

Giannios, D., D. A. Uzdensky, and M. C. Begelman. “Fast TeV variability in blazars: jets in a jet". In: MNRAS 395.1 (2009), pp. L29-L33. Dor: 10.1111/j . 1745-3933 . 2009. Q0635.x. arXiv: 0901.1877 [astro-ph.HE].

Hardcastle, M. J. et al. "Radio and X-Ray Observations of the Jet in Centaurus A". In: ApJ 593.1 (2003), pp. 169-183. Dor: 10.1086/376519. arXiv: astro-ph/0304443 [astro-ph].

Harten, A., P. D. Lax, and B. v. Leer. “On Upstream Differencing and Godunov-Type Schemes for Hyperbolic Conservation Laws". In: SIAM Review 25.1 (1983), pp. 3561. DOI: 10.1137 / 1025002. eprint: https : / / doi . org / 10.1137 / 1025002. URL: https: //doi.org/10.1137/1025002. 
Hawking, S. W. “Black hole explosions?" In: Nature 248 (Mar. 1974), pp. 30-31. DoI: 10.1038/248030a0.

Hughes, M. P. “Radio Brightness Contours of 3C273”. In: Nature 207.4993 (1965), pp. 178-179. DOI: $10.1038 / 207178$ a0.

Inoue, Y. et al. "Disk-Jet Connection in Active Supermassive Black Holes in the Standard Accretion Disk Regime”. In: ApJ 840.1, 46 (2017), p. 46. Dor: 10. 3847/1538-4357/ aa6b57. arXiv: 1704.00123 [astro-ph.HE].

Jester, S. "A Simple Test for the Existence of Two Accretion Modes in Active Galactic Nuclei". In: ApJ 625 (June 2005), pp. 667-679. Dor: 10.1086/429812. eprint: astro$\mathrm{ph} / 0502394$.

Kadowaki, L. H. S., E. M. De Gouveia Dal Pino, and J. M. Stone. “MHD Instabilities in Accretion Disks and Their Implications in Driving Fast Magnetic Reconnection". In: ApJ 864.1, 52 (2018), p. 52. Dor: 10. 3847/1538-4357/aad4ff. arXiv: 1803.08557 [astro-ph.HE].

Kagan, D. et al. "Relativistic Magnetic Reconnection in Pair Plasmas and Its Astrophysical Applications". In: Space Sci. Rev. 191.1-4 (Oct. 2015), pp. 545-573. DoI: 10.1007/s11214-014-0132-9. arXiv: 1412.2451 [astro-ph.HE].

Kellermann, K. I. et al. "Sub-Milliarcsecond Imaging of Quasars and Active Galactic Nuclei. III. Kinematics of Parsec-scale Radio Jets". In: ApJ 609.2 (2004), pp. 539-563. DOI: $10.1086 / 421289$. arXiv: astro-ph/0403320 [astro-ph].

Kerr, R. P. “Gravitational Field of a Spinning Mass as an Example of Algebraically Special Metrics". In: Phys. Rev. Lett. 11 (5 1963), pp. 237-238. Dor: 10.1103/PhysRevLett. 11.237. uRL: https://link.aps.org/doi/10.1103/PhysRevLett.11.237.

Khiali, B. and E. M. de Gouveia Dal Pino. "High-energy neutrino emission from the core of low luminosity AGNs triggered by magnetic reconnection acceleration". In: MNRAS 455.1 (2016), pp. 838-845. DoI: 10.1093/mnras/stv2337. arXiv: 1506.01063 [astro-ph.HE]. 
Khiali, B., E. M. de Gouveia Dal Pino, and M. V. del Valle. "A magnetic reconnection model for explaining the multiwavelength emission of the microquasars Cyg X-1 and Cyg X-3". In: MNRAS 449 (May 2015), pp. 34-48. Dor: 10. 1093/mnras/stv248. arXiv: 1406.5664 [astro-ph.HE].

King, A. R., J. E. Pringle, and J. A. Hofmann. “The evolution of black hole mass and spin in active galactic nuclei". In: MNRAS 385 (Apr. 2008), pp. 1621-1627. DoI: 10.1111/j.1365-2966.2008.12943.x. arXiv: 0801.1564.

Komissarov, S. S. "A Godunov-type scheme for relativistic magnetohydrodynamics". In: MNRAS 303.2 (1999), pp. 343-366. DoI: 10.1046/j . 1365-8711.1999.02244 . x.

Kormendy, J. and L. C. Ho. “Coevolution (Or Not) of Supermassive Black Holes and Host Galaxies". In: ARAEA 51 (Aug. 2013), pp. 511-653. Dor: 10.1146/annurevastro-082708-101811. arXiv: 1304.7762 [astro-ph.CO].

Kowal, G., E. M. de Gouveia Dal Pino, and A. Lazarian. “Magnetohydrodynamic Simulations of Reconnection and Particle Acceleration: Three-dimensional Effects". In: ApJ 735, 102 (July 2011), p. 102. DOI: 10. 1088 /0004-637X / 735 / / / 102. arXiv: 1103.2984 [astro-ph.HE].

Landau, L. D. and E. M. Lifshitz. Fluid Mechanics. 2nd. Vol. 6. Course of Theoretical Physics. Butterworth-Heinemann, 1987. IsBN: 978-0-7506-2767-2.

Leahy, J. P. and R. A. Perley. “VLA Images of 23 Extragalactic Radio Sources”. In: AJ 102 (1991), p. 537. DOI: 10.1086/115892.

León-Tavares, J. et al. “EXPLORING THE RELATION BETWEEN (SUB-)MILLIMETER RADIATION AND $\gamma$-RAY EMISSION IN BLAZARS WITHPLANCKANDFERMI". In: The Astrophysical Journal 754.1 (2012), p. 23. Dor: 10.1088/0004-637x/754/1/23. URL: https: //doi .org/10. 1088\%2F0004-637x\%2F754\%2F1\%2F23.

Lewis, T. R., J. D. Finke, and P. A. Becker. “A Steady-state Spectral Model for Electron Acceleration and Cooling in Blazar Jets: Application to 3C 279". In: ApJ 853.1, 6 (2018), p. 6. DOI: 10.3847/1538-4357/aaa19a. arXiv: 1710.01334 [astro-ph.GA]. 
Lewis, T. R., J. D. Finke, and P. A. Becker. “Electron Acceleration in Blazars: Application to the 3C 279 Flare on 2013 December 20". In: ApJ 884.2, 116 (2019), p. 116. DoI: 10. 3847/1538-4357/ab43c3. arXiv: 1909.04431 [astro-ph.GA].

Li, X. et al. “Nonthermally Dominated Electron Acceleration during Magnetic Reconnection in a Low- Plasma". In: ApJL 811.2 (2015), p. L24. uRL: http: //stacks . iop. org $/ 2041-8205 / 811 / i=2 / a=L 24$.

Lichnerowicz, A. Relativistic Hydrodynamics and Magnetohydrodynamics. 1st. The Mathematical Physics Monograph Series. W. A. Benjamin, Inc., 1967.

Liska, M. et al. “Bardeen-Petterson alignment, jets, and magnetic truncation in GRMHD simulations of tilted thin accretion discs". In: MNRAS 487.1 (2019), pp. 550-561. DoI: 10.1093/mnras/stz834. arXiv: 1810.00883 [astro-ph.HE].

Liska, M. T. P., A. Tchekhovskoy, and E. Quataert. “Large-Scale Poloidal Magnetic Field Dynamo Leads to Powerful Jets in GRMHD Simulations of Black Hole Accretion with Toroidal Field". In: arXiv e-prints, arXiv:1809.04608 (2018), arXiv:1809.04608. arXiv: 1809.04608 [astro-ph.HE].

Lobanov, A. P. “Ultracompact jets in active galactic nuclei”. In: AEA 330 (Feb. 1998), pp. 79-89. eprint: astro-ph/9712132.

Madejski, G. G. and M. Sikora. “Gamma-Ray Observations of Active Galactic Nuclei”. In: ARAEA 54 (2016), pp. 725-760. DoI: 10.1146/annurev-astro-081913-040044.

Marrone, D. P. et al. "An Unambiguous Detection of Faraday Rotation in Sagittarius A*”. In: ApJL 654.1 (2007), pp. L57-L60. Dor: 10. 1086/510850. arXiv: astro-ph/0611791 [astro-ph].

Marshall, H. L. et al. "Structure of the X-Ray Emission from the Jet of 3C 273". In: ApJL 549.2 (2001), pp. L167-L171. DOI: 10. 1086/319161. arXiv: astro-ph/0012162 [astro-ph]. 
Massaro, F., D. J. Thompson, and E. C. Ferrara. “The extragalactic gamma-ray sky in the Fermi era". In: AEAR 24, 2 (Dec. 2016), p. 2. DoI: 10.1007/s00159-015-0090-6. arXiv: 1510.07660 [astro-ph.HE].

Massaro, F. et al. “UNVEILING THE NATURE OF THE UNIDENTIFIED GAMMA-RAY SOURCES. III. GAMMA-RAY BLAZAR-LIKE COUNTERPARTS AT LOW RADIO FREQUENCIES". In: The Astrophysical Journal Supplement Series 207.1 (2013), p. 4. DOI: 10 . 1088 /0067-0049/207 / 1 / 4. URL: https : / / doi . org / 10 . 1088\%2F0067QQ49\%2F 207\%2F 1\%2F 4.

Mastichiadis, A. and J. G. Kirk. "Self-consistent particle acceleration in active galactic nuclei." In: AEA 295 (1995), p. 613.

McClintock, J. E. et al. "The Spin of the Near-Extreme Kerr Black Hole GRS 1915+105". In: ApJ 652.1 (2006), pp. 518-539. Dor: 10.1086/508457. arXiv: astro-ph/0606076 [astro-ph].

McKinney, J. C. and C. F. Gammie. "A Measurement of the Electromagnetic Luminosity of a Kerr Black Hole”. In: ApJ 611 (Aug. 2004), pp. 977-995. Dor: 10. 1086/422244. eprint: arXiv: astro-ph/0404512.

McKinney, J. C., A. Tchekhovskoy, and R. D. Blandford. "General relativistic magnetohydrodynamic simulations of magnetically choked accretion flows around black holes". In: MNRAS 423 (July 2012), pp. 3083-3117. Dor: 10. 1111/j . 1365-2966. 2012.21074.x. arXiv: 1201.4163 [astro-ph.HE].

McKinney, J. C. et al. “Three-dimensional general relativistic radiation magnetohydrodynamical simulation of super-Eddington accretion, using a new code HARMRAD with M1 closure". In: MNRAS 441 (July 2014), pp. 3177-3208. Dor: 10. 1093/mnras/ stu762. arXiv: 1312.6127.

McLure, R. J. and J. S. Dunlop. “The cosmological evolution of quasar black hole masses". In: MNRAS 352.4 (Aug. 2004), pp. 1390-1404. Dor: 10.1111 / j . 1365 2966.2004.08034.x. arXiv: astro-ph/0310267 [astro-ph]. 
Meier, D. L. “The Association of Jet Production with Geometrically Thick Accretion Flows and Black Hole Rotation”. In: ApJL 548 (Feb. 2001), pp. L9-L12. Dor: 10 . 1086/ 318921. eprint: arXiv:astro-ph/0010231.

Meisenheimer, K. et al. “The synchrotron spectra of radio hot spots.” In: $A \mathcal{E} A 219$ (1989), pp. 63-86.

Meyer, E. T. et al. "From the Blazar Sequence to the Blazar Envelope: Revisiting the Relativistic Jet Dichotomy in Radio-loud Active Galactic Nuclei". In: ApJ 740, 98 (Oct. 2011), p. 98. DOI: 10.1088/0004-637X/740/2/98. arXiv: 1107.5105 [astro-ph. C0].

Migliari, S. and R. P. Fender. "Jets in neutron star X-ray binaries: a comparison with black holes". In: MNRAS 366.1 (2006), pp. 79-91. Dor: 10. 1111/j . 1365-2966. 2005. 09777 . x. arXiv: astro-ph/0510698 [astro-ph].

Miller, M. C. and E. J. M. Colbert. “Intermediate-Mass Black Holes”. In: International Journal of Modern Physics D 13.1 (2004), pp. 1-64. DoI: 10. 1142/S0218271804004426. arXiv: astro-ph/0308402 [astro-ph].

Misner, C. W., K. S. Thorne, and J. A. Wheeler. Gravitation. W. H. Freeman and Company, 1973.

Moffet, A. T. "The Structure of Radio Galaxies". In: ARAEA 4 (1966), p. 145. DoI: 10.1146/annurev.aa.04.090166.001045.

Mościbrodzka, M. and H. Falcke. “Coupled jet-disk model for Sagittarius A*: explaining the flat-spectrum radio core with GRMHD simulations of jets". In: $A \mathcal{E} A$ 559, L3 (Nov. 2013), p. L3. DOI: 10. 1051/0004-6361/201322692. arXiv: 1310.4951 [astro-ph. HE].

Mościbrodzka, M., H. Falcke, and H. Shiokawa. “General relativistic magnetohydrodynamical simulations of the jet in M 87". In: AESA 586, A38 (Feb. 2016), A38. DoI: 10.1051/0004-6361/201526630. arXiv: 1510.07243 [astro-ph.HE].

Mościbrodzka, M. and C. F. Gammie. “IPOLE - semi-analytic scheme for relativistic polarized radiative transport". In: MNRAS 475 (Mar. 2018), pp. 43-54. Dor: 10 . 1093/mnras/stx3162. arXiv: 1712.03057 [astro-ph.HE]. 
Mościbrodzka, M. et al. "Faraday rotation in GRMHD simulations of the jet launching zone of M87". In: MNRAS 468 (June 2017), pp. 2214-2221. Dor: 10.1093 /mnras / stx587. arXiv: 1703.02390 [astro-ph.HE].

Mościbrodzka, M. et al. “Observational appearance of inefficient accretion flows and jets in 3D GRMHD simulations: Application to Sagittarius A*". In: AEA 570, A7 (Oct. 2014), A7. DOI: 10.1051/0004-6361/201424358. arXiv: 1408.4743 [astro-ph.HE].

Mościbrodzka, M. et al. "Radiative Models of SGR A* from GRMHD Simulations". In: ApJ 706 (Nov. 2009), pp. 497-507. DoI: 10 . 1088/0004-637X/706/1/497. arXiv: Q909.5431 [astro-ph.HE].

Narayan, R., I. V. Igumenshchev, and M. A. Abramowicz. “Magnetically Arrested Disk: an Energetically Efficient Accretion Flow". In: PASJ 55 (Dec. 2003), pp. L69-L72. eprint: arXiv: astro-ph/0305029.

Narayan, R., R. Mahadevan, and E. Quataert. “Advection-dominated accretion around black holess". In: Theory of Black Hole Accretion Disks. Ed. by M. A. Abramowicz, G. Bjornsson, and J. E. Pringle. 1998, p. 148. eprint: arXiv: astro-ph/9803141.

Narayan, R. and I. Yi. “Advection-dominated accretion: A self-similar solution”. In: ApJL 428 (June 1994), pp. L13-L16. DoI: 10. 1086/187381. eprint: astro-ph/9403052.

— "Advection-dominated Accretion: Underfed Black Holes and Neutron Stars". In: ApJ 452 (Oct. 1995), p. 710. DoI: 10. 1086/176343. eprint: arXiv : astro-ph/9411059.

Narayan, R., I. Yi, and R. Mahadevan. "Explaining the spectrum of Sagittarius A* with a model of an accreting black hole". In: Nature 374 (Apr. 1995), pp. 623-625. DoI: $10.1038 / 374623 \mathrm{a} 0$.

Nemmen, R. S. et al. "A Universal Scaling for the Energetics of Relativistic Jets from Black Hole Systems”. In: Science 338 (Dec. 2012), pp. 1445-. Dor: 10. 1126/science. 1227416. arXiv: 1212.3343 [astro-ph.HE]. 
Nemmen, R. S. et al. "Models for jet power in elliptical galaxies: a case for rapidly spinning black holes". In: MNRAS 377 (June 2007), pp. 1652-1662. Dor: 10.1111/j . 1365-2966.2007.11726.x. eprint: arXiv: astro-ph/0612354.

Netzer, H. "Revisiting the Unified Model of Active Galactic Nuclei". In: ARAEA 53 (Aug. 2015), pp. 365-408. DoI: 10.1146/annurev-astro-082214-122302. arXiv: 1505.00811.

Noble, S. C., J. H. Krolik, and J. F. Hawley. “Direct Calculation of the Radiative Efficiency of an Accretion Disk Around a Black Hole". In: ApJ 692 (Feb. 2009), pp. 411-421. DOI: $10.1088 / 0004-637 \mathrm{X} / 692 / 1 / 411$. arXiv: 0808.3140.

Noble, S. C., J. H. Krolik, and J. F. Hawley. “Dependence of Inner Accretion Disk Stress on Parameters: The Schwarzschild Case". In: ApJ 711.2 (2010), pp. 959-973. DoI: 10.1088/0004-637X/711/2/959. arXiv: 1001.4809 [astro-ph.HE].

Noble, S. C. et al. "Primitive Variable Solvers for Conservative General Relativistic Magnetohydrodynamics". In: ApJ 641.1 (2006), pp. 626-637. DoI: 10. 1086/500349. arXiv: astro-ph/0512420 [astro-ph].

Noble, S. C. et al. "Radiative Efficiency and Thermal Spectrum of Accretion onto Schwarzschild Black Holes". In: ApJ 743.2, 115 (2011), p. 115. Dor: 10 . 1088/0004637X/743/2/115. arXiv: 1105.2825 [astro-ph.HE].

Novikov, I. D. and K. S. Thorne. “Astrophysics of black holes.” In: Black Holes (Les Astres Occlus). Ed. by C. Dewitt and B. S. Dewitt. 1973, pp. 343-450.

Oppenheimer, J. R. and G. M. Volkoff. “On Massive Neutron Cores”. In: Phys. Rev. 55 (4 1939), pp. 374-381. DoI: 10.1103/PhysRev . 55 . 374. uRL: https : //link . aps . org/ doi/10.1103/PhysRev. 55.374.

Padovani, P. et al. "Active galactic nuclei: what's in a name?" In: AEAR 25.1, 2 (2017), p. 2. DOI: 10.1007 /s00159-017-0102-9. arXiv: 1707.07134 [astro-ph.GA].

Paliya, V. S. “FERMI -LARGE AREA TELESCOPE OBSERVATIONS OF THE EXCEPTIONAL GAMMA-RAY FLARE FROM 3C 279 IN 2015 JUNE". In: The Astrophysical 
Journal 808.2 (2015), p. L48. DOI: 10.1088 / $2041-8205$ / 808/2/148. URL: https: //doi.org/10.1088\%2F2041-8205\%2F808\%2F2\%2F148.

Paliya, V. S. et al. "General Physical Properties of CGRaBS Blazars”. In: ApJ 851.1, 33 (2017), p. 33. DoI: 10.3847/1538-4357/aa98e1. arXiv: 1711.01292 [astro-ph.HE].

Pandya, A. et al. "Polarized Synchrotron Emissivities and Absorptivities for Relativistic Thermal, Power-law, and Kappa Distribution Functions". In: ApJ 822, 34 (May 2016), p. 34. DOI: $10.3847 / 0004-637 \mathrm{X} / 822 / 1 / 34$. arXiv: 1602.08749 [astro-ph.HE].

Parfrey, K., A. Philippov, and B. Cerutti. “First-Principles Plasma Simulations of BlackHole Jet Launching". In: Phys. Rev. Lett. 122.3, 035101 (2019), p. 035101. Dor: 10. 1103/PhysRevLett. 122.035101 . arXiv: 1810.03613 [astro-ph.HE].

Penna, R. F. et al. "Simulations of magnetized discs around black holes: effects of black hole spin, disc thickness and magnetic field geometry". In: MNRAS 408.2 (2010), pp. 752-782. DoI: 10.1111/j.1365-2966.2010.17170.x. arXiv: 1003.0966 [astro-ph.HE].

Penrose, R. “Gravitational Collapse: the Role of General Relativity". In: Nuovo Cimento Rivista Serie 1 (1969), p. 252.

Perlman, E. S. et al. “The Deep X-Ray Radio Blazar Survey. I. Methods and First Results”. In: AJ 115.4 (1998), pp. 1253-1294. Dor: 10. 1086/300283. arXiv: astro-ph/9801024 [astro-ph].

Petropoulou, M. et al. "Plasmoid statistics in relativistic magnetic reconnection". In: MNRAS 475.3 (2018), pp. 3797-3812. DOI: 10.1093 /mnras / sty033. arXiv: 1710. Q0724 [astro-ph.HE].

Petropoulou, M., D. Giannios, and L. Sironi. “Blazar flares powered by plasmoids in relativistic reconnection". In: MNRAS 462 (Nov. 2016), pp. 3325-3343. Dor: 10. 1093/ mnras/stw1832. 
Pierre Auger Collaboration et al. "Correlation of the Highest-Energy Cosmic Rays with Nearby Extragalactic Objects". In: Science 318.5852 (2007), p. 938. Dor: 10 . 1126 / science.1151124. arXiv: 0711.2256 [astro-ph].

Pierre Auger Collaboration et al. "Correlation of the highest-energy cosmic rays with the positions of nearby active galactic nuclei". In: Astroparticle Physics 29.3 (2008), pp. 188-204. DoI: $10.1016 / \mathrm{j}$. astropartphys . 2008.01.002. arXiv: 0712.2843 [astro-ph].

Pjanka, P., A. A. Zdziarski, and M. Sikora. "The power and production efficiency of blazar jets". In: MNRAS 465.3 (2017), pp. 3506-3514. DoI: 10. 1093/mnras/stw2960. arXiv: 1607.08895 [astro-ph.HE].

Planck Collaboration et al. "Planck 2015 results. XIII. Cosmological parameters". In: AEA 594, A13 (Sept. 2016), A13. DoI: 10.1051/0004-6361/201525830. arXiv: 1502.01589.

Plotkin, R. M. et al. “Dynamical black hole masses of BL Lac objects from the Sloan Digital Sky Survey". In: MNRAS 413.2 (2011), pp. 805-812. Dor: 10 . 1111/ j . 1365 2966.2010.18172.x. arXiv: 1012.1601 [astro-ph.C0].

Porth, O. et al. "The Event Horizon General Relativistic Magnetohydrodynamic Code Comparison Project". In: ApJS 243.2, 26 (2019), p. 26. Dor: 10 . 3847 / 1538-4365 / ab29fd. arXiv: 1904.04923 [astro-ph.HE].

Rees, M. J. “Appearance of Relativistically Expanding Radio Sources”. In: Nature 211 (July 1966), pp. 468-470. Dor: 10.1038/211468a0.

_ "Black Hole Models for Active Galactic Nuclei". In: ARAEA 22 (1984), pp. 471-506. DoI: 10.1146/annurev . aa.22.090184.002351.

Ressler, S. M. et al. “Electron thermodynamics in GRMHD simulations of low-luminosity black hole accretion". In: MNRAS 454 (Dec. 2015), pp. 1848-1870. Dor: 10. 1093 / mnras/stv2084. arXiv: 1509.04717 [astro-ph.HE]. 
Ressler, S. M. et al. “The disc-jet symbiosis emerges: modelling the emission of Sagittarius A* with electron thermodynamics". In: MNRAS 467 (May 2017), pp. 3604-3619. DOI: 10.1093/mnras/stx364. arXiv: 1611.09365 [astro-ph.HE].

Rusinek, K. et al. "On the efficiency of jet production in FR II radio galaxies and quasars". In: Monthly Notices of the Royal Astronomical Society 466.2 (Dec. 2016), pp. 2294-2301. ISSN: 0035-8711. DOI: 10.1093/mnras / stw3330. eprint: http : / / oup . prod. sis . lan /mnras / article - pdf / 466 / 2 / 2294 / 10868600 / stw3330 . pdf. URL: https: //doi . org/10.1093/mnras/stw3330.

Ryan, B. R., J. C. Dolence, and C. F. Gammie. “bhlight: General Relativistic Radiation Magnetohydrodynamics with Monte Carlo Transport”. In: ApJ 807, 31 (July 2015), p. 31. DOI: 10.1088/0004-637X/807/1/31. arXiv: 1505.05119 [astro-ph.HE].

Ryan, B. R. et al. "The Radiative Efficiency and Spectra of Slowly Accreting Black Holes from Two-temperature GRRMHD Simulations". In: ApJL 844, L24 (Aug. 2017), p. L24. DOI: 10.3847/2041-8213/aa8034. arXiv: 1707.04238 [astro-ph.HE].

Sambruna, R. M. et al. "SWIFTBURST ALERT TELESCOPE,FERMILARGE AREA TELESCOPE, AND THE BLAZAR SEQUENCE". In: The Astrophysical Journal 710.1 (2010), pp. 24-28. DOI: 10. 1088/0004-637x/710/1/24. uRL: https: //doi .org/10. 1088\%2F0004-637x\%2F710\%2F 1\%2F 24.

Sa̧dowski, A. et al. "Energy, momentum and mass outflows and feedback from thick accretion discs around rotating black holes". In: MNRAS 436 (Dec. 2013), pp. 38563874. DoI: 10.1093/mnras/stt1881. arXiv: 1307.1143 [astro-ph.HE].

Sa̧dowski, A. et al. "Numerical simulations of super-critical black hole accretion flows in general relativity". In: MNRAS 439 (Mar. 2014), pp. 503-520. Dor: 10. 1093/mnras/ stt2479. arXiv: 1311.5900 [astro-ph.HE].

Sasdowski, A. et al. "Spinning up black holes with super-critical accretion flows". In: AEA 532, A41 (Aug. 2011), A41. DoI: 10. $1051 / 0004-6361 / 201116702$. arXiv: 1102.2456 [astro-ph.HE]. 
Schmidt, M. “3C 273 : A Star-Like Object with Large Red-Shift”. In: Nature 197.4872 (1963), p. 1040. DoI: 10.1038/1971040a0.

Schreier, E. J., P. Gorenstein, and E. D. Feigelson. "High-resolution X-ray observations of M87 - Nucleus, jet and radio halo". In: ApJ 261 (1982), pp. 42-50. Dor: 10. 1086/ 160316.

Semenov, V., S. Dyadechkin, and B. Punsly. "Simulations of Jets Driven by Black Hole Rotation”. In: Science 305 (Aug. 2004), pp. 978-980. Dor: 10.1126/science. 1100638. eprint: astro-ph/0408371.

Shabala, S. S., J. S. Santoso, and L. E. H. Godfrey. “MEASURING THE JET POWER OF FLAT-SPECTRUM RADIO QUASARS". In: The Astrophysical Journal 756.2 (2012), p. 161. DOI: $10.1088 / 0004-637 x / 756 / 2 / 161$. uRL: https : / / doi . org/10 . 1088\% 2F0004-637x\%2F756\%2F2\%2F 161.

Shafee, R., R. Narayan, and J. E. McClintock. "Viscous Torque and Dissipation in the Inner Regions of a Thin Accretion Disk: Implications for Measuring Black Hole Spin”. In: ApJ 676.1 (2008), pp. 549-561. Dor: 10. 1086/527346. arXiv: 0705.2244 [astro-ph].

Shafee, R. et al. "Estimating the Spin of Stellar-Mass Black Holes by Spectral Fitting of the X-Ray Continuum". In: ApJL 636.2 (2006), pp. L113-L116. Dor: 10 . 1086/498938. arXiv: astro-ph/0508302 [astro-ph].

Shafee, R. et al. "Three-Dimensional Simulations of Magnetized Thin Accretion Disks around Black Holes: Stress in the Plunging Region". In: ApJL 687.1 (2008), p. L25. DOI: $10.1086 / 593148$. arXiv: 0808.2860 [astro-ph].

Shakura, N. I. and R. A. Sunyaev. "Black holes in binary systems. Observational appearance." In: AEA 24 (1973), pp. 337-355.

Shaw, M. S. et al. "Spectroscopy of the Largest Ever $\gamma$-Ray-selected BL Lac Sample". In: ApJ 764, 135 (Feb. 2013), p. 135. DOI: 10. 1088/0004-637X/764/2/135. arXiv: 1301.0323 [astro-ph.HE]. 
Shaw, M. S. et al. "SPECTROSCOPY OF BROAD-LINE BLAZARS FROM 1LAC". In: The Astrophysical Journal 748.1 (2012), p. 49. Dor: 10.1088/0004-637x/748/1/49. URL: https: //doi . org/10 . 1088\%2F0004-637x\%2F748\%2F1\%2F49.

Sikora, M., Ł. Stawarz, and J.-P. Lasota. “Radio Loudness of Active Galactic Nuclei: Observational Facts and Theoretical Implications". In: ApJ 658 (Apr. 2007), pp. 815828. DoI: 10.1086/511972. eprint: arXiv: astro-ph/0604095.

Sironi, L., D. Giannios, and M. Petropoulou. "Plasmoids in relativistic reconnection, from birth to adulthood: first they grow, then they go". In: MNRAS 462 (Oct. 2016), pp. 48-74. DOI: 10.1093/mnras/stw1620.

Sironi, L., M. Petropoulou, and D. Giannios. "Relativistic jets shine through shocks or magnetic reconnection?" In: MNRAS 450 (June 2015), pp. 183-191. Dor: 10 . 1093 / mnras/stv641.

Sironi, L. and A. Spitkovsky. "Relativistic Reconnection: An Efficient Source of Nonthermal Particles". In: ApJL 783.1, L21 (2014), p. L21. Dor: 10 . 1088/2041-8205/783/ 1/L21. arXiv: 1401.5471 [astro-ph.HE].

Su, M., T. R. Slatyer, and D. P. Finkbeiner. "Giant Gamma-ray Bubbles from FermiLAT: Active Galactic Nucleus Activity or Bipolar Galactic Wind?" In: ApJ 724 (Dec. 2010), pp. 1044-1082. DoI: 10.1088/0004-637X/724/2/1044. arXiv: 1005.5480 [astro-ph.HE].

Tadhunter, C. "An introduction to active galactic nuclei: Classification and unification". In: 52 (Aug. 2008), pp. 227-239. DoI: 10.1016/j . newar. 2008.06.004.

Tadhunter, C. "Radio AGN in the local universe: unification, triggering and evolution”. In: $A \mathcal{E} A R$ 24.1, 10 (2016), p. 10. DoI: 10.1007/s00159-016-0094-x. arXiv: 1605 . 08773 [astro-ph.GA].

Tchekhovskoy, A., R. Narayan, and J. C. McKinney. “Black Hole Spin and The Radio Loud/Quiet Dichotomy of Active Galactic Nuclei". In: ApJ 711 (Mar. 2010), pp. 5063. DoI: $10.1088 / 0004-637 \mathrm{X} / 711 / 1 / 50$. arXiv: 0911.2228 [astro-ph.HE]. 
Tchekhovskoy, A., R. Narayan, and J. C. McKinney. “Efficient generation of jets from magnetically arrested accretion on a rapidly spinning black hole". In: MNRAS 418 (Nov. 2011), pp. L79-L83. DoI: 10.1111/ j . 1745 - 3933.2011 .01147 . x. arXiv: 1108.0412 [astro-ph.HE].

The EHT Collaboration. "First M87 Event Horizon Telescope Results. I. The Shadow of the Supermassive Black Hole". In: ApJL 875 (2019), p. 1. uRL: https: //iopscience. iop.org/article/10.3847/2041-8213/ab0ec7.

The Fermi-LAT Collaboration. “Fermi Large Area Telescope Fourth Source Catalog”. In: (Feb. 26, 2019). arXiv: 1902 . 10045 [astro-ph.HE].

Thorne, K. S. “Disk-Accretion onto a Black Hole. II. Evolution of the Hole". In: ApJ 191 (July 1974), pp. 507-520. DoI: 10. 1086/152991.

Tolman, R. C. "Static Solutions of Einstein's Field Equations for Spheres of Fluid". In: Phys. Rev. 55 (4 1939), pp. 364-373. DoI: 10.1103/PhysRev . 55 . 364. uRL: https: //link.aps.org/doi/10.1103/PhysRev . 55.364.

Toro, E. F. Riemann Solvers and Numerical Methods in Fluid Dynamics: A Practical Introduction. 2nd. Berlin: Springer, 1999. ISBN: 3-540-65966-8.

Urry, C. M. and P. Padovani. “Unified Schemes for Radio-Loud Active Galactic Nuclei”. In: PASP 107 (Sept. 1995), p. 803. Dor: 10.1086/133630. eprint: astro-ph/9506063. van Velzen, S. and H. Falcke. "The contribution of spin to jet-disk coupling in black holes". In: AEA 557, L7 (Sept. 2013), p. L7. DoI: 10 . 1051/0004-6361/201322127. arXiv: 1308.1437 [astro-ph.HE].

Venemans, B. P. et al. “Copious Amounts of Dust and Gas in a z = 7.5 Quasar Host Galaxy". In: ApJL 851.1, L8 (2017), p. L8. Dor: 10 . 3847 / 2041 - 8213/aa943a. arXiv: 1712.01886 [astro-ph.GA].

Volonteri, M. “The Formation and Evolution of Massive Black Holes". In: Science 337 (Aug. 2012), pp. 544-. DoI: 10.1126/science. 1220843. arXiv: 1208.1106 [astro-ph. CO]. 
Volonteri, M., M. Sikora, and J.-P. Lasota. "Black Hole Spin and Galactic Morphology". In: ApJ 667 (Oct. 2007), pp. 704-713. Dor: 10.1086/521186. arXiv: 0706.3900.

Volonteri, M. et al. "The Evolution of Active Galactic Nuclei and their Spins". In: ApJ 775, 94 (Oct. 2013), p. 94. DoI: 10.1088/0004-637X/775/2/94. arXiv: 1210.1025 [astro-ph.HE].

Volonteri, M. et al. “The Distribution and Cosmic Evolution of Massive Black Hole Spins". In: ApJ 620.1 (2005), pp. 69-77. DoI: 10.1086/426858. arXiv: astro-ph / Q410342 [astro-ph].

Walker, R. C. et al. “The Structure and Dynamics of the Subparsec Jet in M87 Based on 50 VLBA Observations over 17 Years at 43 GHz". In: ApJ 855.2, 128 (2018), p. 128. DOI: $10.3847 / 1538-4357 /$ aaafcc. arXiv: 1802.06166 [astro-ph.HE].

Werner, G. R. et al. “Non-thermal particle acceleration in collisionless relativistic electronproton reconnection". In: MNRAS 473.4 (2018), pp. 4840-4861. Dor: 10. 1093/mnras / stx2530. arXiv: 1612.04493 [astro-ph.HE].

Werner, G. R., A. A. Philippov, and D. A. Uzdensky. “Particle acceleration in relativistic magnetic reconnection with strong inverse-Compton cooling in pair plasmas". In: MNRAS 482.1 (2019), pp. L60-L64. Dor: 10. 1093/mnrasl/sly157. arXiv: 1805 . 01910 [astro-ph.HE].

Werner, G. R. and D. A. Uzdensky. “Nonthermal Particle Acceleration in 3D Relativistic Magnetic Reconnection in Pair Plasma". In: ApJL 843.2, L27 (2017), p. L27. DOI: 10.3847/2041-8213/aa7892. arXiv: 1705.05507 [astro-ph.HE].

Willott, C. J. et al. "The emission line-radio correlation for radio sources using the 7C Redshift Survey". In: Monthly Notices of the Royal Astronomical Society 309.4 (Nov. 1999), pp. 1017-1033. ISSN: 0035-8711. DOI: 10.1046/ j . 1365-8711 . 1999. 02907 . x. eprint: http: //oup . prod. sis . lan/mnras/article-pdf/309/4/1017/3785912/ 309-4-1017.pdf. uRL: https: //doi . org/10.1046/j . 1365-8711 . 1999.02907 . x. 
Yuan, F. and R. Narayan. "Hot Accretion Flows Around Black Holes". In: ARAEA 52 (Aug. 2014), pp. 529-588. DoI: 10.1146/annurev-astro-082812-141003. arXiv: 1401.0586 [astro-ph.HE].

Yuan, F., E. Quataert, and R. Narayan. "Nonthermal Electrons in Radiatively Inefficient Accretion Flow Models of Sagittarius A*”. In: ApJ 598.1 (2003), pp. 301-312. DoI: 10.1086/378716. arXiv: astro-ph/0304125 [astro-ph].

Zamaninasab, $\mathrm{M}$ et al. “Dynamically important magnetic fields near accreting supermassive black holes". In: Nature 510.7503 (2014), pp. 126-128.

Zdziarski, A. A. et al. "Core shifts, magnetic fields and magnetization of extragalactic jets". In: MNRAS 451.1 (2015), pp. 927-935. DOI: 10 . 1093 /mnras / stv986. arXiv: 1410.7310 [astro-ph.HE].

Zenitani, S. and M. Hoshino. "The Generation of Nonthermal Particles in the Relativistic Magnetic Reconnection of Pair Plasmas". In: ApJL 562.1 (Nov. 2001), pp. L63-L66. DOI: 10.1086/337972. arXiv: 1402.7139 [astro-ph.HE].

Zhdankin, V. et al. "Statistical Analysis of Current Sheets in Three-dimensional Magnetohydrodynamic Turbulence". In: ApJ 771.2, 124 (2013), p. 124. Dor: 10. 1088/0004637X/771/2/124. arXiv: 1302.1460 [astro-ph.HE]. 